Supporting Information for

\title{
Macromolecular Ligand Engineering for Programmable Nanoprism Assembly
}

Yang Liu, ${ }^{1, \#}$ Marco Klement,, ${ }^{2, \#}$ Yi Wang, ${ }^{1, \#}$ Yaxu Zhong, ${ }^{1, \#}$ Baixu Zhu, ${ }^{1}$ Jun Chen, ${ }^{1}$ Michael Engel, 2,* Xingchen $\mathrm{Ye}^{1, *}$

${ }^{1}$ Department of Chemistry, Indiana University, Bloomington, Indiana 47405, USA.

${ }^{2}$ Institute for Multiscale Simulation, Interdisciplinary Center for Nanostructured Films, FriedrichAlexander University Erlangen-Nürnberg, 91058 Erlangen, Germany.

\# These authors contributed equally to this work.

${ }^{*}$ Correspondence should be addressed to M.E. (michael.engel@fau.de) and X.Y. (xingye@indiana.edu). 


\section{Section 1. Synthesis of pentaethylenehexamine-terminated polystyrene (PS-PEHA) ligands}

Chemicals. Styrene ( $\geq 99 \%$ ), ethyl $\alpha$-bromoisobutyrate (EBIB, 98\%), copper(II) bromide (99\%), tris[2-(dimethylamino)ethyl]amine (Me6TREN, 97\%), tin(II) 2-ethylhexanoate (Sn(EH)2, 92.5-100.0\%), pentaethylenehexamine (PEHA, technical grade), triethylamine ( $\mathrm{NEt}_{3}, \geq 99.5 \%$ ), $\mathrm{N}, \mathrm{N}$-dimethylformamide (DMF, anhydrous, 99.8\%), and toluene (anhydrous, 99.8\%) were purchased from Sigma Aldrich. Calcium hydride (90-95\%, $2 \mathrm{~mm}$ and down) was purchased from Alfa Aesar. Unless otherwise specified, reagents were used as received without further purification. Styrene was extracted with $10 \mathrm{wt} \%$ sodium hydroxide solution to remove inhibitors and distilled with $\mathrm{CaH}_{2}$ under vacuum.

Synthesis of PS-PEHA. PS-PEHA was synthesized by using a two-step reaction (Scheme S1). In the first step, bromine-terminated polystyrene (PS-Br) was synthesized by using activators regenerated by electron transfer atom transfer radical polymerization (ARGET ATRP). ${ }^{1}$ In the second step, the bromine end group was converted to pentaethylenehexamine, a multidentate nitrogen-containing ligand.

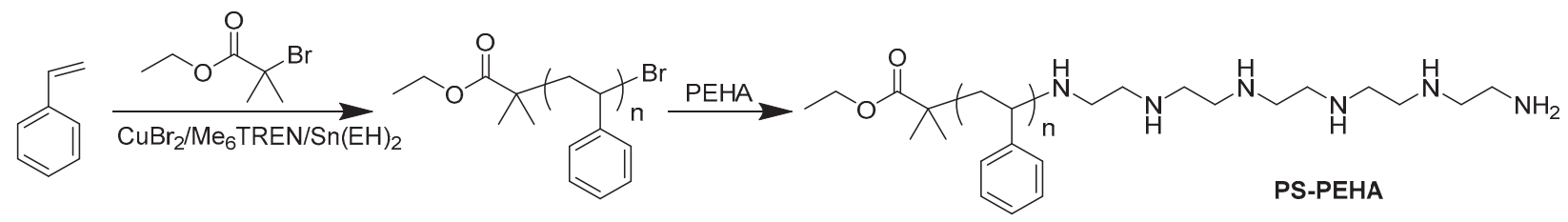

Scheme S1. Synthesis of PS-PEHA.

Step 1. Synthesis of PS-Br. In a typical reaction for PS-Br with molecular weight greater than 5 kDa, styrene (100-400 eq, 43.6 mmol), EBIB (1.0 eq), CuBr2 (0.01 eq) and Me6TREN (0.1 eq) were added to a round-bottom flask (Table S1). After purging with $\mathrm{N}_{2}$ for $30 \mathrm{~min}, \mathrm{Sn}(\mathrm{EH})_{2}(0.1$ eq) was injected and the reaction solution was heated to $90{ }^{\circ} \mathrm{C}$ under stirring. The reaction was terminated after 17-22 $\mathrm{h}$ by exposure to air while cooling the reaction flask with an ice bath.

In a typical reaction for PS-Br with molecular weight less than $5 \mathrm{kDa}$, styrene (20-50 eq, $43.6 \mathrm{mmol}$ ), anhydrous toluene, EBIB (1.0 eq), $\mathrm{CuBr}_{2}(0.01 \mathrm{eq})$ and Me6TREN (0.1 eq) were added to a round-bottom flask (Table S2). After purging with $\mathrm{N}_{2}$ for $30 \mathrm{~min}, \mathrm{Sn}(\mathrm{EH})_{2}(0.1 \mathrm{eq})$ was injected and the reaction solution was heated to $90{ }^{\circ} \mathrm{C}$ under stirring. The reaction was terminated after 14-20 $\mathrm{h}$ by exposure to air while cooling the reaction flask with an ice bath.

The reaction mixture was diluted with THF and precipitated using methanol. Afterwards, polystyrene was collected via vacuum filtration followed by re-dissolution in THF. To remove residual copper-based catalysts, the THF solution of polystyrene was mixed with neutral $\mathrm{Al}_{2} \mathrm{O}_{3}$ and stirred overnight followed by removing the $\mathrm{Al}_{2} \mathrm{O}_{3}$ powder using vacuum filtration. Polystyrene 
was precipitated again with methanol, vacuum filtered and dried inside a vacuum oven at $40{ }^{\circ} \mathrm{C}$ overnight.

Step 2. Conversion of the bromine end group to PEHA. PS-PEHA was synthesized according to the method recently reported by our group. ${ }^{2}$ In a typical reaction, $\mathrm{PS}-\mathrm{Br}$ (0.2 mmol), PEHA (10 $\mathrm{mmol}$ ) and triethylamine (20 mmol) were dissolved into DMF. The reaction mixture was stirred for $72 \mathrm{~h}$ at room temperature. Afterwards, PS-PEHA was precipitated with methanol and collected by vacuum filtration. PS-PEHA was purified three more times by dissolution in THF and precipitation in methanol before dried inside a vacuum oven at $40{ }^{\circ} \mathrm{C}$ overnight.

\section{Section 2. Nanocrystal synthesis and characterization}

Chemicals. $\mathrm{Gd}_{2} \mathrm{O}_{3}(99.999 \%)$ was purchased from Alfa Aesar. $\mathrm{LiOH} \cdot \mathrm{H}_{2} \mathrm{O}(99.95 \%$, trace metals basis), tetrachloroauric acid trihydrate $\left(\mathrm{HAuCl}_{4} \cdot 3 \mathrm{H}_{2} \mathrm{O}, 99 \%\right)$, tert-butylamine borane (TBAB, 97\%), oleylamine (OLAM_SA, technical grade, 70\%), oleic acid (OA, technical grade, 90\%), 1-octadecene (ODE, technical grade, 90\%), acetic acid (>99\%), toluene, ethanol, and methanol were purchased from Sigma Aldrich. Oleylamine (OLAM_TCI) was purchased from TCI America. OLAM_SA was used as received while OLAM_TCI was pre-dried at $100{ }^{\circ} \mathrm{C}$ under vacuum for four hours and stored inside a $\mathrm{N}_{2}$-filled glovebox. All nanocrystal syntheses were carried out using standard Schlenk techniques under $\mathrm{N}_{2}$ atmosphere.

Synthesis of gadolinium acetate. Typically, $250 \mathrm{~mL}$ of DI water, $50 \mathrm{~mL}$ of ethanol and $40 \mathrm{~g}$ of $\mathrm{Gd}_{2} \mathrm{O}_{3}$ powder were added into a $500 \mathrm{~mL}$ flask and the mixture was heated to $85^{\circ} \mathrm{C}$. Next, $50 \mathrm{~mL}$ of acetic acid was added dropwise to the mixture under stirring until a clear solution was formed. After heating at $85{ }^{\circ} \mathrm{C}$ for additional $30 \mathrm{~min}$, solvents were removed by using a rotary evaporator and the solids were further dried inside a vacuum oven at $50{ }^{\circ} \mathrm{C}$ for $24 \mathrm{~h}$.

Synthesis of $\mathbf{G d}_{2} \mathrm{O}_{3}$ triangular nanoprisms. $\mathrm{Gd}_{2} \mathrm{O}_{3}$ nanoprisms were synthesized according to a previously reported method with modifications. ${ }^{3}$ In a typical reaction, $6 \mathrm{mmol}$ of $\mathrm{LiOH} \cdot \mathrm{H}_{2} \mathrm{O}$, $12 \mathrm{~mL}$ of OA, $18 \mathrm{~mL}$ of OLAM_SA and $30 \mathrm{~mL}$ of ODE were added to a 125-mL three-neck flask at room temperature. The mixture was then heated under vacuum at $110{ }^{\circ} \mathrm{C}$ for $60 \mathrm{~min}$ to form a clear solution. After refilling the reaction flask with $\mathrm{N}_{2}, 3 \mathrm{mmol}$ of gadolinium acetate was added, followed by vacuum degassing at $110^{\circ} \mathrm{C}$ for another $60 \mathrm{~min}$. After refilling with $\mathrm{N}_{2}$, the reaction mixture was heated to $300{ }^{\circ} \mathrm{C}$ for $17.0 \mathrm{~nm}$ prisms or $320^{\circ} \mathrm{C}$ for $23.5 \mathrm{~nm}$ prisms at $20^{\circ} \mathrm{C} / \mathrm{min}$ and kept at that temperature for 60 min under $\mathrm{N}_{2}$ flow. The reaction mixture was air-cooled to approximately $150{ }^{\circ} \mathrm{C}$ when $10 \mathrm{~mL}$ of toluene was added. Nanoprisms were isolated by precipitation with methanol and centrifugation at $3000 \mathrm{rpm}$ for $2 \mathrm{~min}$. After re-dispersing the nanoprisms in $20 \mathrm{~mL}$ of toluene, $10 \mathrm{~mL}$ of methanol was added, and the resulting mixture was 
centrifuged at $3000 \mathrm{rpm}$ for 2 min to remove excess ligands and reaction byproducts such as lithium oleate. Nanoprisms were finally dispersed in $20 \mathrm{~mL}$ of toluene.

Synthesis of Au nanocrystals (NCs). In a typical reaction, $10 \mathrm{~mL}$ of pre-dried OLAM_TCI was loaded into a $100 \mathrm{~mL}$ three-neck flask. After degassing under vacuum for $20 \mathrm{~min}$ at room temperature, $10 \mathrm{~mL}$ of anhydrous toluene was injected into the flask followed by flushing with $\mathrm{N}_{2}$ for $10 \mathrm{~min}$. Afterward, $0.25 \mathrm{mmol}(98 \mathrm{mg})$ of $\mathrm{HAuCl}_{4} \cdot 3 \mathrm{H}_{2} \mathrm{O}$ was added into the mixture followed by purging with $\mathrm{N}_{2}$ for another $20 \mathrm{~min}$. Subsequently, a solution mixture of $0.25 \mathrm{mmol}$ (22 $\mathrm{mg}$ ) of TBAB, 1 mL of OLAM_TCI, and $1 \mathrm{~mL}$ of anhydrous toluene was swiftly injected. The resulting mixture was kept under stirring for another $60 \mathrm{~min}$ at room temperature. Au NCs were isolated via precipitation with $60 \mathrm{~mL}$ of acetone followed by centrifugation at $6000 \mathrm{rpm}$ for $5 \mathrm{~min}$ and redispersed in toluene $(5 \mathrm{mg} / \mathrm{mL})$.

Materials characterization. Low-magnification TEM images were acquired on a JEOL JEM 1400 plus microscope equipped with a $\mathrm{LaB}_{6}$ filament operating at $120 \mathrm{kV}$. Aberration-corrected STEM (AC-STEM) images were recorded on a $200 \mathrm{kV}$ JEOL JEM-ARM200F STEM equipped with spherical aberration correctors. Fourier transform infrared (FTIR) spectra were collected in transmission mode on a Bruker VERTEX 70v FTIR spectrometer at spectral resolution $4 \mathrm{~cm}^{-1}$. FTIR transmittance of different samples was normalized with the mass per unit area of NC inorganic cores of the deposited film assuming a uniform film thickness. Scanning electron microscopy (SEM) images were taken on a Carl Zeiss Auriga 60 FIB-SEM operating at $1.5 \mathrm{kV}$. Powder X-ray diffraction (XRD) patterns were collected on a PANalytical Empyrean X-ray diffractometer operating at $40 \mathrm{kV}$ and $45 \mathrm{~mA}$. XRD samples were prepared by drop-casting NC solutions onto single-crystalline Si substrates. Thermogravimetric analysis (TGA) was performed by using a TA Instruments Q5000 system. Nanocrystals ( $2 \mathrm{mg}$ ) were loaded into a platinum pan that was first heated at $120{ }^{\circ} \mathrm{C}$ for 30 min before raising to $700{ }^{\circ} \mathrm{C}$ at constant heating rate of $10^{\circ} \mathrm{C} /$ min under $\mathrm{N}_{2}$. Data analysis was carried out using the TA Universal Analysis software package. Atomic force microscopy (AFM) images were acquired by using an MFP-3D AFM (Asylum Research) operated in tapping mode. Average molecular weight and polydispersity index of PS-Br were determined using a Waters Breeze GPC system equipped with a Waters 1525 binary HPLC pump, a 2414 refractive index detector, and two connected Styragel high resolution columns (HR4 and HR4E, $7.8 \times 300 \mathrm{~mm}$ ). THF was used as the eluent at flow rate $1 \mathrm{~mL} / \mathrm{min}$ during GPC measurements. The instrument was calibrated using commercial polystyrene standards. ${ }^{1} \mathrm{H}$ NMR spectra were recorded in deuterated chloroform at $298 \mathrm{~K}$ using a Varian $600 \mathrm{MHz}$ Inova spectrometer.

\section{Section 3. Synthesis of polymer-grafted nanocrystals and their assembly into ordered superstructures}


Grafting PS-PEHA onto NCs through a direct ligand-exchange protocol. In a typical process, $\mathrm{Gd}_{2} \mathrm{O}_{3}$ triangular nanoprisms were dispersed in THF at concentration $10 \mathrm{mg} / \mathrm{mL}$. A certain amount of PS-PEHA (Table S3 for details) was dissolved separately into $0.8 \mathrm{~mL}$ of THF. Afterward, 200 $\mu \mathrm{L}$ of the $\mathrm{Gd}_{2} \mathrm{O}_{3}$ nanoprism solution was added into the PS-PEHA solution. Following sonication for $10 \mathrm{~s}$, the solution mixture was left undisturbed for $12 \mathrm{~h}$. PS-grafted $\mathrm{Gd}_{2} \mathrm{O}_{3}$ nanoprisms were purified through precipitation with heptane followed by centrifugation at $2000 \mathrm{rpm}$ for $2 \mathrm{~min}$. The pellet was re-dispersed in THF. This cleaning procedure was repeated one to three times to remove unbound PS-PEHA. $\mathrm{Gd}_{2} \mathrm{O}_{3}$ nanoprisms were finally dispersed in $1 \mathrm{~mL}$ of toluene. The feeding grafting density of PS-PEHA, which is defined as the number of PS-PEHA ligands per unit area based on the mass of nanoprisms and the amount of PS-PEHA used for ligand-exchange, is calculated as

$$
\sigma_{f}=\frac{N_{\mathrm{A}} \times \frac{\sqrt{3}}{4} l^{2} \times h \times \rho \times m_{\mathrm{PS}}}{\left(2 \times \frac{\sqrt{3}}{4} l^{2}+3 \times h \times l\right) \times M W_{\mathrm{PS}} \times m_{\mathrm{NC}}}
$$

where $l$ and $h$ are the average edge length and height of $\mathrm{Gd}_{2} \mathrm{O}_{3}$ triangular nanoprisms, respectively. $\rho$ is the bulk density of $\mathrm{Gd}_{2} \mathrm{O}_{3}\left(7.41 \mathrm{~g} / \mathrm{cm}^{3}\right)^{4-6}$ and $N_{\mathrm{A}}$ is the Avogadro constant. $m_{\mathrm{PS}}$ and $M W_{\mathrm{PS}}$ are the mass and average molecular weight of PS-PEHA used for ligand-exchange, respectively. $m_{\mathrm{NC}}$ is the mass of the inorganic cores of nanoprisms based on TGA.

To graft PS-PEHA onto spherical Au NCs, a certain amount of PS-PEHA (Table S5 for details) was dissolved in $1 \mathrm{~mL}$ of THF, to which $0.2 \mathrm{~mL}$ of Au NC solution (5 mg/mL in toluene) was added. After sonication for 10 seconds, the solution was left undisturbed for $12 \mathrm{~h}$. PS-grafted Au NCs were purified through precipitation with heptane followed by centrifugation at $3000 \mathrm{rpm}$ for $2 \mathrm{~min}$. The pellet was re-dispersed in THF and this cleaning process was repeated one to three times to remove unbound PS-PEHA.

The feeding grafting density of PS-PEHA, which is defined as the number of PS-PEHA ligands per unit area based on the mass of Au NCs and the amount of PS-PEHA used for ligand-exchange is calculated as

$$
\sigma_{f}=\frac{N_{\mathrm{A}} \times r_{\mathrm{NC}} \times \rho \times m_{\mathrm{PS}}}{3 \times M W_{\mathrm{PS}} \times m_{\mathrm{NC}}}
$$

where $r_{\mathrm{NC}}$ is the radius of Au NCs. $\rho=19.3 \mathrm{~g} / \mathrm{cm}^{3}$ is the bulk density of $\mathrm{Au}^{7-9}$ and $N_{\mathrm{A}}$ is the Avogadro constant. $m_{\mathrm{PS}}$ and $M W_{\mathrm{PS}}$ are the mass and average molecular weight of PS-PEHA used for ligand-exchange, respectively. $m_{\mathrm{NC}}$ is the mass of the Au NC cores based on TGA.

Grafting PS-PEHA onto NCs through a two-step ligand-exchange protocol. The two-step ligand-exchange process includes stripping of native alkyl ligands of NCs followed by grafting with PS-PEHA. In the first step, performed inside a $\mathrm{N}_{2}$-purged glovebox using anhydrous solvents, 
$2 \mathrm{~mL}$ of $\mathrm{Gd}_{2} \mathrm{O}_{3}$ nanoprisms dissolved in hexane $(5 \mathrm{mg} / \mathrm{mL})$ was added to a $1 \mathrm{~mL}$ acetonitrile solution of $\mathrm{Et}_{3} \mathrm{OBF}_{4}(0.5 \mathrm{M})$. The biphasic mixture was stirred $12 \mathrm{~h}$ at room temperature, after which nanoprisms were transferred from the upper hexane layer to the bottom acetonitrile layer. After removing the hexane phase using a glass pipette, $\mathrm{Gd}_{2} \mathrm{O}_{3}$ nanoprisms were precipitated by adding toluene to the acetonitrile solution and centrifuged at $3000 \mathrm{rpm}$ for $2 \mathrm{~min}$. Nanoprisms were re-dispersed into DMF forming a stable, transparent solution at the concentration of $10 \mathrm{mg} / \mathrm{mL}$.

In the second step, a certain amount of PS-PEHA (Table S6 for details) was dissolved in a mixture of $1.0 \mathrm{~mL}$ of THF and $0.4 \mathrm{~mL}$ of DMF, to which $0.2 \mathrm{~mL}$ of DMF solution $(10 \mathrm{mg} / \mathrm{mL})$ of ligandstripped $\mathrm{Gd}_{2} \mathrm{O}_{3}$ nanoprisms was added. Following sonication for $10 \mathrm{~s}$, this mixture was left undisturbed for $24 \mathrm{~h}$. PS-grafted $\mathrm{Gd}_{2} \mathrm{O}_{3}$ prisms were recovered through precipitation with heptane followed by centrifugation at $2000 \mathrm{rpm}$ for $2 \mathrm{~min}$. The pellet was re-dispersed in THF. This cleaning procedure was repeated one more time to remove unbound PS-PEHA. $\mathrm{Gd}_{2} \mathrm{O}_{3}$ nanoprisms were finally dispersed in $1 \mathrm{~mL}$ of toluene. The feeding grafting density $\sigma_{f}$ of PS-PEHA can be calculated using equation (1). Because the amount of residual alkyl ligands is negligibly small for the ligand-stripped nanoprisms, the actual grafting density of PS-grafted nanoprisms is calculated based on TGA results as

$$
\sigma=\frac{N_{\mathrm{A}} \times \frac{\sqrt{3}}{4} l^{2} \times h \times \rho \times X}{\left(2 \times \frac{\sqrt{3}}{4} l^{2}+3 \times h \times l\right) \times M W_{\mathrm{PS}} \times(1-X)}
$$

where $l$ and $h$ are the edge length and height of the $\mathrm{Gd}_{2} \mathrm{O}_{3}$ triangular nanoprism, respectively. $\rho$ is the bulk density of $\mathrm{Gd}_{2} \mathrm{O}_{3}\left(7.41 \mathrm{~g} / \mathrm{cm}^{3}\right)^{4-6}$ and $N_{\mathrm{A}}$ is the Avogadro constant. $M W_{\mathrm{PS}}$ is the average molecular weight of PS-PEHA ligands and $X$ is the mass percentage of PS-PEHA ligands determined from TGA.

Self-assembly of PS-grafted $\mathrm{Gd}_{2} \mathrm{O}_{3}$ prisms into ordered superstructures. Ordered NC superstructures were prepared by using two deposition methods.

Deposition method 1. The liquid-air interfacial assembly method involves drying a solution of nanoprisms on top of an immiscible EG liquid subphase. Typically, $20 \mu \mathrm{L}$ toluene solution of PSgrafted $\mathrm{Gd}_{2} \mathrm{O}_{3}$ nanoprisms ( $2 \mathrm{mg} / \mathrm{mL}$ estimated based on the inorganic content) was drop-cast onto the surface of EG inside a Teflon well $\left(1.5 \times 1.5 \times 1.5 \mathrm{~cm}^{3}\right)$. The well was subsequently covered with a glass slide to slow down solvent evaporation. After $5 \mathrm{~h}$, the resulting NC film was transferred onto a carbon-coated $\mathrm{Cu}$ TEM grid, which was further dried inside a vacuum oven to remove residual EG.

Deposition method 2. Ordered superstructures were also prepared by drying PS-grafted $\mathrm{Gd}_{2} \mathrm{O}_{3}$ nanoprisms directly onto $\mathrm{Si}$ substrates. Typically, a Si substrate $\left(0.7 \times 0.7 \mathrm{~cm}^{2}\right)$ was placed on top of a piece of copper ferrule insides a Teflon well $\left(1.5 \times 1.5 \times 1.5 \mathrm{~cm}^{3}\right)$ prefilled with $40 \mu \mathrm{L}$ of 
toluene. Next, $30 \mu \mathrm{L}$ of PS-grafted $\mathrm{Gd}_{2} \mathrm{O}_{3}$ nanoprisms ( $2 \mathrm{mg} / \mathrm{mL}$ in toluene) was drop-cast onto the Si substrate. The Teflon well was covered with a glass slide to slow down solvent evaporation. Samples were usually fully dried after $24 \mathrm{~h}$. To prepare samples suitable for TEM imaging, a carbon-coated Cu TEM grid was placed on top of the Si substrate before drop-casting NC solution.

\section{Section 4. FTIR peak assignments and spectral analysis for as-synthesized and PS- grafted $\mathrm{Gd}_{2} \mathrm{O}_{3}$ nanoprisms}

We used FTIR spectroscopy to qualitatively evaluate the ligand composition for both assynthesized and PS-grafted nanoprisms. For PS-grafted $\mathrm{Gd}_{2} \mathrm{O}_{3}$ nanoprisms made by direct ligandexchange with PS-PEHA, we mainly focus on two spectral regions, namely $3200-2800 \mathrm{~cm}^{-1}$ (region I) and 1600-1400 $\mathrm{cm}^{-1}$ (region II). Relevant data are presented in Fig. $1 \mathrm{~h}$ in the main text.

In region I, peaks in the range $3000-2800 \mathrm{~cm}^{-1}$ arise from saturated aliphatic $\mathrm{C}-\mathrm{H}$ stretching vibrations, ${ }^{10,11}$ and were present in both as-synthesized nanoprisms (e.g., from methylene units of alkyl chains) and PS-grafted nanoprisms (e.g., from the (CH)x moieties of polymer backbone). ${ }^{12}$ We attribute the peak at $3006 \mathrm{~cm}^{-1}$ to $\mathrm{C}-\mathrm{H}$ stretching of the alkene moiety $(=\mathrm{C}-\mathrm{H})$ from OA or OLAM. ${ }^{13-15}$ Peaks in the range of $3200-3020 \mathrm{~cm}^{-1}$ are attributed to the aromatic $\mathrm{C}-\mathrm{H}$ stretching vibrations. ${ }^{12}$

In region II, peak assignments are listed below:

- $1600 \mathrm{~cm}^{-1}: \mathrm{N}-\mathrm{H}$ bending from OLAM or PEHA, ${ }^{11,16}$ or $\mathrm{C}=\mathrm{C}$ stretching from the aromatic ring. ${ }^{12}$

- $1570 \mathrm{~cm}^{-1}$ : asymmetric stretching vibration of bridging bidentate $(\mathrm{O}-\mathrm{C}-\mathrm{O}) \cdot{ }^{17-19}$

- $1496 \mathrm{~cm}^{-1}$ : asymmetric stretching vibration of chelating bidentate $(\mathrm{O}-\mathrm{C}-\mathrm{O})^{18}$ or $\mathrm{C}=\mathrm{C}$ stretching from the aromatic ring. ${ }^{12}$

- $1452 \mathrm{~cm}^{-1}$ : $\mathrm{C}=\mathrm{C}$ stretching vibration from the aromatic ring ${ }^{12}$ or the $\mathrm{CH}_{2}$ in-plane scissoring vibration. ${ }^{11,17,20-23}$

- $\quad 1433 \mathrm{~cm}^{-1}$ : symmetric stretching vibration of $\mathrm{O}-\mathrm{C}-\mathrm{O} \cdot{ }^{18,21,22}$

Discussion:

$>$ The peak at $1600 \mathrm{~cm}^{-1}$ is present in all three spectra of Fig. 1h. For as-synthesized $\mathrm{Gd}_{2} \mathrm{O}_{3}$ nanoprisms, this peak arises from the $\mathrm{N}-\mathrm{H}$ bending absorption of the OLAM ligands. ${ }^{11}$ With PS-PEHA, this peak can be attributed to $\mathrm{C}=\mathrm{C}$ stretching of the aromatic ring or $\mathrm{N}-\mathrm{H}$ bending from the PEHA moiety. ${ }^{12,16}$

The peak at $1452 \mathrm{~cm}^{-1}$ is present in pure PS-PEHA and PS-grafted nanoprisms, but not in as-synthesized nanoprisms. We attribute it to the $\mathrm{C}=\mathrm{C}$ stretching of the aromatic ring of $\mathrm{PS}^{12}$ or the $\mathrm{CH}_{2}$ in-plane scissoring vibration. ${ }^{11,17,20-23}$ 
The peak at $1433 \mathrm{~cm}^{-1}$ is present is as-synthesized and PS-grafted nanoprisms but absent in pure PS-PEHA. We attribute it to symmetric stretch of $\mathrm{O}-\mathrm{C}-\mathrm{O} \cdot{ }^{10,21,22}$

$>$ The peaks at $1570 \mathrm{~cm}^{-1}$ and $1496 \mathrm{~cm}^{-1}$ correspond to the asymmetric stretching vibration of $\mathrm{O}-\mathrm{C}-\mathrm{O} .{ }^{17-19}$ The energy difference between the symmetric and asymmetric $\mathrm{O}-\mathrm{C}-\mathrm{O}$ stretches, $\Delta$, is diagnostic of the carboxylate's binding motif. ${ }^{24-26} \Delta$ is $137 \mathrm{~cm}^{-1}$ between peaks of 1570 and $1433 \mathrm{~cm}^{-1}$, corresponding to a bridging bidentate motif. ${ }^{13,27}$ By contrast, $\Delta$ is $63 \mathrm{~cm}^{-1}$ between peaks of 1496 and $1433 \mathrm{~cm}^{-1}$, corresponding to a chelating bidentate motif. $^{13,27}$

For PS-grafted $\mathrm{Gd}_{2} \mathrm{O}_{3}$ nanoprisms prepared via the two-step ligand-exchange protocol, we mainly focus on three spectral regions, namely $3200-2800 \mathrm{~cm}^{-1}$ (region I), $1750-1400 \mathrm{~cm}^{-1}$ (region II) and $1100-1000 \mathrm{~cm}^{-1}$ (Region III). Relevant data are shown in Figure S30.

In Region I, peaks in the range of $3000-2800 \mathrm{~cm}^{-1}$ result from C-H stretching vibrations, and were present in both as-synthesized alkyl-capped nanoprisms (e.g., methylene units) ${ }^{10,11}$ and PS-grafted nanoprisms (e.g., $(\mathrm{CH})_{\mathrm{x}}$ moieties of the polymer backbone). ${ }^{12}$ The broad feature centered at around $3300 \mathrm{~cm}^{-1}$ likely arises from adsorbed water molecules. ${ }^{28}$ Peaks in the range of $3200-3020 \mathrm{~cm}^{-1}$ are attributed to the aromatic $\mathrm{C}-\mathrm{H}$ stretching vibrations. ${ }^{12}$

In regions II and III, the peak assignments are listed below:

- $1726 \mathrm{~cm}^{-1}$ (\#1) and $1674 \mathrm{~cm}^{-1}$ (\#2): aromatic combination bands. ${ }^{12}$

- $1658 \mathrm{~cm}^{-1}$ (\#3): $\mathrm{C}=\mathrm{O}$ stretching vibration from residual DMF molecules. ${ }^{28}$

- $1600 \mathrm{~cm}^{-1}$ (\#4): $\mathrm{N}-\mathrm{H}$ bending from OLAM or PEHA, ${ }^{11,16}$ or $\mathrm{C}=\mathrm{C}$ stretching from the aromatic ring. $^{12}$

- $1583 \mathrm{~cm}^{-1}$ (\#5), $1492 \mathrm{~cm}^{-1}$ (\#8), and $1452 \mathrm{~cm}^{-1}$ (\#9): C=C stretching vibrations from the aromatic ring. The peak at $1452 \mathrm{~cm}^{-1}$ could also arise from the $\mathrm{CH}_{2}$ in-plane scissoring vibration. ${ }^{11,12,23}$

- $1570 \mathrm{~cm}^{-1}$ (\#6): asymmetric stretching vibration of bridging bidentate $(\mathrm{O}-\mathrm{C}-\mathrm{O}) .{ }^{17-19}$

- $1496 \mathrm{~cm}^{-1}$ (\#7): asymmetric stretching vibration of chelating bidentate $(\mathrm{O}-\mathrm{C}-\mathrm{O}) .{ }^{17-19}$

- $1433 \mathrm{~cm}^{-1}$ (\#10): symmetric stretching vibration of $\mathrm{O}-\mathrm{C}-\mathrm{O} .^{18,21,22}$

- $1084 \mathrm{~cm}^{-1}(\# 11): \mathrm{BF}_{4}^{-}$ions. ${ }^{28,29}$

- $1066 \mathrm{~cm}^{-1}$ (\#12) and $1028 \mathrm{~cm}^{-1}$ (\#13): in-plane C-H bending vibrations of the aromatic ring. ${ }^{12}$

Discussion:

$>$ As shown in Figure S30, peak intensities in region I for $\mathrm{Et}_{3} \mathrm{OBF}_{4}$-treated nanoprisms nearly diminished, indicating that most native alkyl ligands were removed.

$>$ Peaks \#1 and \#2 assigned to aromatic combination bands were present for pure PS-PEHA and PS-grafted nanoprisms. ${ }^{12}$ Peak \#3 was detected only for Et3OBF4-treated nanoprisms.

$>$ Peaks \#4 was present in all four spectra. For as-synthesized $\mathrm{Gd}_{2} \mathrm{O}_{3}$ nanoprisms, it results from the N-H bending absorption of OLAM ligands. ${ }^{11}$ This peak intensity decreased significantly 
for $\mathrm{Et}_{3} \mathrm{OBF}_{4}$-treated nanoprisms. For pure PS-PEHA and PS-grafted nanoprisms, this peak can be attributed to $\mathrm{C}=\mathrm{C}$ stretching of the aromatic ring and the $\mathrm{N}-\mathrm{H}$ bending from the PEHA moiety. ${ }^{12,16}$

$>$ Peaks \#6 and \#7 were still present for Et3 $\mathrm{OBF}_{4}$-treated nanoprisms, even though the weak peak intensities of region I indicate nearly complete removal of native alkyl ligands. We attribute these peaks in $\mathrm{Et}_{3} \mathrm{OBF}_{4}$-treated nanoprisms to carbonate species $\left(\mathrm{CO}_{2}^{3-}\right)$ that resulted from adsorption of ambient $\mathrm{CO}_{2} .^{10}$

$>$ Peak \#11 was detected only for $\mathrm{Et}_{3} \mathrm{OBF}_{4}$-treated nanoprisms.

\section{Section 5. Calculations of pairwise van der Waals (vdW) interaction between $\mathrm{Gd}_{2} \mathrm{O}_{3}$ triangular nanoprisms using a coarse-grained model}

To calculate the pairwise vdW interaction between the inorganic cores of $\mathrm{Gd}_{2} \mathrm{O}_{3}$ nanoprisms, we develop a coarse-grained model ${ }^{30,31}$ where individual prisms are discretized into small cubic meshes with edge length $d=0.4 \mathrm{~nm}$. The pairwise vdW interaction between two meshes is ${ }^{30,31}$

$$
U_{\mathrm{vdW}}(r)=-A \frac{d^{6}}{\pi^{2} r^{6}}
$$

where $r$ is the center-to-center distance between two meshes and $A$ is the Hamaker constant of $\mathrm{Gd}_{2} \mathrm{O}_{3}$ interacting in a hydrocarbon medium. The parameter $A$ can be estimated as ${ }^{32}$

$$
A=A_{131} \approx\left(\sqrt{A_{11}}-\sqrt{A_{22}}+\sqrt{A_{22}}-\sqrt{A_{33}}\right)^{2}=\left(\sqrt{A_{121}}+\sqrt{A_{22}}-\sqrt{A_{33}}\right)^{2}=3.5 \times 10^{-20} \mathrm{~J}
$$

where $A_{121}=6.8 \times 10^{-20} \mathrm{~J}$ is the Hamaker constant of $\mathrm{Gd}_{2} \mathrm{O}_{3}$ in water, ${ }^{33}$ and $A_{22}=3.4 \times 10^{-20} \mathrm{~J}$ and $A_{33}=7.1 \times 10^{-20} \mathrm{~J}$ are the Hamaker constants of water and hydrocarbon in vacuum. ${ }^{32} \mathrm{vdW}$ interactions between discretized mesh elements are assumed to be additive, and thus the total pairwise vdW interaction between two $\mathrm{Gd}_{2} \mathrm{O}_{3}$ nanoprisms of different configurations is

$$
E(r)=\sum_{i=1}^{N} \sum_{j=1}^{N}-A \frac{d^{6}}{\pi^{2}\left|\boldsymbol{r}_{i}-\boldsymbol{r}_{j}\right|^{6}}
$$

where $N$ is the number of meshes for one prism and $\left|\boldsymbol{r}_{i}-\boldsymbol{r}_{j}\right|$ is the distance between the meshes $i$ and $j$. The sums over $i$ and $j$ run over all meshes of the two triangular nanoprisms. We verified that using a mesh size of $0.4 \mathrm{~nm}$ is sufficient to ensure convergence of the summation while being computationally efficient. For instance, the difference between calculated vdW interaction values for a pair of face-to-face aligned nanoprisms is less than $3 \%$ when using a mesh size of $0.4 \mathrm{~nm}$ versus $0.2 \mathrm{~nm}$. As shown in Figure S5, the vdW interactions of edge-to-edge, edge-to-vertex and vertex-to-vertex configurations are negligibly small compared with the face-to-face configuration. 


\section{Section 6. Calculations of ligand-ligand interactions between $\mathrm{Gd}_{2} \mathrm{O}_{3}$ triangular nanoprisms}

We also examine the ligand interaction energies for as-made (OA-capped) and PS-grafted $\mathrm{Gd}_{2} \mathrm{O}_{3}$ nanoprisms in the dry state. The vdW dispersion interactions between two parallel interdigitated ligands can be calculated as ${ }^{34-36}$

$$
E_{\mathrm{vdW}}(d)=-C \times \frac{3 \pi}{8 \lambda^{2}} \times \frac{(2 L-d)}{D^{5}}
$$

where $C$ is the coefficient of the dispersion interaction between two basic units (the London constant), $\lambda$ is the contour length of the repeating unit, $L$ is the ligand length in the solvated state, $d$ is the surface-to-surface distance between adjacent nanoprisms of close-packed arrays, and $D$ is the inter-chain spacing between two interdigitated ligands. In this model, the vdW dispersion interaction between two non-overlapping ligand chains is negligible.

The elastic repulsion energy between two compressed ligands is given by ${ }^{35}$

$$
E_{\text {ela }}=\frac{1}{2} \times \frac{E A_{0}}{L} \times(2 L-d)^{2}
$$

where $E$ is the elastic modulus of ligand molecules. $A_{0}$ is the cross-sectional area upon which the compressive force is applied.

(1) For oleic acid, $C=1.33 \times 10^{-77} \mathrm{~J} \cdot \mathrm{m}^{6}, \lambda=0.127 \mathrm{~nm}, L=2 \mathrm{~nm}, D=0.49 \mathrm{~nm}, E=1 \mathrm{GPa}$, $A_{0}=0.25 \mathrm{~nm}^{2} .{ }^{34,35,37}$ The calculated ligand interaction energies as a function of NC surface-tosurface distance are shown in Figure S6b. An energy minimum of $-0.83 k_{\mathrm{B}} T$ is found at $3.8 \mathrm{~nm}$. This result is in good agreement with our experimental results (Figure S9).

(2) For PS, $\lambda=0.25 \mathrm{~nm}, D=0.4 \mathrm{~nm}, E=3 \mathrm{GPa}, A_{0}=0.64 \mathrm{~nm}^{2}{ }^{36,37} C$ is calculated based on the Hamaker constant of PS, the Hamaker constant of PS $\left(A=6.50 \times 10^{-20} \mathrm{~J}\right)$, using the relationship $A=\pi^{2} \times \rho^{2} \times C \cdot{ }^{32}$ Here, $\rho$ is the number density of the repeating unit, which is calculated as $\rho=\rho_{0} / M_{0} \times N_{\mathrm{A}}=6.07 \times 10^{27} \mathrm{~m}^{-3} \cdot M_{0}=104.15 \mathrm{~g} / \mathrm{mol}$ is the molar mass of PS repeating units, $\rho_{0}=1.05 \mathrm{~g} / \mathrm{cm}^{3}$ is the density of PS and $N_{\mathrm{A}}$ is Avogadro's number. The length $L$ of PS ligands is estimated based on dynamic light scattering (DLS) measurement results of spherical $4.5 \mathrm{~nm}$ Au NCs densely grafted with the same PS ligands: $L=2.5 \mathrm{~nm}$ for $1.8 \mathrm{kDa}$ PS, $L$ $=2.9 \mathrm{~nm}$ for $2.6 \mathrm{kDa}$ PS and $L=3.4 \mathrm{~nm}$ for $3.4 \mathrm{kDa}$ PS. The calculated interaction energies for different PS ligands as a function of NC surface-to-surface distance are shown in Figure S6c-e. The energy minima are found at $4.6 \mathrm{~nm}, 5.3 \mathrm{~nm}$, and $6.2 \mathrm{~nm}$ for $1.8 \mathrm{kDa}, 2.6 \mathrm{kDa}$, and $3.4 \mathrm{kDa}$ PS ligands, respectively. In the solid state, vdW attractions between the $\mathrm{Gd}_{2} \mathrm{O}_{3}$ cores are basically negligible compared with interactions from the ligands. 


\section{Section 7. Density functional theory (DFT) calculations of ligand binding on $\mathrm{Gd}_{2} \mathrm{O}_{3}$ triangular nanoprisms}

We performed DFT calculations to assess the binding energies of oleic acid (or oleylamine) on the (001) (or (100)) surface of $\mathrm{Gd}_{2} \mathrm{O}_{3}$ triangular nanoprisms. Our DFT calculations were based on the projector augmented wave (PAW) method ${ }^{38}$ implemented using the Vienna $a b$ initio simulation package (VASP). ${ }^{39}$ The Perdew-Burke-Ernzerhof (PBE) approximation was used to describe the exchange and correlation functionals. ${ }^{40}$ The plane-wave cutoff energy was set to $400 \mathrm{eV}$, the Hellmann-Feynman force on each atom was smaller than $0.01 \mathrm{eV} \AA^{-1}$, and the total energy converged to $10^{-5} \mathrm{eV}$ for structural optimization. $\mathrm{Gd}_{2} \mathrm{O}_{3}$ was modelled by a $2 \times 2$ supercell and a vacuum space of $20 \AA$ was added between adjacent supercells. $\mathrm{Gd}_{2} \mathrm{O}_{3}(001)$ and (100) surfaces were modelled by a five-atomic-layer slab and a six-atomic-layer slab, respectively. Atoms of the upper two layers were allowed to relax while those of the lower layers were fixed at their bulk positions. The $12 \times 12 \times 1$ gamma-centered Monkhorst-Pack $k$-point mesh was used.

To increase computational efficiency, methylamine, formate, or formic acid was substituted for oleylamine, oleate, or oleic acid, because ligands coordinate to NCs only through the end functional group. ${ }^{41-43}$ The binding energy of an adsorbate $E_{\text {ads }}$ is given by

$$
E_{\text {ads }}=E_{\text {adsorbate }+ \text { slab }}-E_{\text {slab }}-E_{\text {adsorbate }}
$$

where $E_{\text {adsorbate+slab }}, E_{\text {slab }}$, and $E_{\text {adsorbate }}$ represent the relaxed total energy of the whole system (i.e., adsorbate plus $\mathrm{Gd}_{2} \mathrm{O}_{3}$ slab), the energy of the (001) (or (100)) surface of $\mathrm{Gd}_{2} \mathrm{O}_{3}$, and the energy of the adsorbate, respectively. As a result, more strongly bound adsorbates correspond to more negative binding energies. Both chelating and bridging bidentate motifs were considered for formate adsorbate.

Results of DFT calculations are presented in Table S4. It is found that the binding energy of different adsorbates on the $\mathrm{Gd}_{2} \mathrm{O}_{3}$ (001) surface is generally higher than that on the (100) surface, indicating that ligands bind more strongly to the basal planes of the triangular prism than its side planes. Specifically, methylamine has lower binding energies compared with formate or formic acid, suggesting that for as-synthesized $\mathrm{Gd}_{2} \mathrm{O}_{3}$ nanoprisms, OLAM binds weaker to $\mathrm{Gd}_{2} \mathrm{O}_{3}$ compared to $\mathrm{OA}$ as ligands. Furthermore, formate on $\mathrm{Gd}_{2} \mathrm{O}_{3}(001)$ surface has the highest binding energy among different combinations of adsorbate and $\mathrm{Gd}_{2} \mathrm{O}_{3}$ surfaces examined, being $-1.647 \mathrm{eV}$ for the bridging bidentate mode and $-1.338 \mathrm{eV}$ for the chelating bidentate mode. By contrast, the chelating bidentate mode is unstable for formate adsorbed on the $\mathrm{Gd}_{2} \mathrm{O}_{3}(100)$ surface. Upon relaxation, formate on the $\mathrm{Gd}_{2} \mathrm{O}_{3}$ (100) surface adopts a bridging bidentate binding mode with the binding energy of $-1.173 \mathrm{eV}$. Taken together, DFT calculations suggest that (1) compared with oleate, the oleylamine ligands of $\mathrm{Gd}_{2} \mathrm{O}_{3}$ nanoprisms can be more easily displaced 
during ligand-exchange with PS-PEHA, and (2) oleate binds more strongly to the (001) basal planes than the (100) side planes of nanoprisms.

\section{Section 8. TEM image processing and analysis to quantify translational and orientational order of nanoprism superstructures}

A simplified flowchart of the image processing and analysis procedure is presented in Figure S18. Raw TEM images were processed by using the open-source software ImageJ (Figure S19). ${ }^{44}$ First, a low-pass filter was applied to reduce image noise by using the Gaussian Blur tool. Next, a threshold value was carefully selected to ensure that the contours of individual NCs were identified accurately. The image was then binarized and the Analyze Particles tool was used to determine the size and the coordinates of the centroid of each NC. Lastly, the retrieved centroid locations were overlaid with the original TEM image using a custom written MATLAB script to check the accuracy of particle centroid detection.

The spatial distribution of NCs for each superstructure was quantified by calculating the radial distribution function $g(r)$ using customized MATLAB codes. $g(r)$ gives the probability of finding another particle at a given distance $r$ from the reference particle and is defined as ${ }^{45}$

$$
g(r)=\frac{\rho(r)}{\rho_{0}}
$$

where $\rho(r)$ is the particle number density at the distance $r$ and $\rho_{0}$ is the average particle number density (Figure S20). A periodic boundary correction was applied to account for the limited field-of-view of TEM images. ${ }^{46}$ Peak linewidths and the spatial decay of $g(r)$ were correlated with the degree of translational order. We set the first minimum after the first peak of $g(r)$ as the threshold of the first nearest-neighbor distance. ${ }^{30,47}$ Likewise, the second and the third nearest-neighbor bond lengths were defined by locating the second and third peaks of $g(r)$. For cases where peaks of $g(r)$ were strongly overlapping and bond length alone became inadequate to differentiate different neighboring NCs, dual criteria of bond length and bond angle was used. The bond networks of the p6m-type superstructure based on different types of nearest neighbors (i.e., first, second and third nearest neighbors) are shown in Figure S20.

To quantify the local translational order of nanoprism superstructures, bond-orientational order parameters were used. The six-fold bond-orientational order parameter $\psi_{6 j}$ for a twodimensional system is given by ${ }^{30,48-50}$

$$
\psi_{6 j}=\frac{1}{N} \sum_{k=1}^{N} \exp \left(i 6 \theta_{j k}\right)
$$


where $i$ is the imaginary unit, $\theta_{j k}$ is the angle between the bond connecting particle $j$ and its nearest neighbor particle $k$ and an arbitrary reference axis, and $N$ is the number of nearest neighbors of particle $j$. Likewise, $\psi_{4 j}$ was used to characterize the four-fold symmetry of the superstructures. $\left|\psi_{6 j}\right|=1\left(\left|\psi_{4 j}\right|=1\right)$ indicates perfect hexagonal (tetragonal) order, whereas $\left|\psi_{6 j}\right|=0 \quad\left(\left|\psi_{4 j}\right|=0\right)$ corresponds to the lack of 6-fold (4-fold) bond-orientational order.. Representative TEM images of the p6m-type superstructure color-coded according to the value of $\left|\psi_{6 j}\right|$ (and $\left|\psi_{4 j}\right|$ ) indicate a high (low) six-fold (four-fold) bond-orientational order (Figure S20hi). Furthermore, the global bond-orientational order parameters can be calculated as ${ }^{51-53}$

$$
\Psi_{4}=\left|\left\langle\psi_{4 j}\right\rangle\right| \quad \text { and } \quad \Psi_{6}=\left|\left\langle\psi_{6 j}\right\rangle\right|
$$

where the angled brackets represent averaging over all particles on the TEM image. The pmg-type structure is characterized by intermediate values of six-fold (four-fold) bond-orientational order parameter $\psi_{6 j}\left(\psi_{4 j}\right)$, which are higher than that of the $\mathrm{p} 2$ phase (Fig. S21). The p6m phase has an essentially unity $\psi_{6 j}$ and a close-to-zero $\psi_{4 j}$, corresponding to nearly perfect hexagonal order. Considering the three-fold symmetry of triangles, not all neighbors can be aligned antiparallel when the coordination number exceeds three (e.g., the pmg-type structure in Fig. 2k).

To quantify the orientational order of nanoprism superstructures, we define the angle $\theta$ as the angle between the horizontal $x$-axis and the line connecting the center and one vertex of individual triangular nanoprisms (Figure S20m). Only angles that fall into the range of $0^{\circ}$ and $120^{\circ}$ were selected owing to the three-fold rotational symmetry of triangles. Moreover, the three-fold orientational correlation function $G_{3}(m)$ was calculated as ${ }^{30,53}$

$$
G_{3}(m)=\left\langle\cos \left(3\left(\theta_{i}-\theta_{i+m}\right)\right\rangle\right.
$$

where $\theta_{i}$ is the angle of the reference particle and $\theta_{i+m}$ is the angle of an $m$-th nearest neighbor. The angled brackets denote averaging over all pairs of reference particles and its $m$-th nearest neighbors. $G_{3}(1)=1(-1)$ indicates that all nearest neighbor triangles are parallel (anti-parallel) aligned. Representative TEM image of the p6m-type superstructures with individual nanoprisms color-coded according to their $G_{3}(\mathrm{~m})$ values clearly show the antiparallel alignment between nearest neighbors (Figure S20n). Furthermore, the global $m$-fold orientational order parameter can be calculated as ${ }^{53,54}$

$$
\Phi_{m}=\left|\left\langle\exp \left(i m \theta_{j}\right)\right\rangle\right|
$$

where $\theta_{j}$ is the angle of particle $j$ with $0^{\circ} \leq \theta_{j} \leq 120^{\circ}$. We used the combination of $\Phi_{3}$ and $\Phi_{6}$ to characterize the orientational order of superstructures. $\Phi_{3}=\Phi_{6}=0$ indicates a fully orientationally amorphous or an ideal plastic phase, whereas $\Phi_{3}=0$ and $\Phi_{6}=1$ is expected for perfectly orientationally ordered superstructures with wallpaper groups $\mathrm{p} 6 \mathrm{~m}$, pmg, and p2. 


\section{Section 9. Calculation of densest packings}

Spherotriangles are defined mathematically as the Minkowski sum of a triangle and a disk. They have two geometric parameters, triangle edge length $\ell$ and disk radius $r_{D}$. The dimensionless rounding parameter is then defined as $R=r_{D} /\left(\ell+r_{D}\right)$ with $R=0$ corresponding to a triangle and $R=1$ corresponding to a disk. Additionally, we will commonly use the height $h=\ell \sqrt{3} / 2$, the area of the triangle $A_{\mathrm{T}}(\ell)=\ell^{2} \sqrt{3} / 4$, and the area of the spherotriangle $A_{\mathrm{ST}}\left(\ell, r_{D}\right)=$ $A_{T}(\ell)+3 \ell r_{D}+\pi r_{D}^{2}$. Details of the geometric construction in this Section are shown in Figure S23. We consider each symmetry separately.

p6m. The particle centers form a regular hexagon with side length $a_{\mathrm{Hex}}\left(\ell, r_{D}\right)=2 r_{D}+2 / 3 h$. Its area is $A_{\mathrm{Hex}}\left(\ell, r_{D}\right)=6 A_{\mathrm{T}}\left(a_{\mathrm{Hex}}\right)$. Each spherotriangle contributes one third of its area to the hexagon, thus the highest packing of $\mathrm{p} 6 \mathrm{~m}$ is

$$
\phi_{\mathrm{p} 6 \mathrm{~m}}\left(\ell, r_{\mathrm{D}}\right)=\frac{2 A_{\mathrm{ST}}\left(\ell, r_{\mathrm{D}}\right)}{A_{\mathrm{Hex}}\left(\ell, r_{\mathrm{D}}\right)} .
$$

pmg. A shaded rectangle in Figure S23 characterizes the unit cell area of the pmg phase. In a flat configuration, the particles centers are displaced vertically by $h / 3$. The height is the same as that of the spherotriangle, $h_{\mathrm{pmg}}=h+2 r_{D}$. The width is the sum of the half-triangle width $\ell / 2$ and the spacing added due to the disk, $2 r_{D} / \cos (\pi / 6)$. Due to the tilt of the spherotriangle sides, we have the additional factor $1 / \cos (\pi / 6)$. Thus $w_{\mathrm{pmg}}=\ell / 2+2 r_{D} / \cos (\pi / 6)$. So far, the highest packing is

$$
\phi_{\text {flat pmg }}\left(\ell, r_{\mathrm{D}}\right)=\frac{A_{\mathrm{ST}}\left(\ell, r_{\mathrm{D}}\right)}{w_{\mathrm{pmg}} h_{\mathrm{pmg}}} .
$$

In the non-flipped configuration, this equation remains valid as long as the spherotriangle sides within a row are in touch, or $\ell \geq r_{D}$.

In the flipped configuration, the equation remains valid as long as the spherotriangle sides between rows remain in touch, or $\ell / 2 \geq 4 R / \sqrt{3}$. With $x=4 R / \sqrt{3}-\ell / 2$ the sideways spacing between spherotriangles of different rows requires a correction. Let $k=x / \tan (\pi / 3)$ and $l=k-2 r_{D}$. With the law of cosines, we get $z=\sqrt{3} h / 2+\sqrt{3 l^{2}-4\left(l^{2}-4 R^{2}\right)} / 2$. If we move every other column of spherotriangles upwards by $s_{v}=k-z \sqrt{3} / 2$, the width can be reduced to $w_{\text {pmg }}^{\prime}=$ $\ell / 2+z / \tan (\pi / 6)$. The resulting packing fraction is

$$
\phi_{\text {rounded pmg }}\left(\ell, r_{\mathrm{D}}\right)=\frac{A_{\mathrm{ST}}\left(\ell, r_{\mathrm{D}}\right)}{w_{\mathrm{pmg}}^{\prime} h_{\mathrm{pmg}}} \text {. }
$$


p2. With a bit of sliding, non-flipped pmg can be compressed further. As a first step, we slide the rows along each other. We gain some space to move columns in the $30^{\circ}$ direction and compress the structure. In the compressed structure, spherotriangles that start in vertex-to-vertex contact will also be in contact with a triangle neighboring their first contact. From the geometry (Figure S23), we learn that the vertical shift is $s_{v}=(2-2 \sqrt{2 / 3}) R$, the length of the second shift is $l_{2}=$ $s_{v} / \sin (\pi / 3)$, and the length of the horizontal shift is $l_{1}=(4-2 \sqrt{2 / 3}) / \sqrt{3} R$. In the first step, we shear lines without changing the area of the unit cell. In the second step, we translate the plane $\mathbf{p}=\left(w_{\text {flat pmg }}+l_{1}, h_{\text {flat pmg }}\right)$ by $\mathbf{d}=l_{2}(\sin (\pi / 3), \cos (\pi / 3))$. The area is reduced by the enclosed parallelogram, $\Delta A=|\mathbf{p} \times \mathbf{d}|$. The packing fraction is

$$
\phi_{\mathrm{p} 2}\left(\ell, r_{\mathrm{D}}\right)=\frac{A_{\mathrm{ST}}\left(\ell, r_{\mathrm{D}}\right)}{w_{\mathrm{pmg}} h_{\mathrm{pmg}}-\Delta A}
$$

\section{Section 10. Determination of the hard spherotriangle phase diagram}

Local order characterization. To identify wallpaper group symmetry and structure in simulations, we look at local order. With the shortest observed particle center-to-particle center distance $r_{\text {min }}$, we consider all bonds that are shorter than $1.3 r_{\min }$. These bonds form a polygonal network. Polygons are easily distinguished if their numbers of vertices are different (Figure S24). Hexagons dominate in both the p6m phase and the p2 phase. To distinguish these hexagons, we analyze rotational symmetry. For every rotation $\varphi_{i}=i \pi / 3$ with $i=0,1, \ldots, 5$, we calculate the mean squared distance $\left\langle d^{2}\right\rangle$ from the point it should coincide with. If $\left\langle d^{2}\right\rangle<r_{\text {min }}^{2} / 4$ (respectively $\left\langle d^{2}\right\rangle<0.06\left(\ell+r_{\min }\right)^{2}$ for hard particle systems), a rotation is considered to match sufficiently well.

In the pmg phase, particle centers form triangles and parallelograms, in the plastic phase mainly triangles. If the orientations match well enough, the polygon is assigned the pmg phase, otherwise it is assigned the plastic phase. Ordered structures of triangles contain two orientations. In combination with the three-fold symmetry of triangles, orientation angles are considered up to a modulo of $60^{\circ}$. To decide whether orientations match well enough, we pick one orientation as reference. Orientations are rotated according to six-fold symmetry such that they are closest to the reference. From these, the mean and variance $\left\langle\varphi^{2}\right\rangle$ are calculated. If $\left\langle\varphi^{2}\right\rangle /(\pi / 3)^{2}<0.25^{2} / 6$ (respectively $\left\langle\varphi^{2}\right\rangle /(\pi / 3)^{2}<0.2^{2} / 6$ for hard particle systems), the fluctuations of orientations are low enough to exclude the plastic phase. Note that $\left\langle\varphi^{2}\right\rangle$ is well-defined only if the orientations fluctuate weakly, which is the only case we are interested in here.

Once all possible triangles, parallelograms, and hexagons have been analyzed in each configuration of particles under investigation, we count all polygon types. If more than 25\% matches are found compared to the number expected in a reference configuration (among the 
phases p6m, pmg, p2, plastic), the configuration is considered as detected. If no reference reaches that threshold, the system is considered as disordered.

Monte Carlo simulations with the hard spherotriangle model. We perform hard particle Monte Carlo simulations using the HPMC ${ }^{55}$ package in HOOMD-blue. ${ }^{56}$ Particles have the shape of spherotriangles. All simulations are initialized in the pmg phase and performed in the isochoric ensemble with flexible shear and aspect ratio changes enabled using the boxMC updater to minimize finite-size effects. Phase diagrams are generated by analyzing the local symmetry of all particles at the end of each simulation. Examples of the analysis are shown in Figure S25.

\section{Section 11. Coarse-grained simulations of attractive triangles}

The literature discusses several models for interacting polymer ligand brushes with two main trends: exclusively repulsive ${ }^{57,58}$ and attractive. ${ }^{58-61}$ Attraction can be the result of van der Waals forces and has also been attributed to entropic effects, possibly mediated by the solvent, for example in simulations of nanoparticles with oleic acid ligands ${ }^{59,60}$ and between nanowires. ${ }^{60}$ Attraction is particularly common for short ligand chains and at high ligand grafting density with the length scale of attraction comparable to the contour length of the ligand molecule, although ligands need not remain straight at low grafting density. In any case, the strength of interactions between nanoparticles, both attractive and repulsive, grows to good approximation linearly with the contact area between the ligand shells.

We model the interaction between two triangular nanoprisms using the Derjaguin approximation ${ }^{62}$, which evaluates the interaction of two bodies using an isotropic pair potential $U_{\text {pair }}$. The Derjaguin approximation approximates the interacting areas by a set of parallel plates, for which the interaction is known. The approximation assumes that the effective interaction energy $U$ is the integral over the interacting area,

$$
U=\int d x \int d y U_{\text {pair }}(h(x, y)),
$$

with $h(x, y)$ as a function of spacing at point $(x, y)$.

For all given literature examples, symmetries define the plane of the integral. For arbitrarily rotated triangles no such symmetries exist. To avoid an influence from an arbitrary choice of plane, we modify the integral. Inspired by van der Waals interaction, the integral is computed over the surface of both shapes A and B,

$$
U=\oint_{\mathrm{A}} d \mathbf{r}_{\mathrm{A}} \oint_{\mathrm{B}} d \mathbf{r}_{\mathrm{B}} U_{\text {pair }}\left(\left|\mathbf{r}_{\mathrm{B}}-\mathbf{r}_{\mathrm{A}}\right|\right)
$$


We can also define an energy per surface area of the ligand brush on one of the shapes (here chosen is shape $A)$ as $u_{A}=\oint_{\mathrm{B}} d \mathbf{r}_{\mathrm{B}} U_{\text {pair }}\left(\left|\mathbf{r}_{\mathrm{B}}-\mathbf{r}_{\mathrm{A}}\right|\right)$. This energy per surface area is shown in Fig. 3d.

The exact form of the pair potential $U_{\text {pair }}$ is not essential for our results. But we know that short ligands and brushes with high grafting densities are typically attractive in the absence of NC charging, ${ }^{59,60}$ and that the softness of a polymer brush varies with length of the chains. ${ }^{58}$ We can thus assume repulsion at short distances, moderate attraction at intermediate distances, and need a parameter to control the width of the attractive well. All these conditions are fulfilled by the Morse potential, which is our potential of choice in this work. The Morse potential has been used in previous works successfully ${ }^{63}$. It is given by

$$
U_{\text {pair }}(r)=U_{0}\left(e^{-2 \alpha\left(r-r_{m}\right)}-2 e^{-\alpha\left(r-r_{m}\right)}\right) .
$$

The Morse potential has three parameters:

(1) The minimum distance $r_{m}$ should be close to $2 r_{D}$ when considering the interaction of two nearly hard spherotriangles. Instead of $r_{m}$, we use the dimensionless brush parameter rounding $R=r_{m} /\left(2 \ell+r_{m}\right)$ in the main text. Rounding $R$ was introduced already in Section 9 for hard spherotriangles. It is a function of ligand brush thickness, which is affected in experiment by the chain parameters ligand length and grafting density. For high grafting density, brush thickness is expected to be comparable to ligand length. But for mid to low grafting density, ligands may bend, and the effective brush thickness can become smaller than ligand length. Chain dispersity and chain distribution may also affect the rounding parameter.

(2) The parameter $\alpha$ is an inverse potential well width. Instead of $\alpha$, we use the dimensionless brush parameter $S=(\alpha \ell)^{-1}$ in the main text. We term $S$ the softness of the ligand brush in the attractive triangle model. In the interaction potential, softness describes the sharpness of the transition from steric repulsion to vdW attraction. Mapped to experiment, $S$ is a function of all four chain parameters ligand length, grafting density, chain dispersity, and chain distribution.

(3) The attraction strength $U_{0}$ introduces an energy scale. It is only relevant when considering thermal motion. Attraction strength can change as a function of ligand chain parameters, in particular ligand length and grafting density. We expect attraction strength to change, possibly by orders of magnitude, during the drying process as the solvent leaves the system. From the observation that NCs are well dispersed during the early stage of drying but eventually form ordered films and free-standing supracrystals during the drying process, we know that attraction strength must increase significantly during our drying experiments. 
We solve the integral in Eq. (5) numerically in preparation of the molecular dynamics simulation with Monte Carlo integration. For this, we create a precomputed table to store the interaction potential. The force is calculated using linear interpolation between tabulated energy values. The tables contain 400 entries for the center-to-center distance and 180 entries for the orientation of each triangle, where we do consider the three-fold symmetry of the particles. A visualization of the tabulated interaction in form of a pair potential map is shown in Figure S26 with a particular choice of relative orientation included in the main text as Fig. 3c.

An equivalent interaction potential may be constructed with a multi-sphere approach in DEM. Instead of solving the integral numerically, the outline of the triangle would then be approximated by discrete interaction points. Every pair of points on the two interacting triangles interacts with the potential $U_{\text {pair }}(r)$. The DEM approach is superior for small systems, or to consider many different parameters. For large systems and when considering many thermodynamic conditions (i.e., temperature, pressure) the overhead of precomputing all possible interactions as conducted by our tabulation approach is negligible compared to the time needed to evaluate interactions during the simulation.

Simulations of the attractive triangle model were performed with an in-house molecular dynamics code. Simulations were initialized in the pmg phase and ran at fixed simulation box volume. The aspect ratio of the simulation box was kept variable by including Monte Carlo box update moves. The system was slowly thermalized to the desired temperature with a Bussi-Donadio-Parrinello thermostat ${ }^{64}$ to allow sufficient time for melting and recrystallization with a different wallpaper group. 

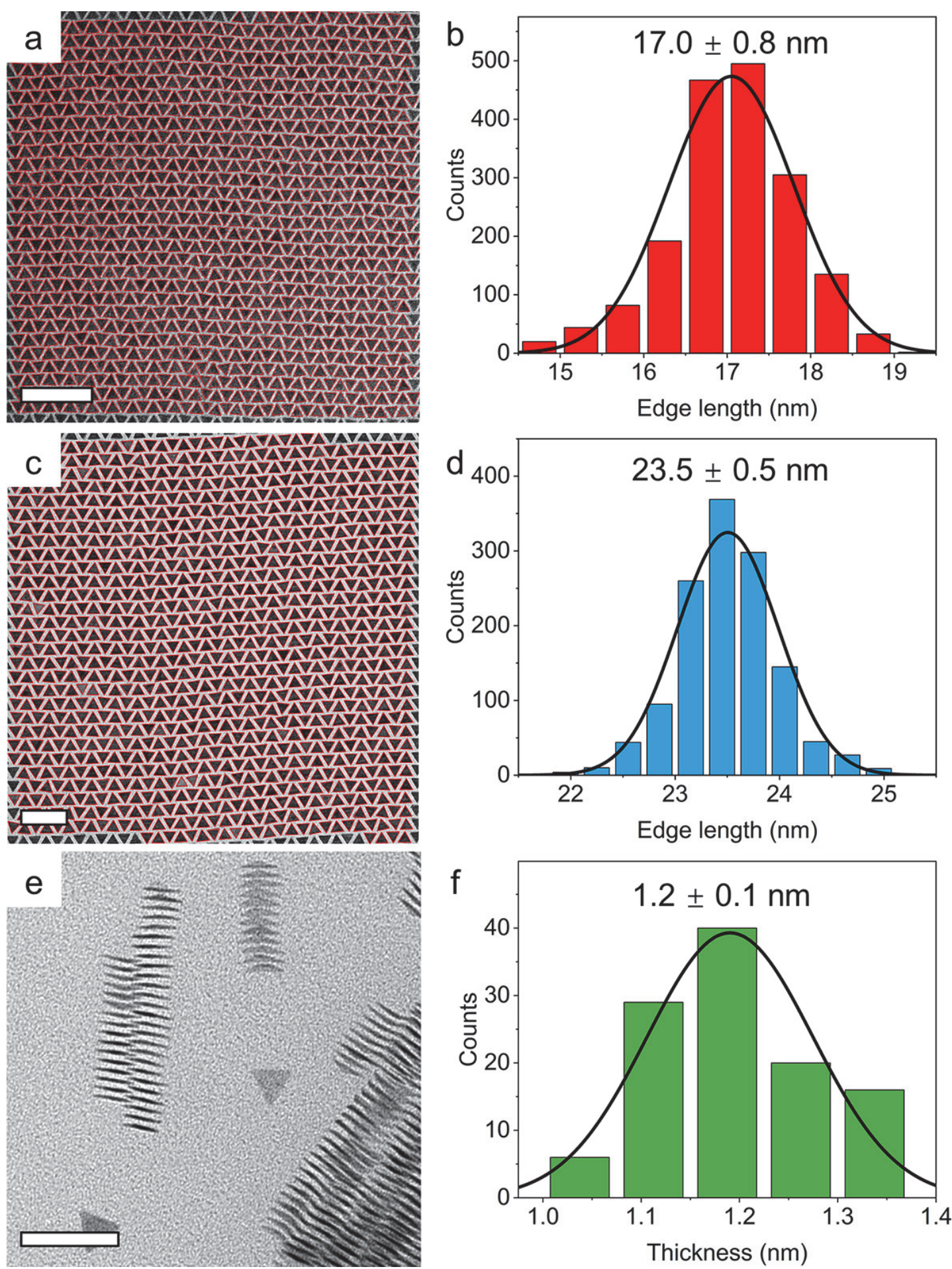

Figure S1. Statistical analysis of nanoprism size based on particle tracking of TEM images. Representative TEM image and size distribution histograms for (a,b) small and (c,d) large triangular nanoprisms. (e,f) Representative TEM image and thickness distribution histogram of large nanoprisms. The black curves represent a Gaussian fit to the corresponding distribution histogram. Scale bars: (a,c) $100 \mathrm{~nm}$; (e) $50 \mathrm{~nm}$. 

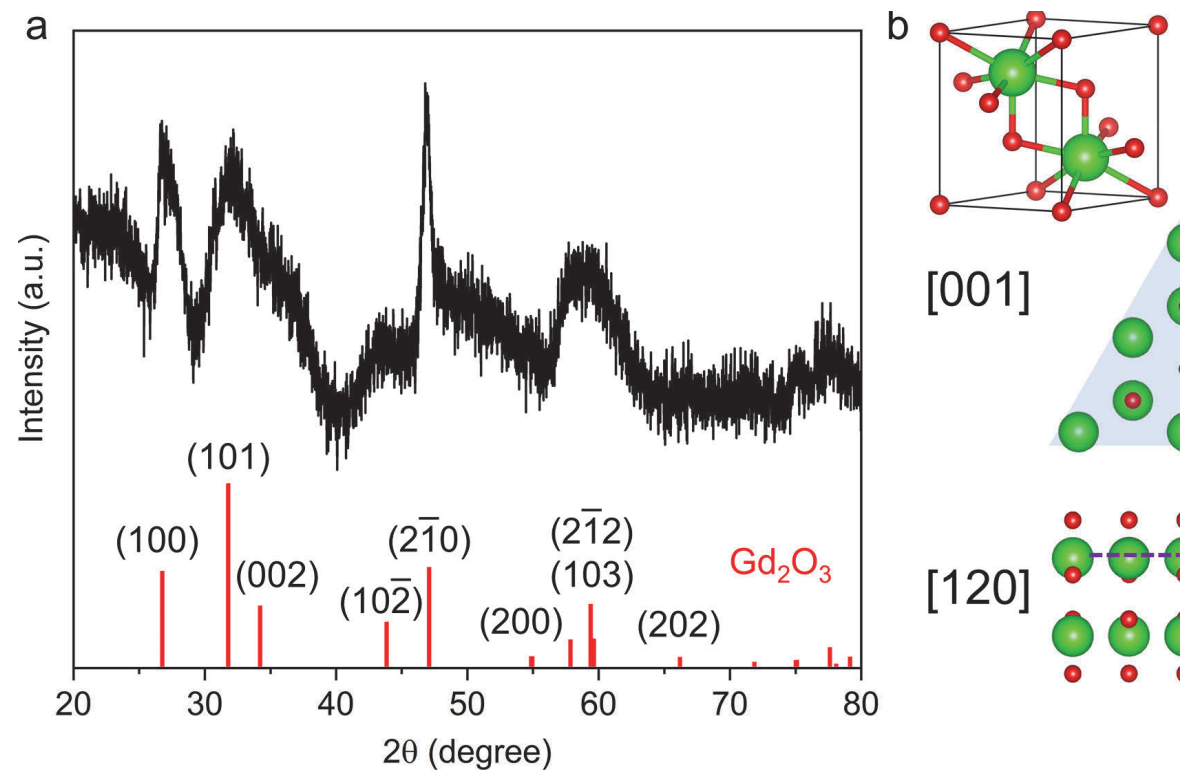

[001]

[120]

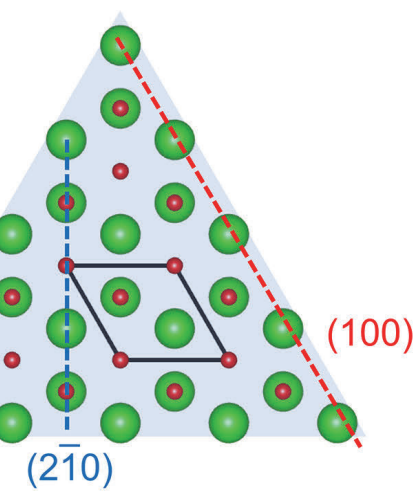

Figure S2. (a) Powder XRD pattern of as-made $\mathrm{Gd}_{2} \mathrm{O}_{3}$ triangular nanoprisms. The vertical sticks at the bottom indicate the simulated XRD pattern of hexagonal phase $\mathrm{Gd}_{2} \mathrm{O}_{3}$. (b) (upper left) Unit cell of the hexagonal phase $\mathrm{Gd}_{2} \mathrm{O}_{3}$ and crystal structure models viewed along the [001] and [120] zone axes with the unit cell indicated (black lines). The (100), (210) and (001) crystal planes are highlighted using red, blue and purple dashed lines, respectively.

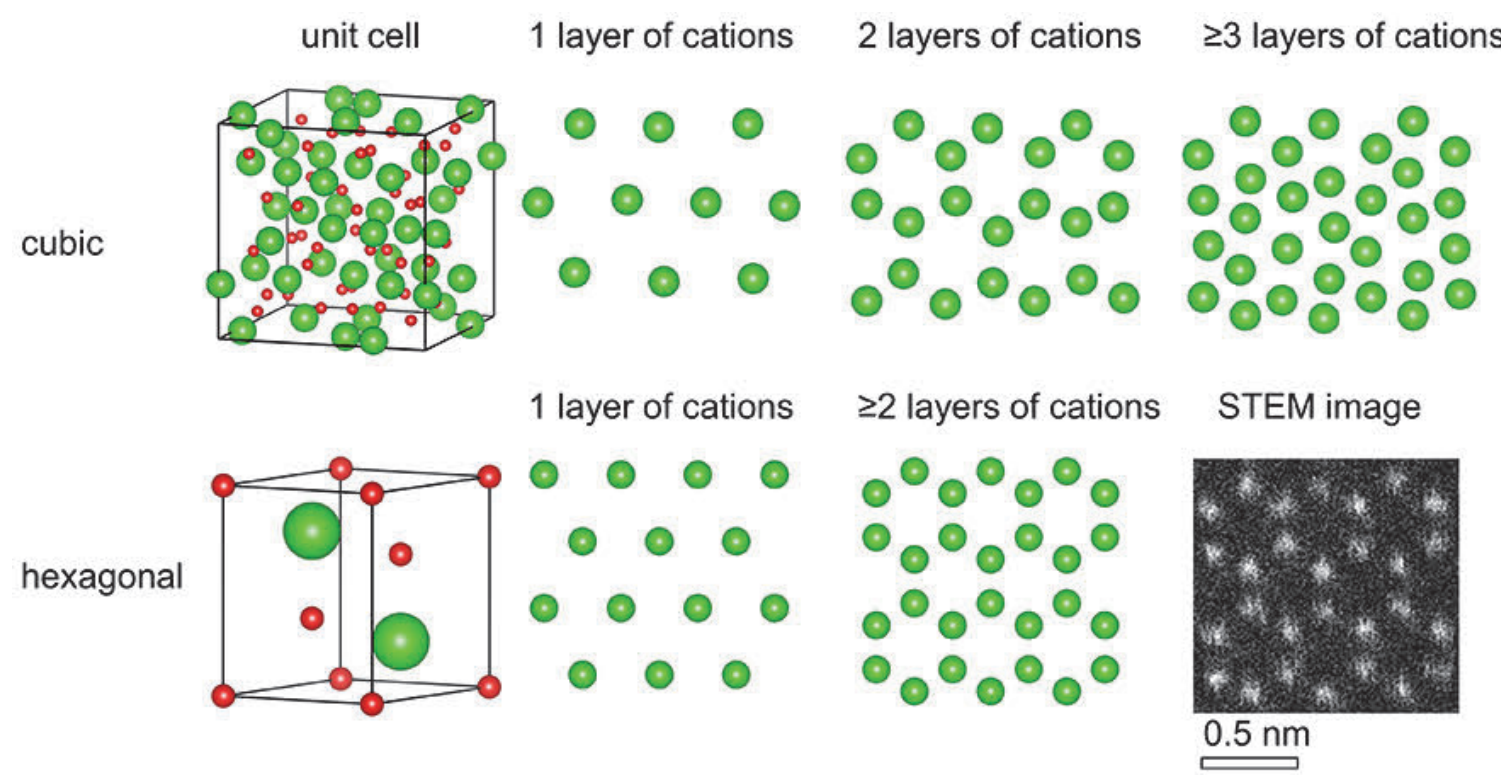

Figure S3. Comparison of crystal structures of the cubic phase and the hexagonal phase of $\mathrm{Gd}_{2} \mathrm{O}_{3}$. Unit cell and structure models viewed along the (top row) [111] and the (bottom row) [001] zone axes. Representative AC-STEM image (bottom right) acquired from a single nanoprism with electron beam orthogonal to its basal planes reveals a honeycomb arrangement of the cation lattice. Edge-view TEM images revealed that individual nanoprisms comprise of four layers of cations in thickness (not shown), which contradicts the cubic crystal structure but supports the hexagonal crystal structure. Of note, the hexagonal crystal structure was previously reported for $\mathrm{La}_{2} \mathrm{O}_{3},{ }^{65} \mathrm{Nd}_{2} \mathrm{O}_{3},{ }^{66}$ and $\mathrm{Gd}_{2} \mathrm{O}_{3} .{ }^{67}$ 

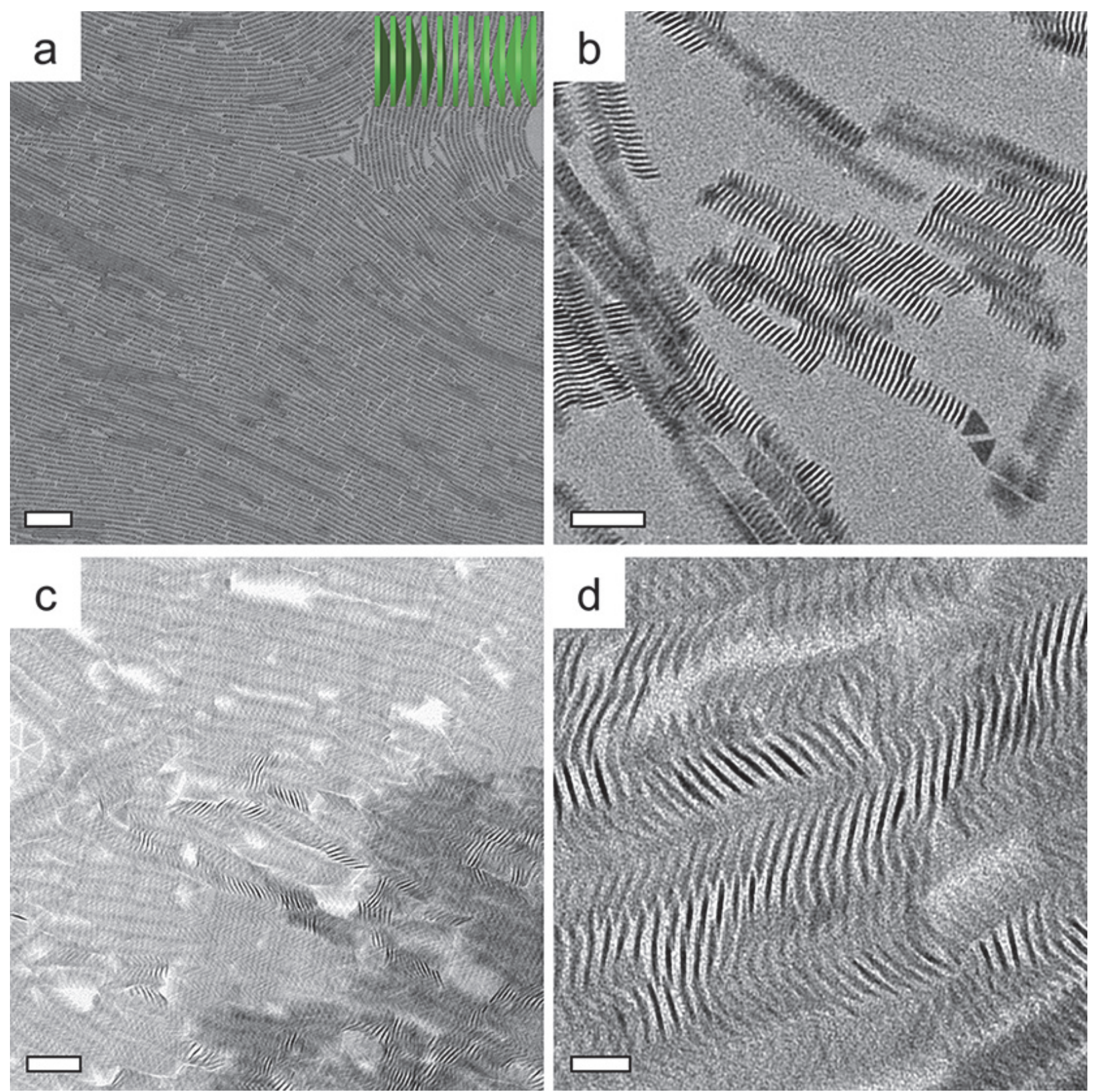

Figure S4. (a,c) Low-magnification and (b,d) high-magnification TEM images of linear stacks of as-made $\mathrm{Gd}_{2} \mathrm{O}_{3}$ triangular nanoprisms (edge length: $23.5 \mathrm{~nm}$ ) formed via self-assembly on top of an EG sub-phase (a,b) and by drop-casting of nanoprism solution onto a carbon-coated copper TEM grid. Scale bars: (a) 200 $\mathrm{nm}$, (b, c) $50 \mathrm{~nm}$, and (d) $20 \mathrm{~nm}$. 

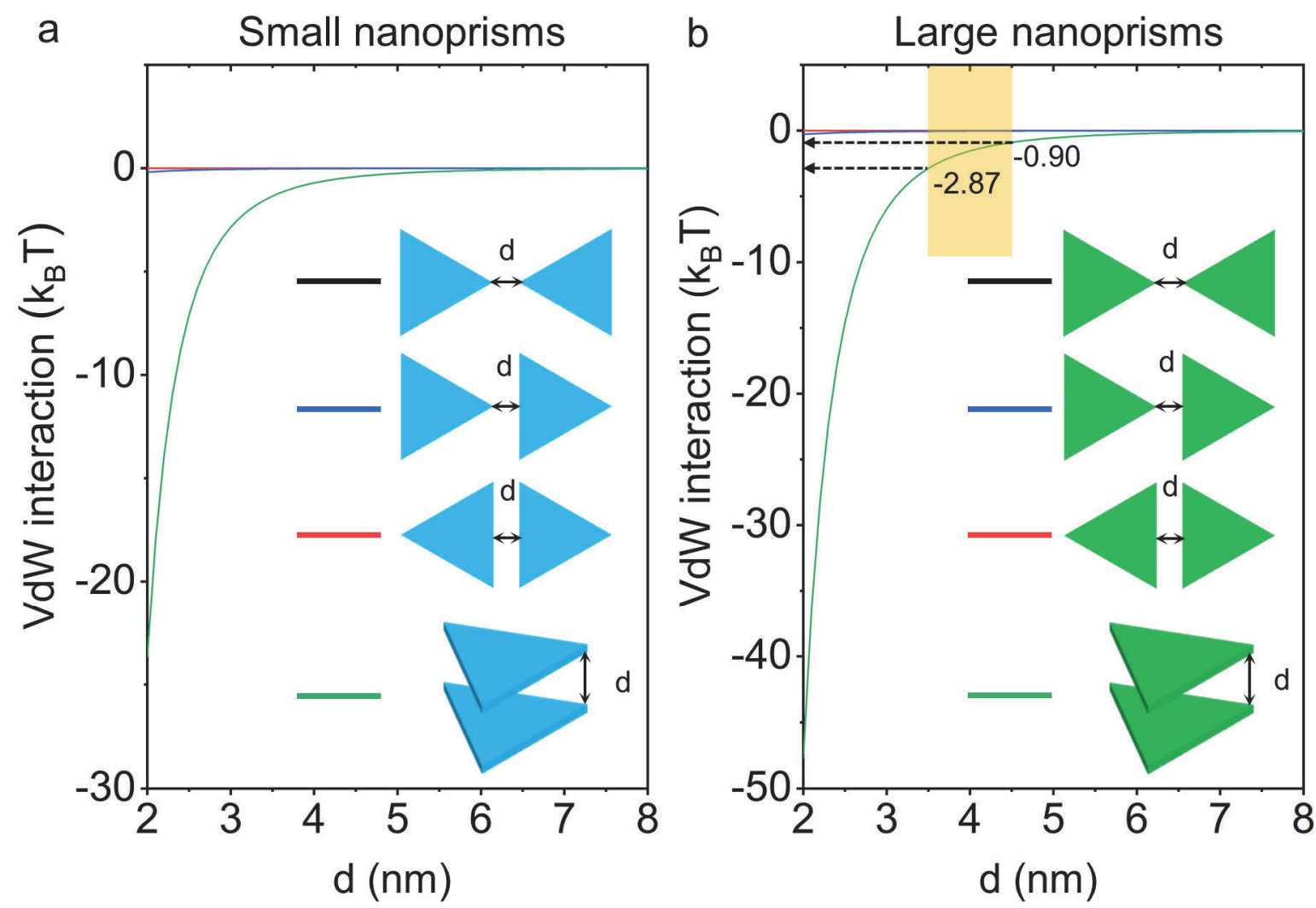

Figure S5. Distance-dependent pairwise vdW interactions calculated for (a) small (17.0-nm edge length) and (b) large (23.5-nm edge length) $\mathrm{Gd}_{2} \mathrm{O}_{3}$ triangle nanoprisms at four distinct configurations. 
a
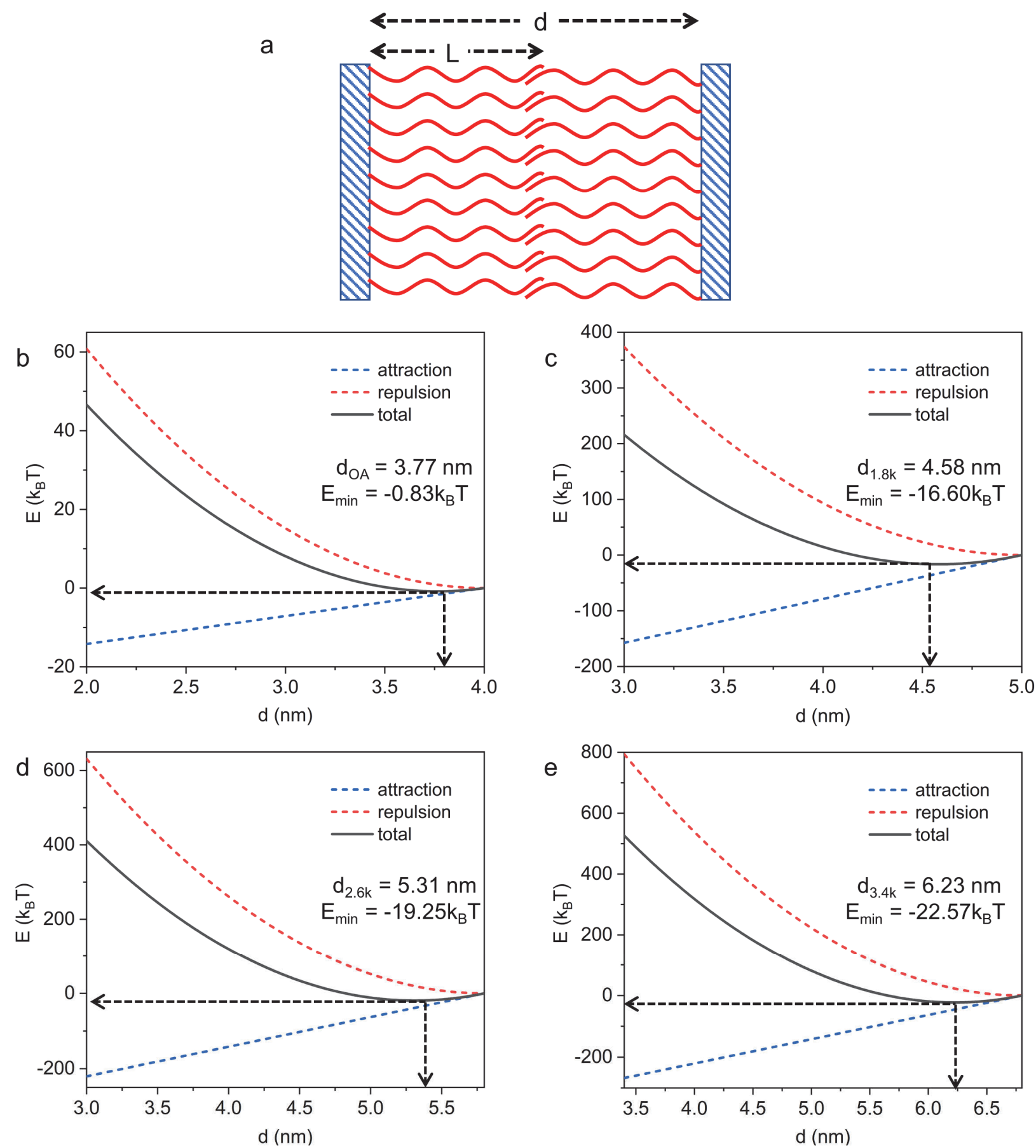

Figure S6. (a) Schematic illustration of interpenetrating polymeric ligands tethered to a pair of opposing flat surfaces. (b-e) Attractive vdW interaction energy (blue dashed line), repulsive elastic energy (red dashed line) and their sum (black line) for a pair of surface-bound OA ligands (b), $1.8 \mathrm{kDa}$ PS (c), $2.6 \mathrm{kDa}$ PS (d) and $3.4 \mathrm{kDa}$ PS at different distances. The location and depth of total energy minima are denoted using black dashed arrows. 


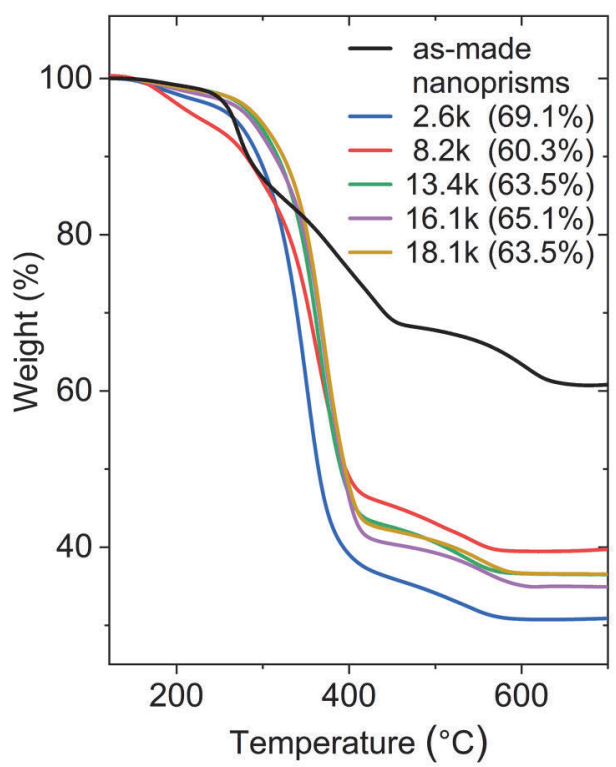

Figure S7. TGA results of as-made and PS-grafted $\mathrm{Gd}_{2} \mathrm{O}_{3}$ nanoprisms (edge length: $23.5 \mathrm{~nm}$ ) obtained using the direct ligand-exchange method.

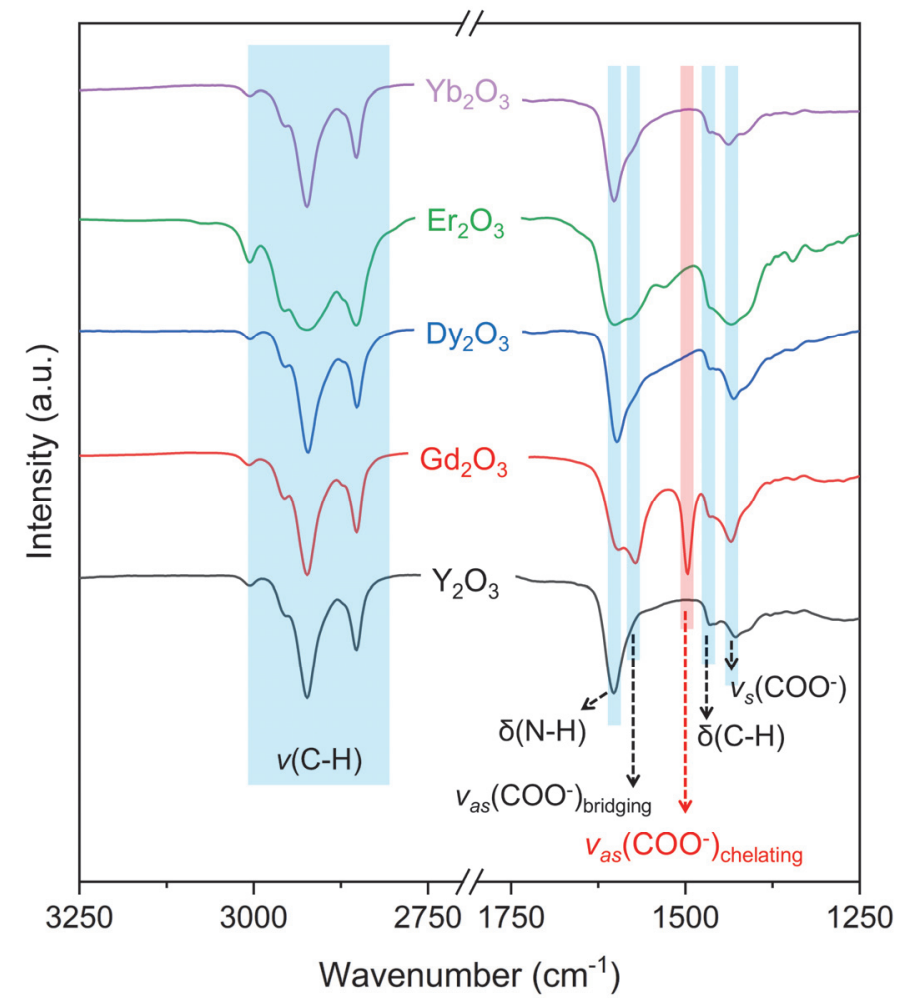

Figure S8. FTIR spectra of several rare-earth metal oxide nanocrystals synthesized using similar protocols as the $\mathrm{Gd}_{2} \mathrm{O}_{3}$ triangular nanoprisms in this work. All spectra share the following set of peaks: 3010-2800 $\mathrm{cm}^{-1}$ (C-H stretch), $1600 \mathrm{~cm}^{-1}$ (N-H bending), $1570 \mathrm{~cm}^{-1}$ (asymmetric stretching vibration of bridging O$\mathrm{C}-\mathrm{O}$ ), $1465 \mathrm{~cm}^{-1}\left(\mathrm{CH}_{2}\right.$ in-plane scissoring vibration), and $1433 \mathrm{~cm}^{-1}$ (symmetric stretch of $\mathrm{O}-\mathrm{C}-\mathrm{O}$ ). However, the FTIR spectrum of $\mathrm{Gd}_{2} \mathrm{O}_{3}$ nanoprisms showed an additional peak centered at $1496 \mathrm{~cm}^{-1}$, which we attribute to the asymmetric stretching vibration of chelating $\mathrm{O}-\mathrm{C}-\mathrm{O}$. 

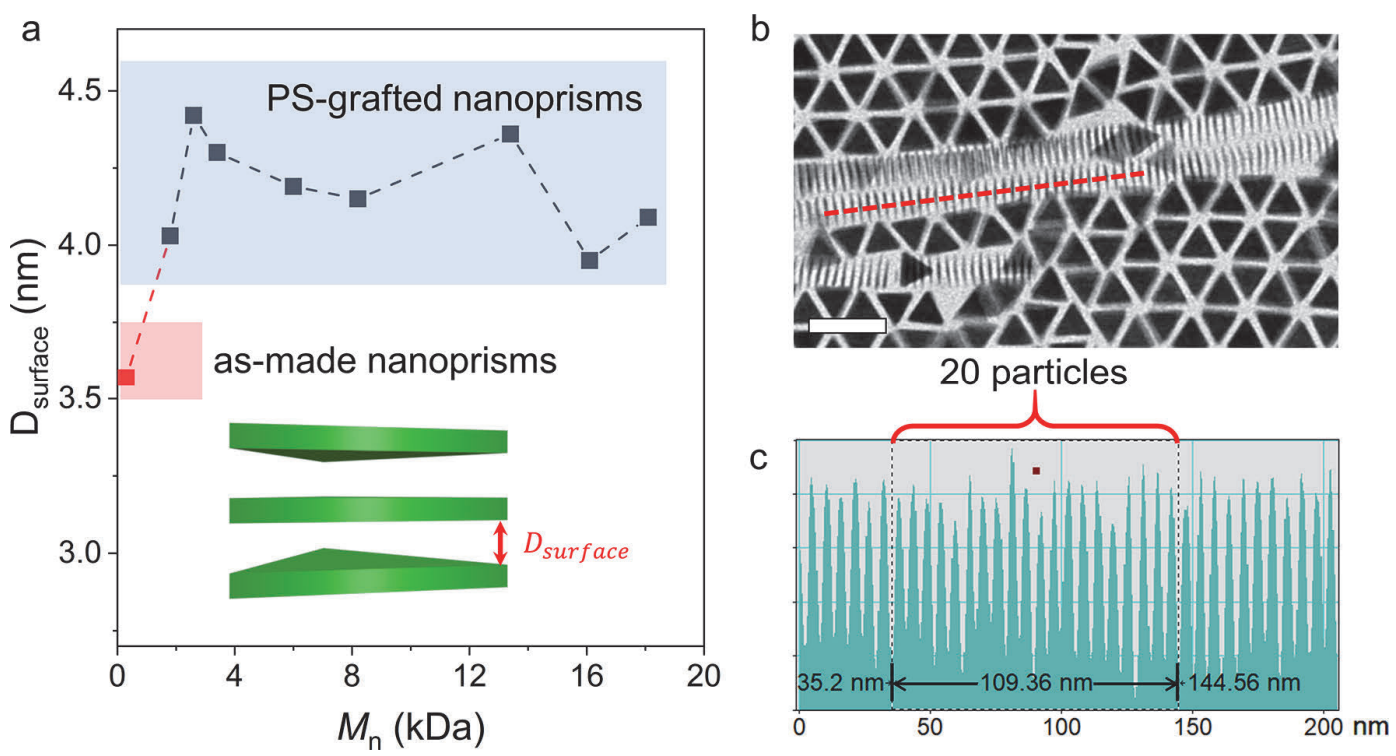

Figure S9. (a) Nearest-neighbor distances measured from vertically standing linear stacks of as-made (i.e., OA-capped and OLAM-capped) and PS-grafted $\mathrm{Gd}_{2} \mathrm{O}_{3}$ nanoprisms (edge length: $23.5 \mathrm{~nm}$ ) obtained using the direct ligand-exchange method. (b) An example of the measurement of the nearest-neighbor face-toface distance from self-assembled PS-grafted nanoprism superstructures with co-existing horizontally lying and vertically standing domains and (c) corresponding line profile along the red dashed line indicated in (b). Scale bar: $50 \mathrm{~nm}$.

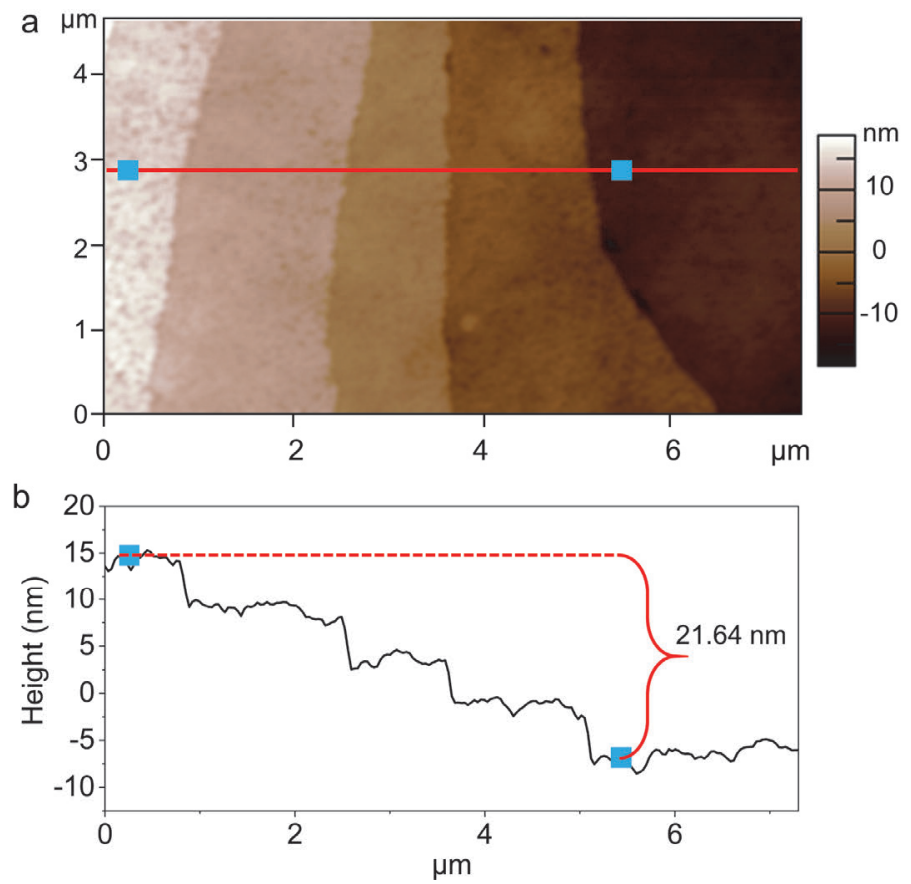

Figure S10. (a) Tapping-mode AFM image of ordered superstructures comprised of PS (8.2 kDa)-grafted $\mathrm{Gd}_{2} \mathrm{O}_{3}$ nanoprisms (edge length: $23.5 \mathrm{~nm}$ ) obtained using direct ligand-exchange method. (b) Corresponding height profile across four nanoprism layers near the edge of a superstructure domain. The average layer spacing is determined to be $5.4 \mathrm{~nm}$. 

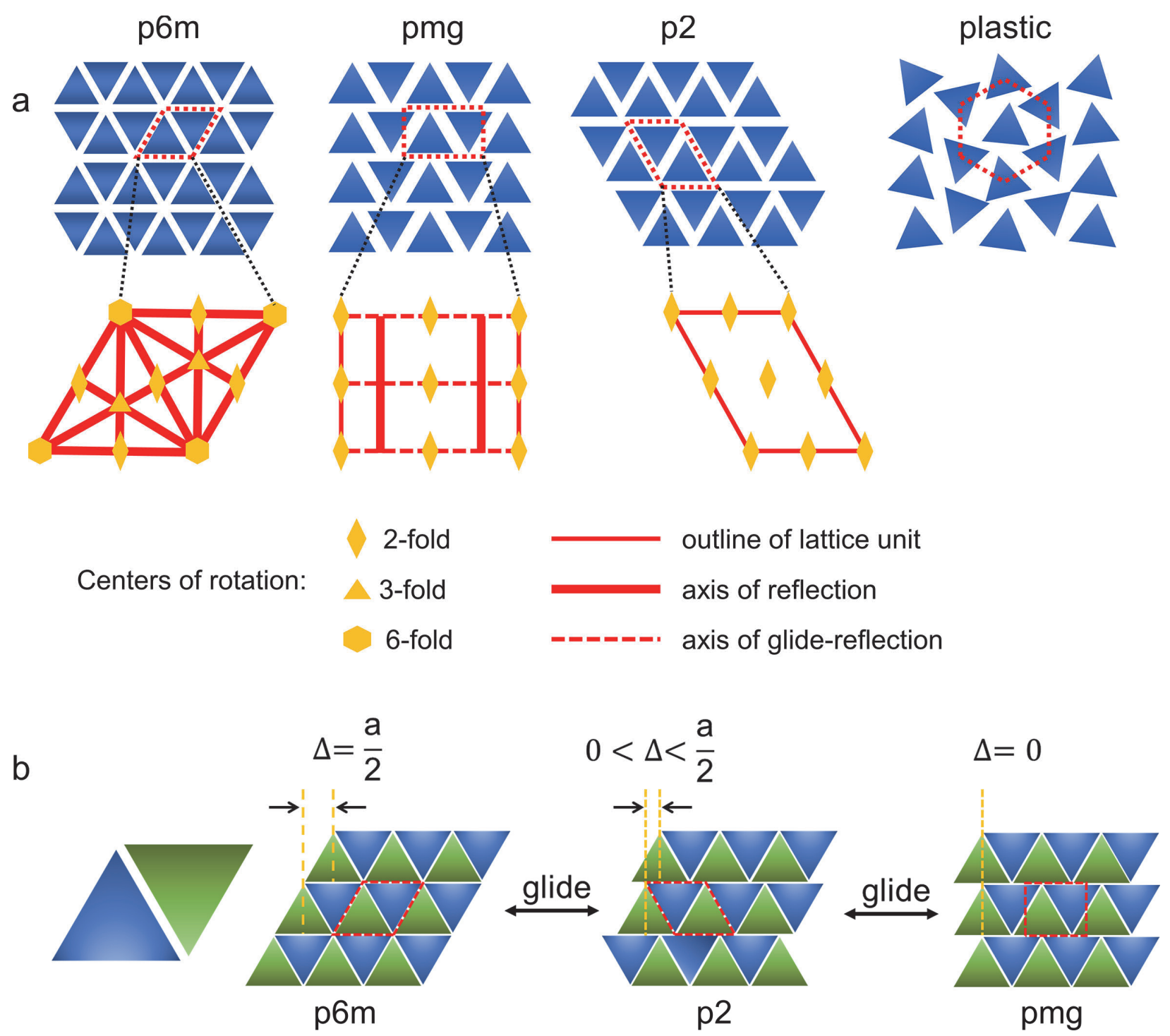

Figure S11. (a) Schematic illustration of distinct planar wallpaper symmetries using triangular prisms as tiles, as well as the unit cell and symmetry operations of each superstructure. (b) Schematic illustration of the structural relationship of p6m-type, p2-type, and pmg-type superstructures. 


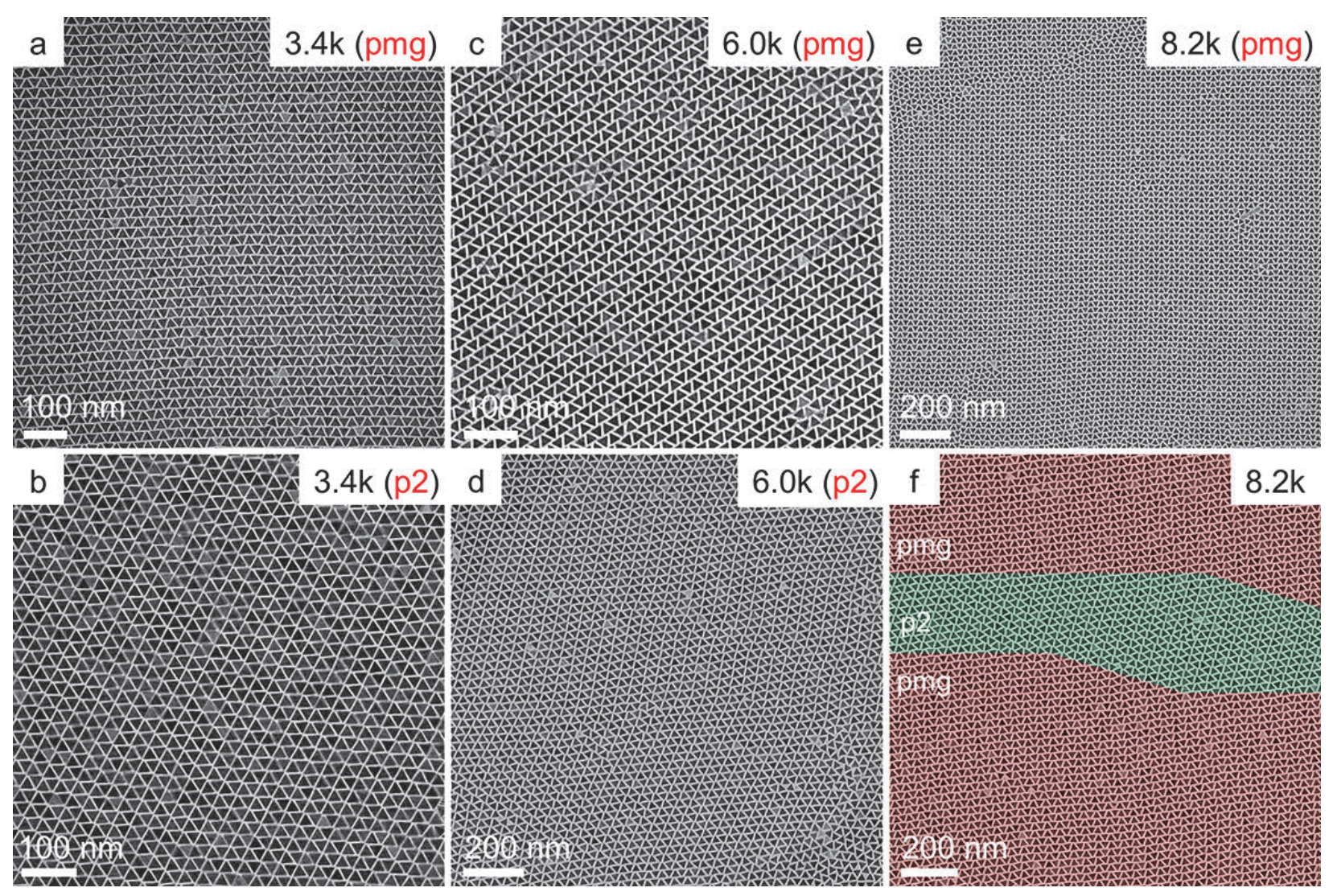

Figure S12. (a-e) Low-magnification TEM images of ordered superstructures self-assembled from PSgrafted $\mathrm{Gd}_{2} \mathrm{O}_{3}$ nanoprisms (edge length: $23.5 \mathrm{~nm}$ ) with different ligand length prepared by using the direct ligand-exchange method. (f) TEM image of co-existing pmg-type (majority phase) and p2-type (minority phase) ordered superstructures comprised of $8.2 \mathrm{kDa}$ PS-grafted nanoprisms. 

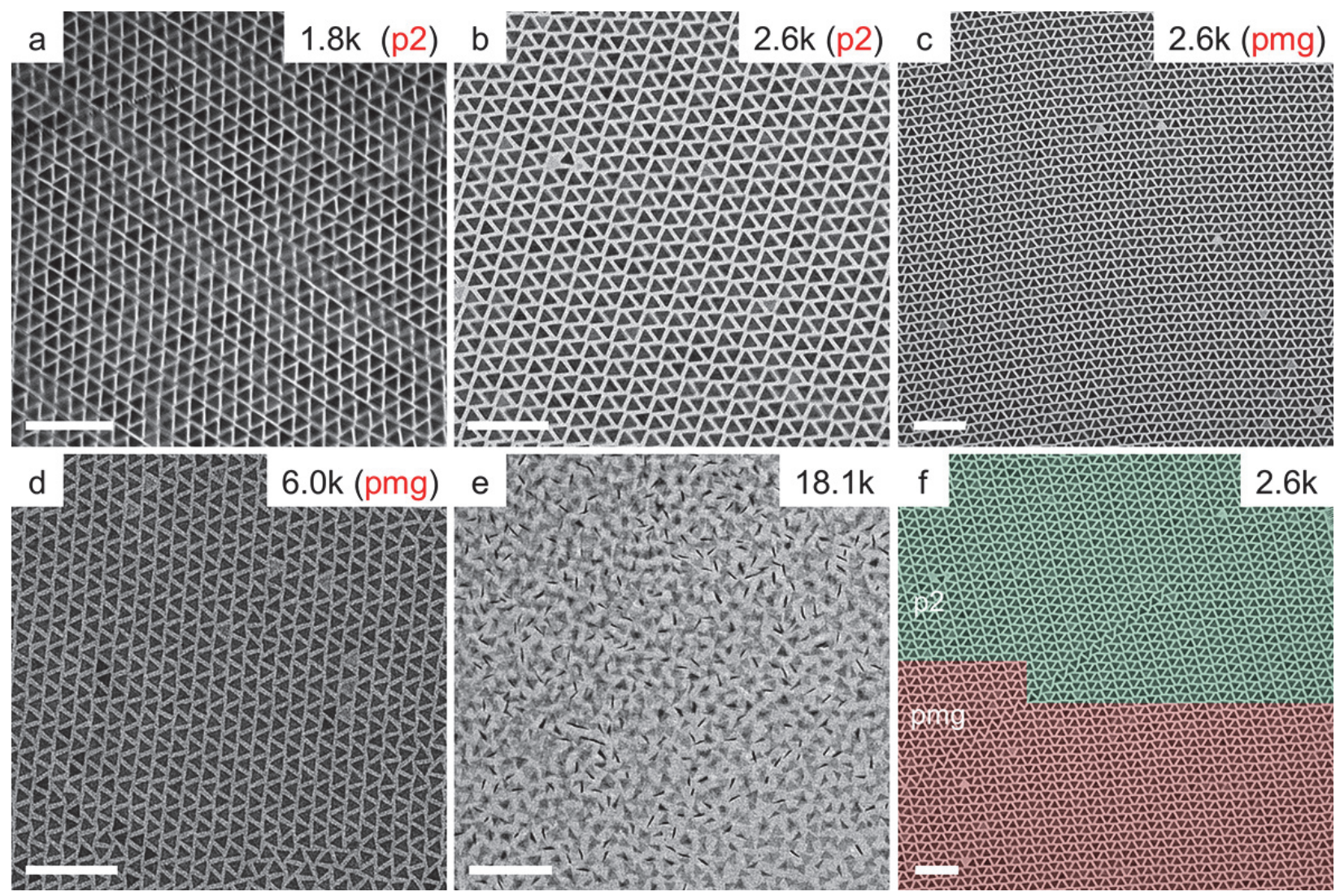

Figure S13. (a-e) TEM images of superstructures self-assembled from PS-grafted $\mathrm{Gd}_{2} \mathrm{O}_{3}$ nanoprisms (edge length: $17.0 \mathrm{~nm}$ ) with different ligand length prepared by using the direct ligand-exchange method. (f) TEM image of co-existing pmg-type and p2-type ordered superstructures comprised of $2.6 \mathrm{kDa}$ PS-grafted nanoprisms. Scale bars: $100 \mathrm{~nm}$. 


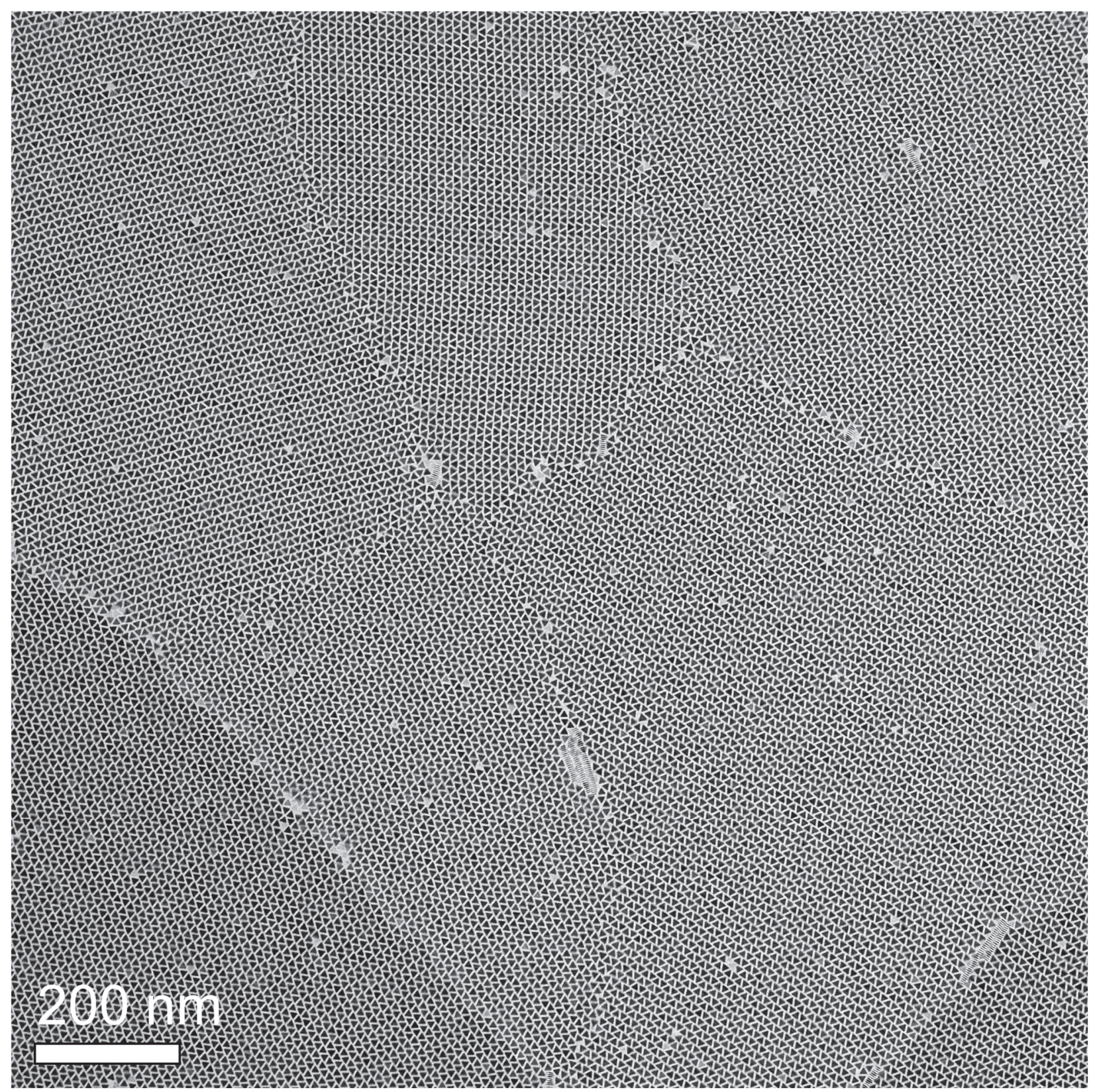

Figure S14. Low-magnification TEM image of pmg-type superstructures self-assembled from PS (2.6 $\mathrm{kDa}$ )-grafted $\mathrm{Gd}_{2} \mathrm{O}_{3}$ nanoprisms (edge length: $17.0 \mathrm{~nm}$ ) prepared by using the direct ligand-exchange method. 

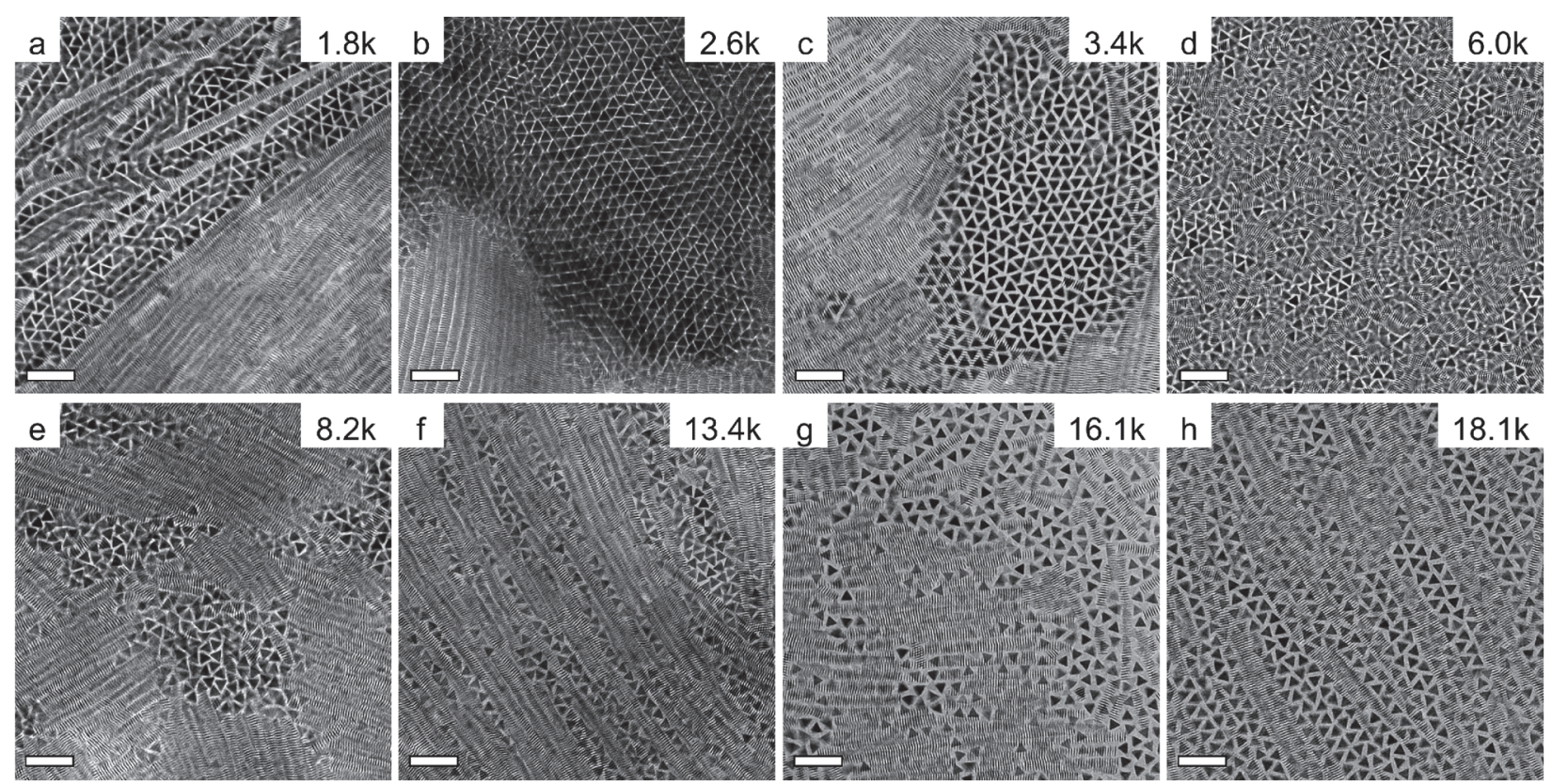

Figure S15. Representative TEM images of assemblies of PS-grafted $\mathrm{Gd}_{2} \mathrm{O}_{3}$ nanoprisms (edge length: 23.5 $\mathrm{nm}$ ) made with the direct ligand-exchange method using suboptimal feeding grafting densities of PS-PEHA ligands. The feeding grafting densities of PS-PEHA are: (a) $9.0 \mathrm{~nm}^{-2}$, (b) $9.2 \mathrm{~nm}^{-2}$, (c) $9.0 \mathrm{~nm}^{-2}$, (d) $4.1 \mathrm{~nm}^{-}$ , (e) $2.1 \mathrm{~nm}^{-2}$, (f) $2.1 \mathrm{~nm}^{-2}$, (g) $2.1 \mathrm{~nm}^{-2}$ and (h) $1.5 \mathrm{~nm}^{-2}$. Scale bars: $100 \mathrm{~nm}$.
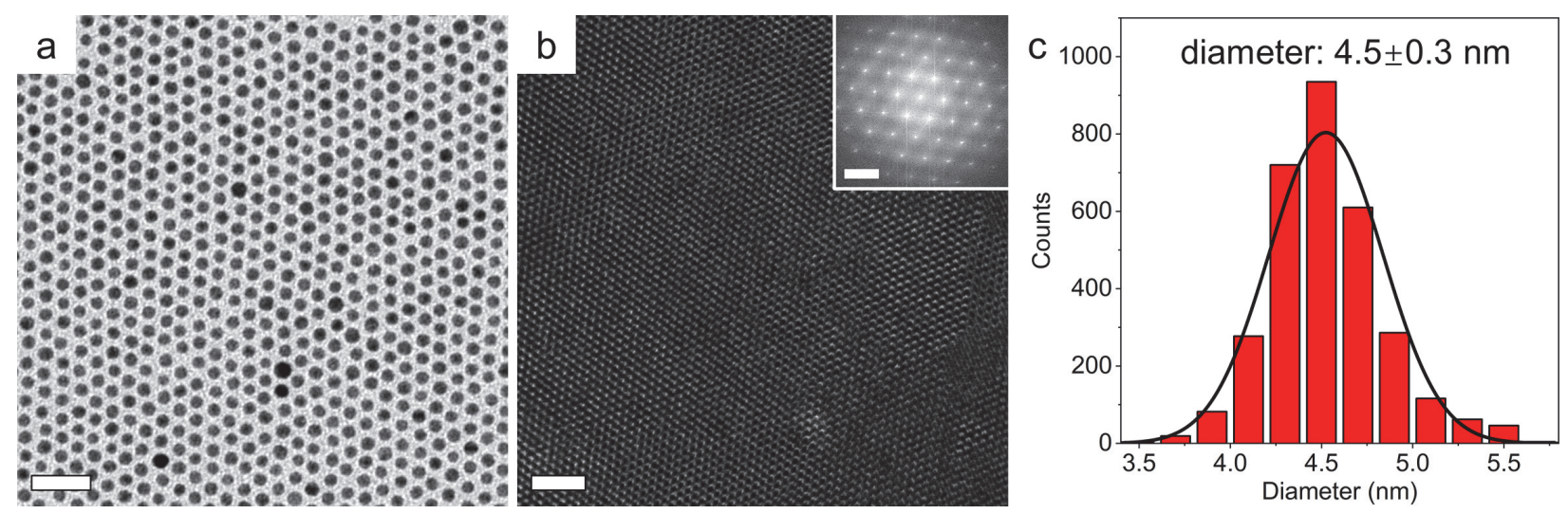

Figure S16. TEM images of (a) two-dimensional and (b) three-dimensional assemblies of OLAM-capped Au nanocrystals and (c) size distribution histogram. The inset of panel (b) is the corresponding FFT pattern. Scale bars: (a) $20 \mathrm{~nm}$, (b) $50 \mathrm{~nm}$, and inset of (b) $2 \mathrm{~nm}^{-1}$. 

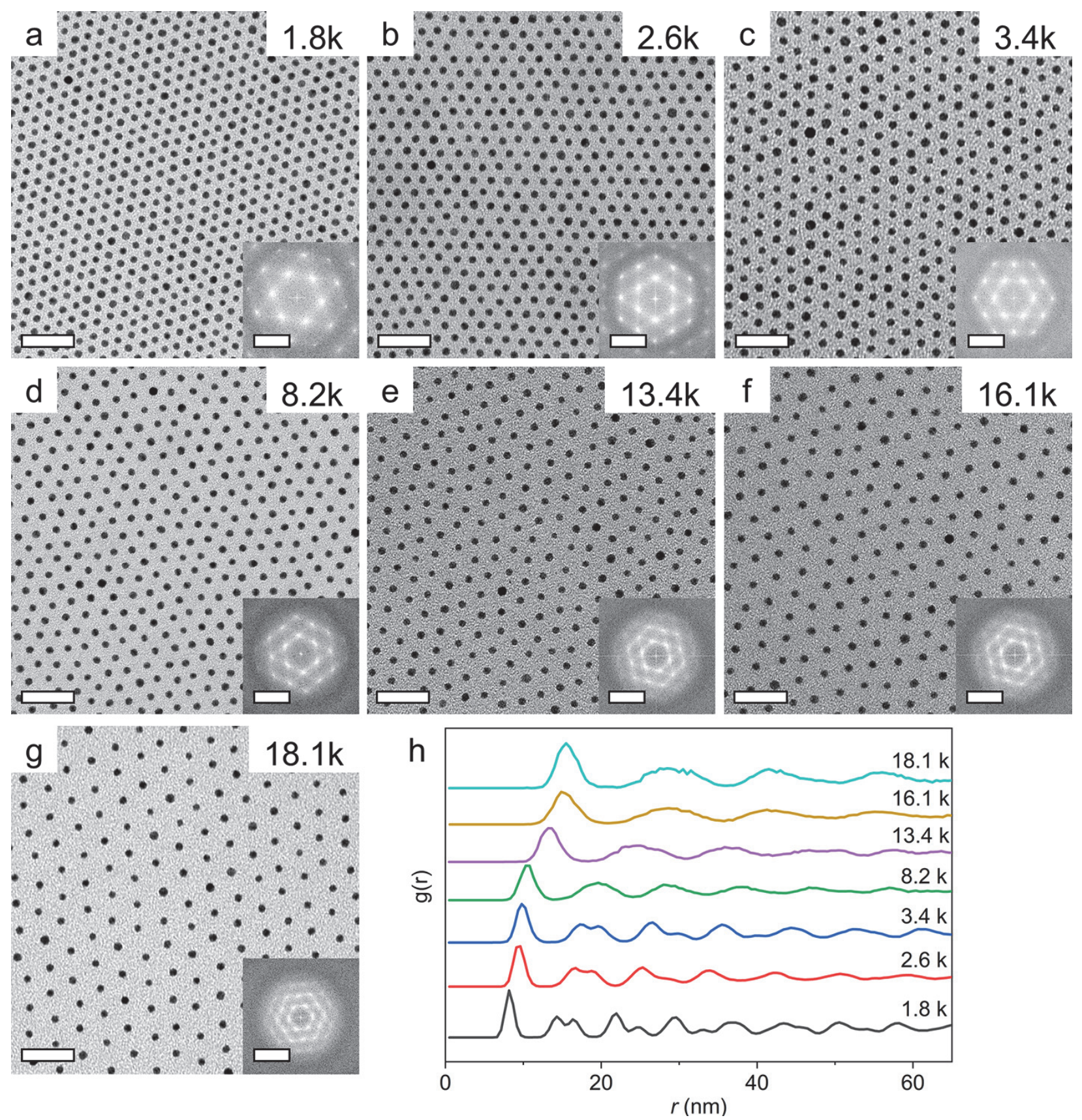

Figure S17. (a-g) Representative TEM images, corresponding FFT patterns (insets), and calculated (g) radial distribution functions of two-dimensional assemblies of 4.5-nm Au nanocrystals grafted with PSPEHA ligands of different $M_{n}$. Scale bars: $30 \mathrm{~nm}$ (main image), $0.2 \mathrm{~nm}^{-1}$ (insets). 


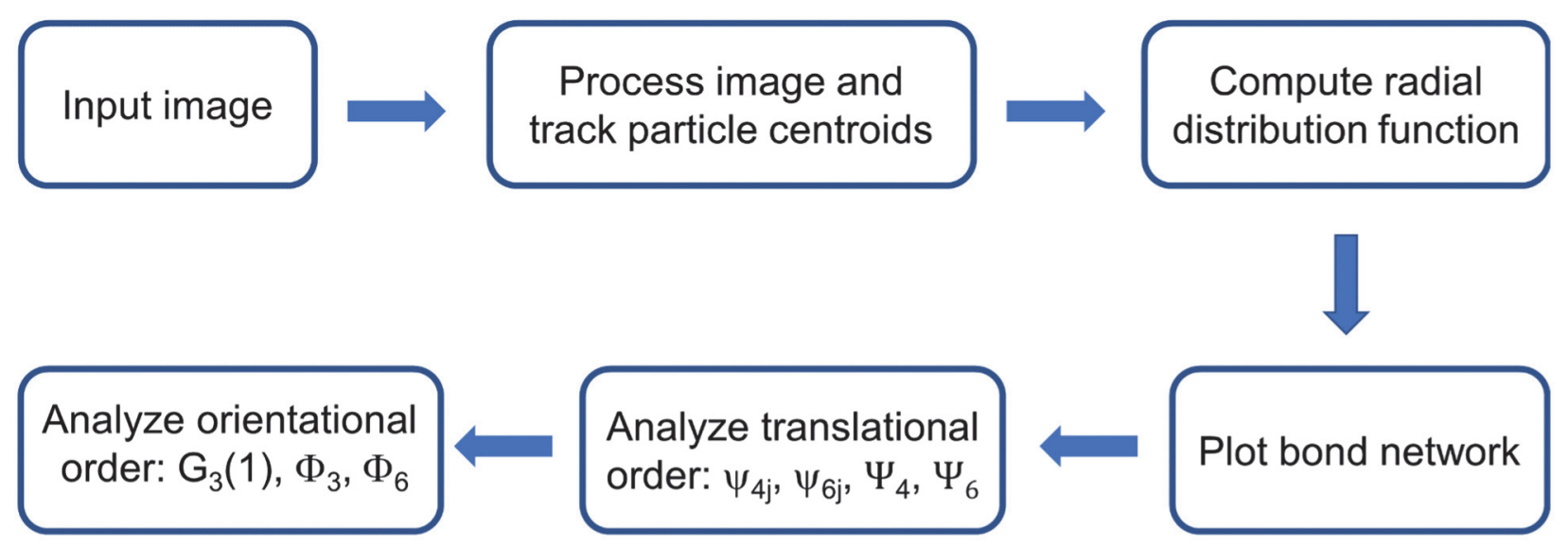

Figure S18. Flowchart of general image processing and analysis procedure.

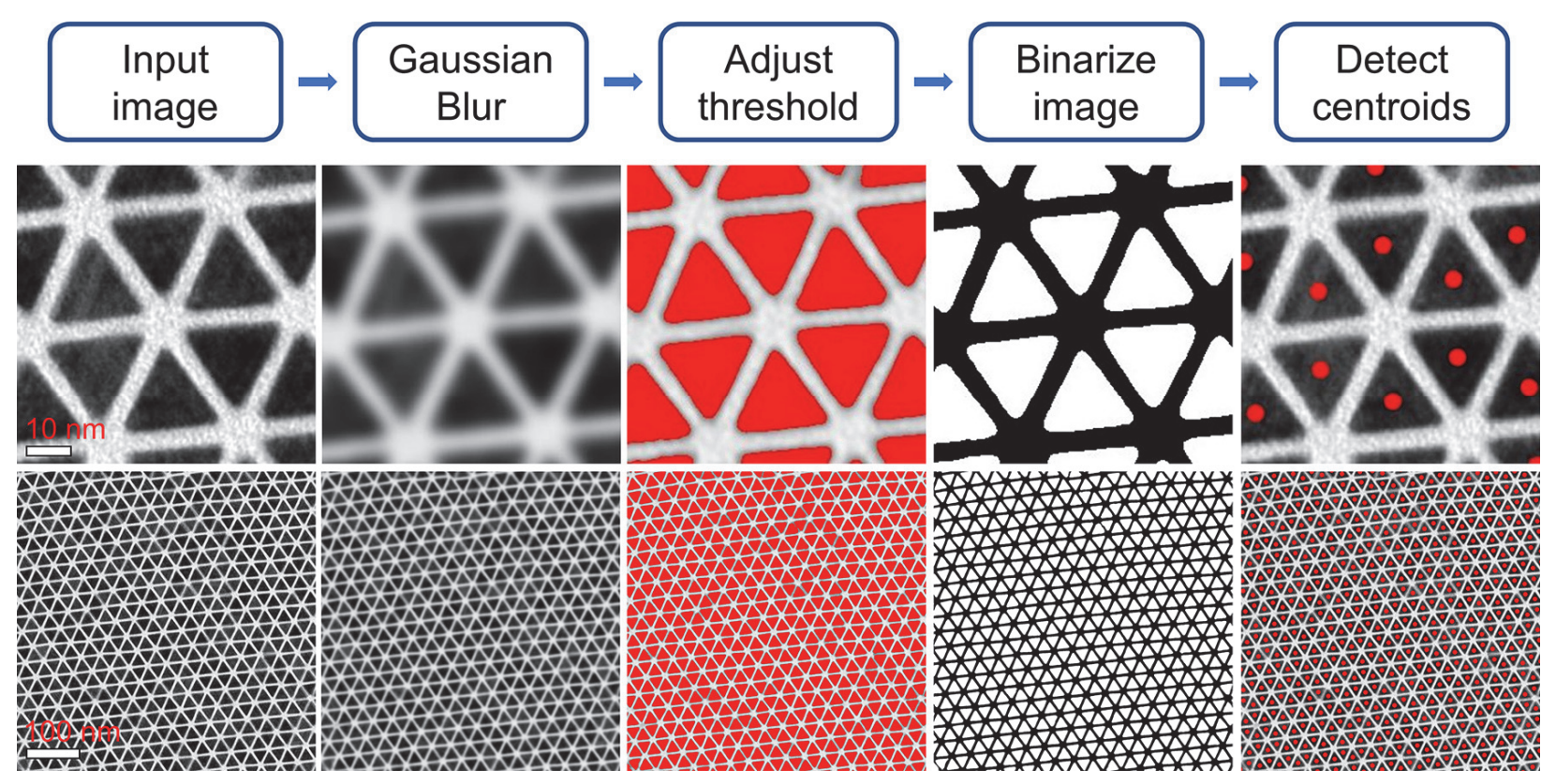

Figure S19. Flowchart and exemplary data illustrating the particle contour-finding and centroid-detection process. 

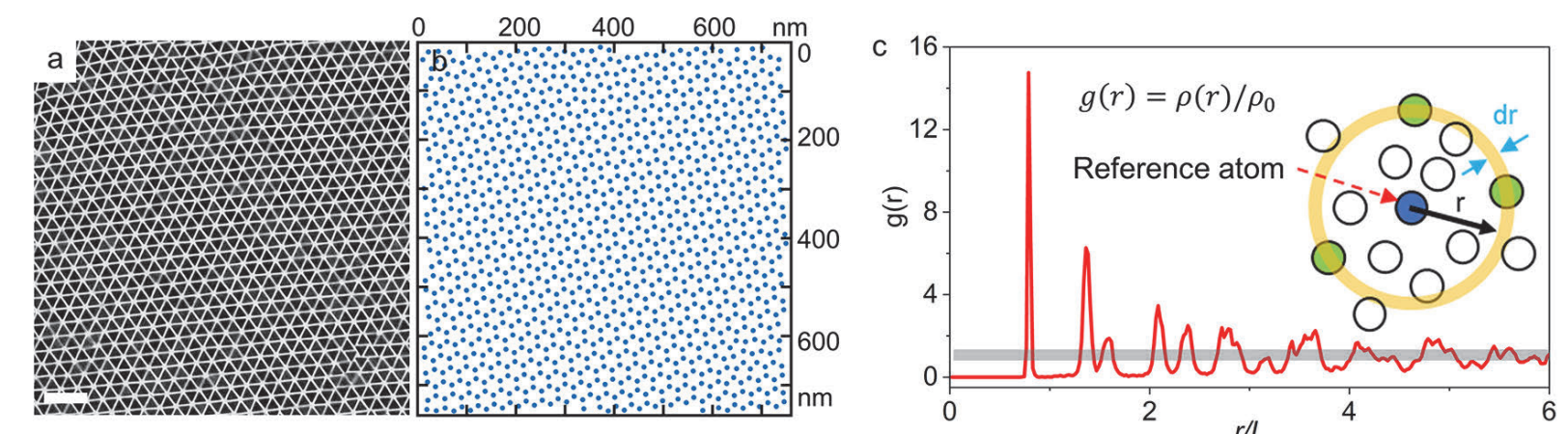

$1^{\text {st }}$ nearest neighbors

$2^{\text {nd }}$ nearest neighbors

$3^{\text {rd }}$ nearest neighbors
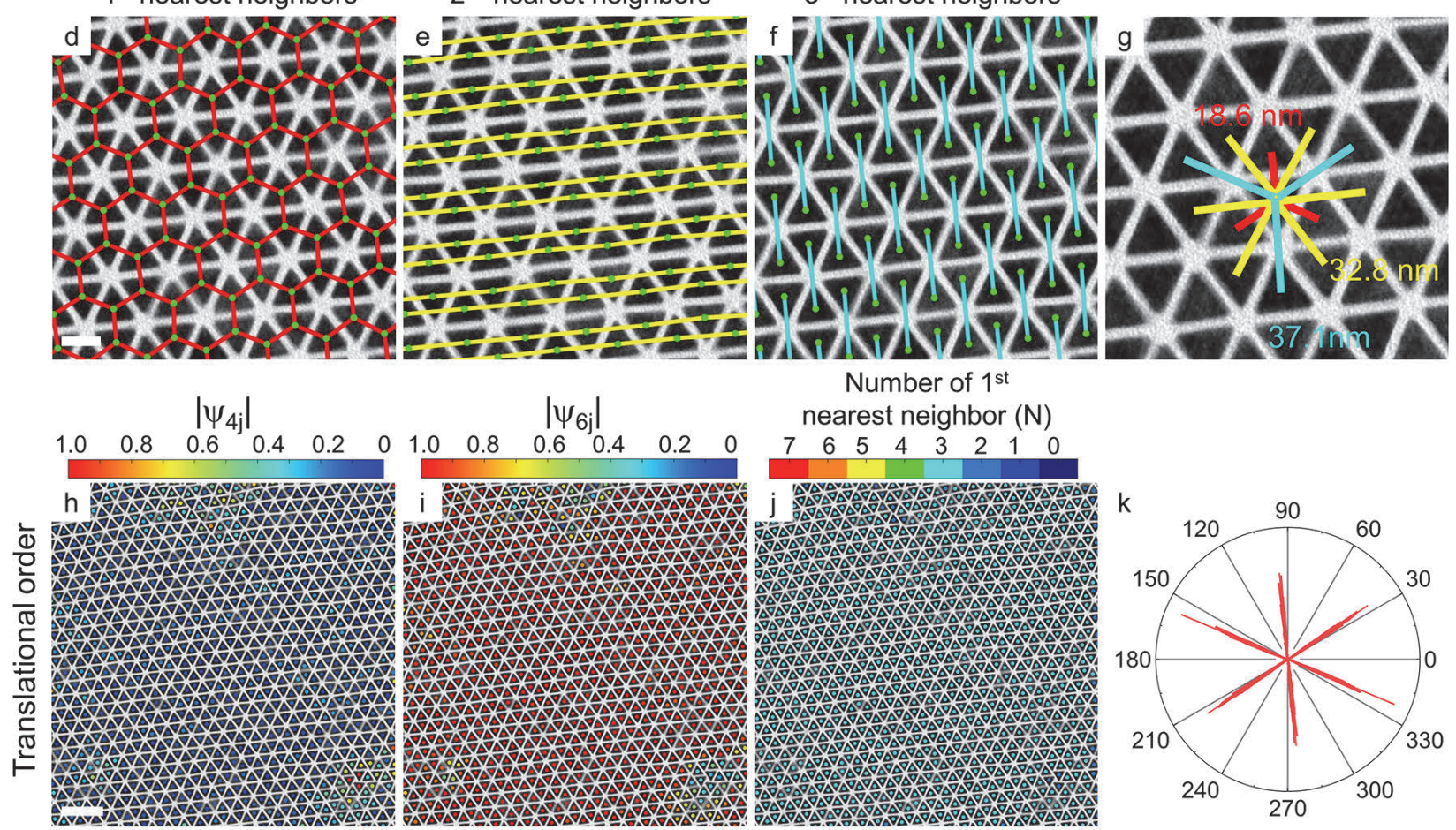

Number of $1^{\text {st }}$

nearest neighbor $(\mathrm{N})$

Angle (degree)
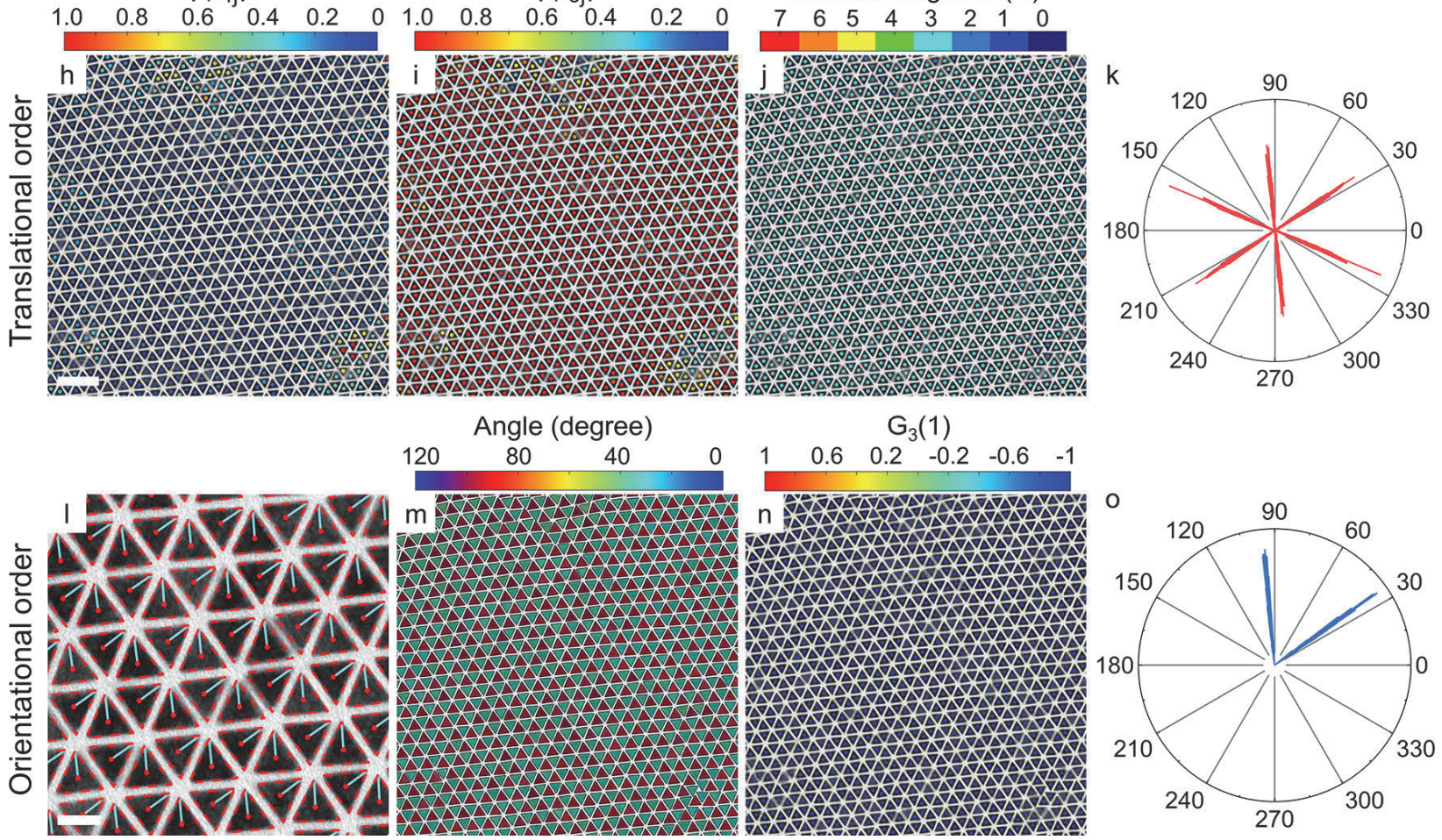

Figure S20. Analysis of translational and orientational order of the p6m-type superstructure. (a) Original TEM image and (b) plot of triangle centroids obtained from image-analysis procedure outlined in Figure S19. (c) Radial distribution function $g(r)$ computed based on the positions of particle centroids. (d-f) TEM images and overlaid bond networks of the first (d), the second (e) and the third (f) nearest neighbors for the p6m-type superstructures self-assembled from PS-grafted nanoprisms (edge length: $23.5 \mathrm{~nm}$ ). Notably, two thirds of the bonds in (e) and (f) are omitted for clarity. (g) Plots of the distribution of the first three nearest 
neighbors around a reference particle. (h-i) TEM images color-coded according to the modulus of four-fold and six-fold local bond orientational order parameters $\left|\psi_{4 j}\right|$ and $\left|\psi_{6 j}\right|$, respectively. The global bond orientational order was determined to be $\Psi_{4}=0.009$ and $\Psi_{6}=0.892$. (j) TEM image color-coded according to the number of first nearest neighbors. (k) Polar histogram of bond orientation between nearest neighbors. (l) Contour-finding (red dashed lines) and determination of the orientation of individual triangles defined as the angle between the cyan line and the horizontal $x$-axis. (m) TEM image color-coded according to the orientation of individual triangles. (n) TEM image color-coded according to the three-fold orientational correlation function $G_{3}(1)$ of individual triangles which shows the perfect antiparallel alignment between nearest neighbors for the p6m-type superstructure. (o) Polar histogram of triangle orientations. The three-fold and six-fold global orientational order parameters of the p6m-type superstructure were determined to be $\Phi_{4}=0.001$ and $\Phi_{6}=0.976$. Scale bars: (a) $100 \mathrm{~nm}$, (d) $20 \mathrm{~nm}$, (h) $100 \mathrm{~nm}$, (l) $20 \mathrm{~nm}$. 


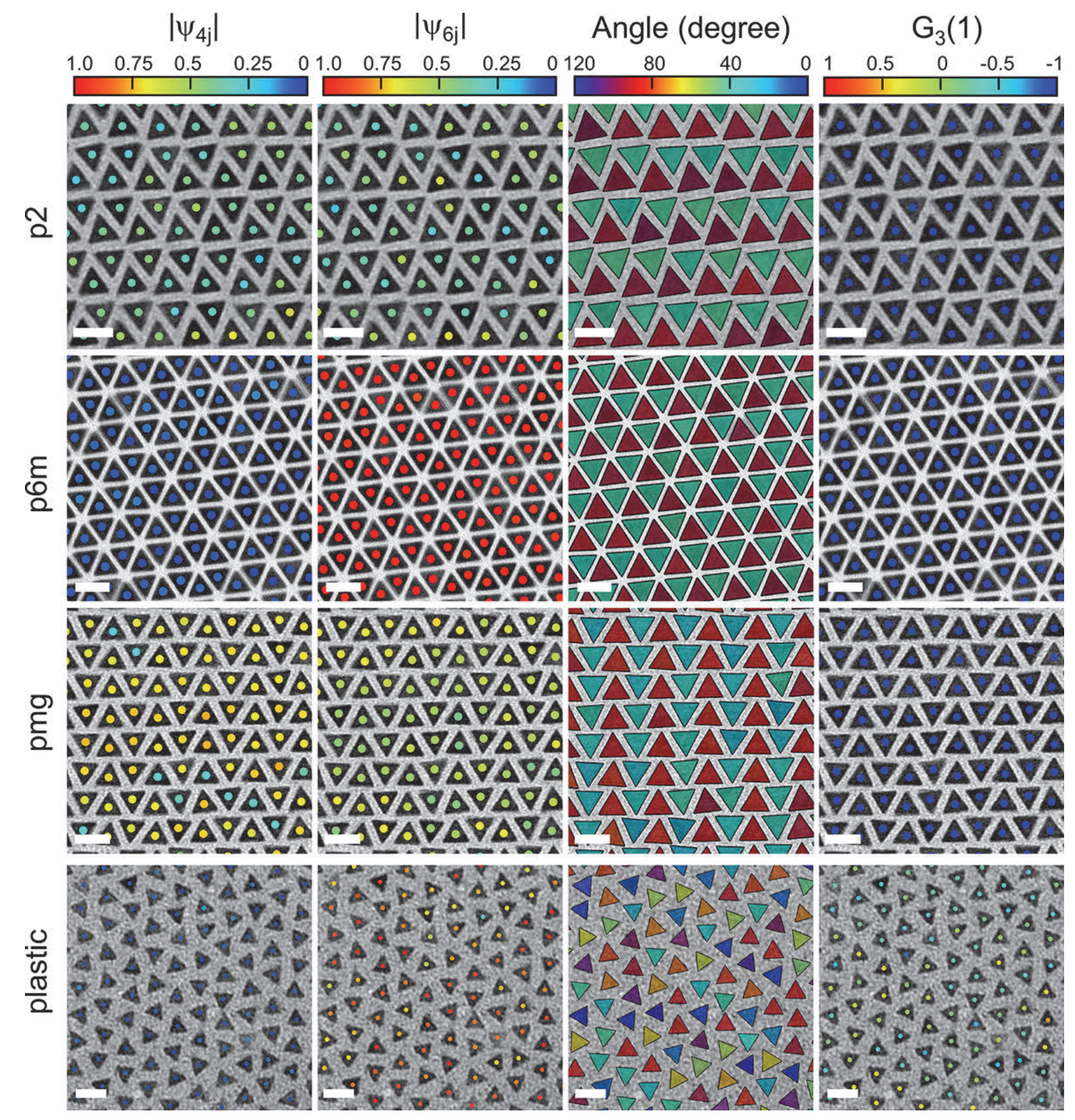

Figure S21. Analysis results of translational and orientational order of different nanoprism superstructures. The first and the second columns show TEM images color-coded according to the modulus of four-fold and six-fold local bond orientational order parameters $\left|\psi_{4 j}\right|$ and $\left|\psi_{6 j}\right|$, respectively. TEM images in the third column are color-coded based on the angle $\theta_{j}$ of individual triangles, which is defined as the angle between the horizontal $x$-axis and the line connecting the triangle center to one of its vertices $\left(0^{\circ} \leq \theta_{j} \leq 120^{\circ}\right)$. TEM images in the fourth column are color-coded according to the three-fold orientational correlation function $G_{3}(1)$ of individual triangles. Scale bars: $30 \mathrm{~nm}$. 

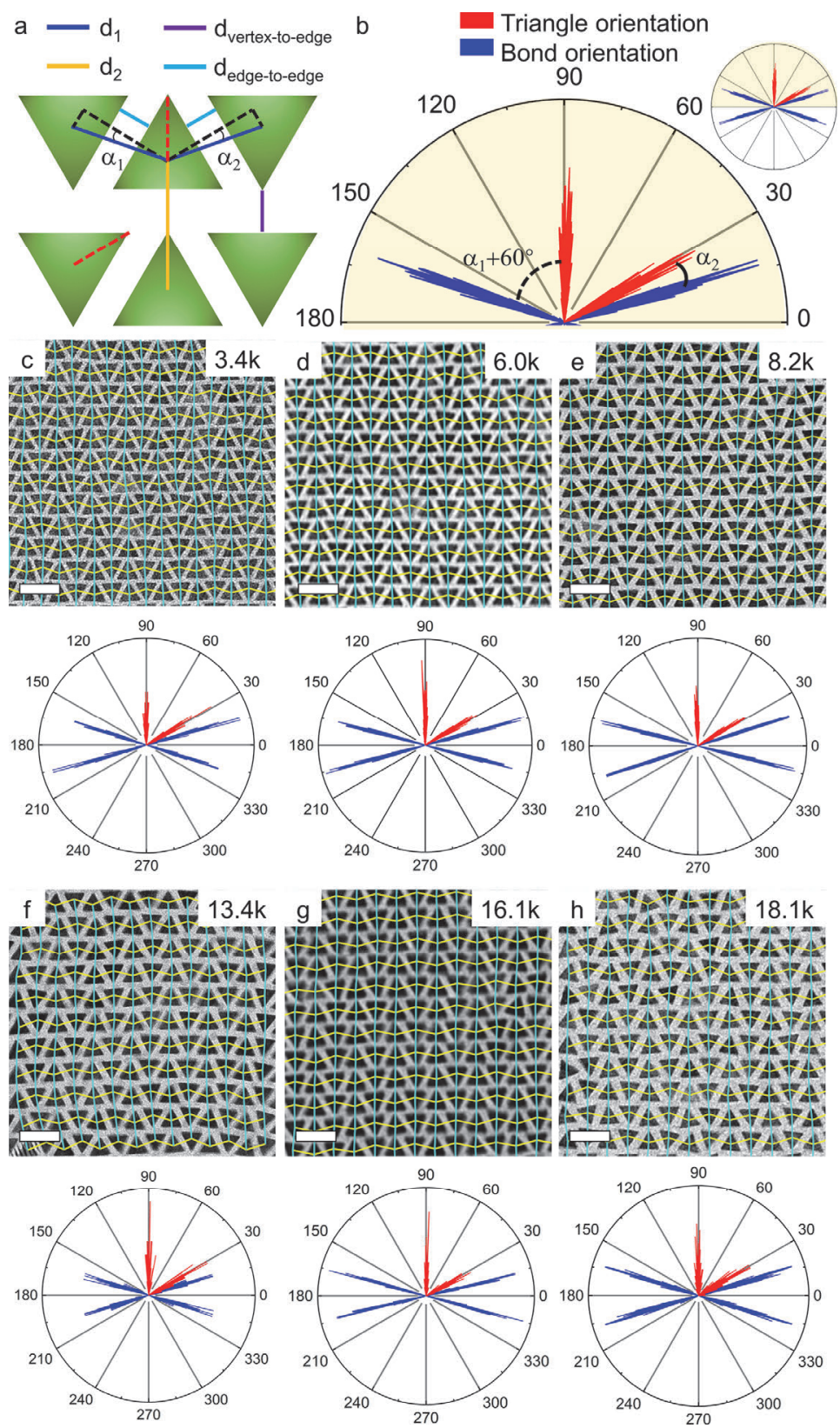

Figure S22. (a) Definition of geometric parameters for the pmg-type superstructure. (b) Exemplary polar histograms of bond orientation as well as orientation of individual triangles $d_{\text {edge-to-edge }}=d_{1} \cos (\alpha)-$ $\sqrt{3} / 3 \mathrm{~L}$ and $d_{\text {vertex-to-edge }}=d_{2}-\sqrt{3} / 2 L$, where $d_{1}$ and $d_{2}$ are the bond lengths of first and second nearest neighbors derived from radial distribution function $g(r) . L$ is the edge length of nanoprisms, and $\alpha$ is the average of angles $\alpha_{1}$ and $\alpha_{2}$, which were derived from the bond angles and triangle orientational angles. The calculated distances are shown in Figure 2m. (c-h) TEM images and overlaid bond networks for pmg-type superstructures self-assembled from PS-grafted nanoprisms (edge length: $23.5 \mathrm{~nm}$ ). The nanoprism building blocks were prepared through direct ligand-exchange with PS-PEHA of different $M_{n}$ as indicated on the images. The bonds between first and second nearest neighbors are colored in yellow and cyan, respectively. Below each TEM image are the corresponding polar histograms of bond orientation (blue) and triangle orientation (red). Scale bars: $50 \mathrm{~nm}$. 

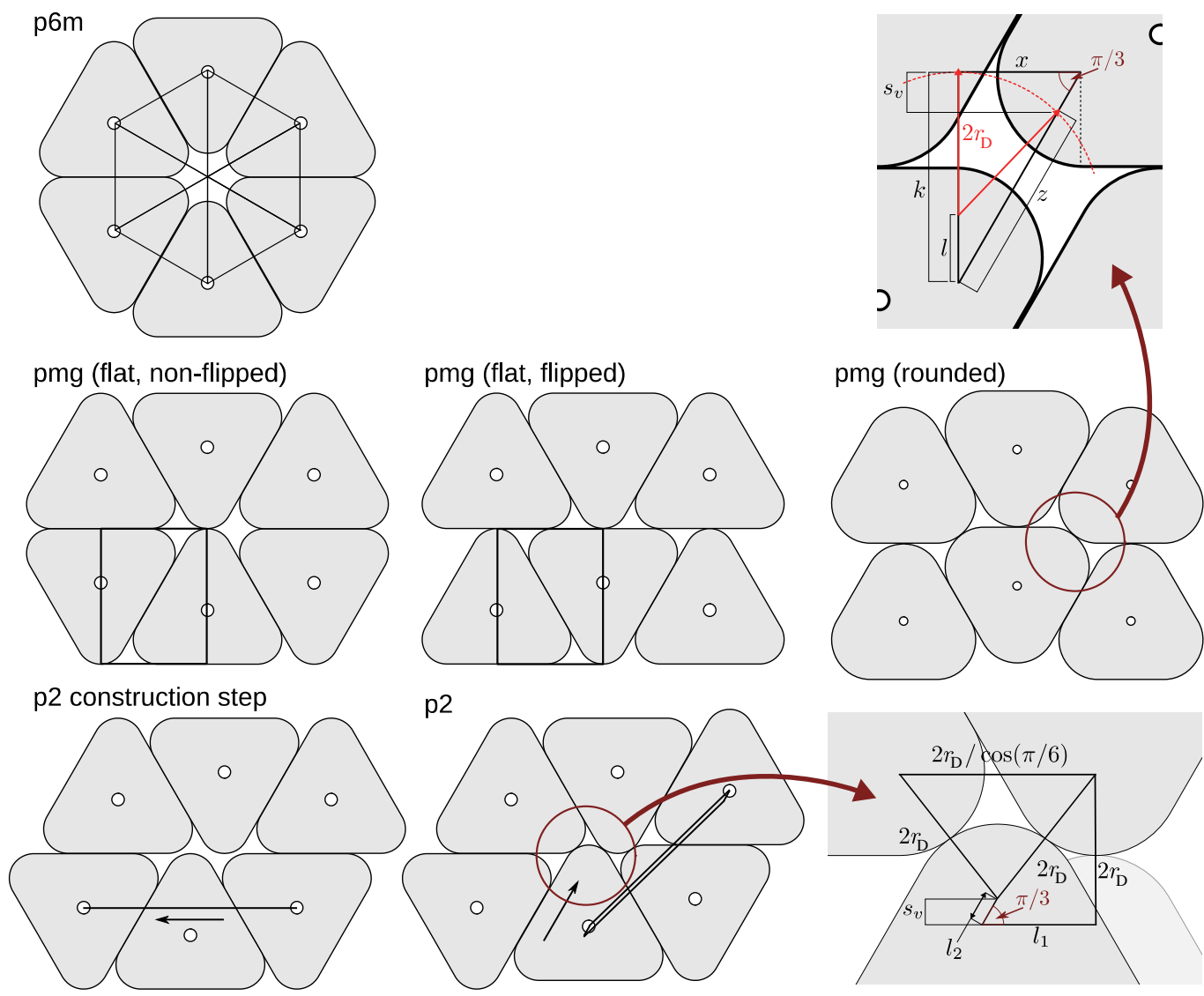

Figure S23. Geometric constructions used in the analytic densest packing calculations. The spherotriangle centers in the $\mathrm{p} 6 \mathrm{~m}$ phase forms regular hexagons. The calculation of pmg and $\mathrm{p} 2$ starts from a parallelogram. The structure can be compressed further by including small lattice shifts. The densest packing of pmg (rounded) and p2 is calculated as deviation from pmg (flat) due to small lattice shifts.

p6m

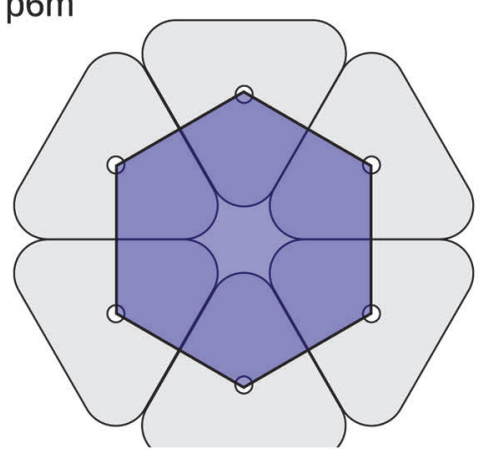

p2

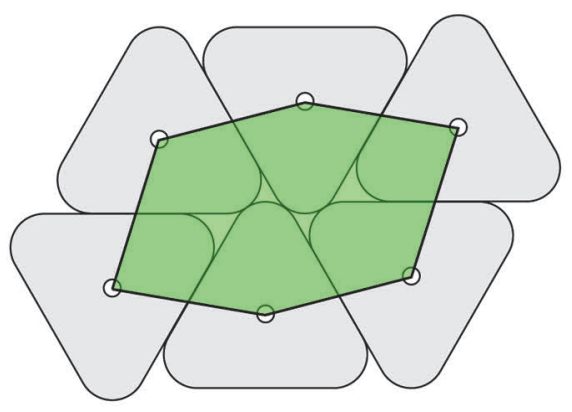

pmg (rounded)

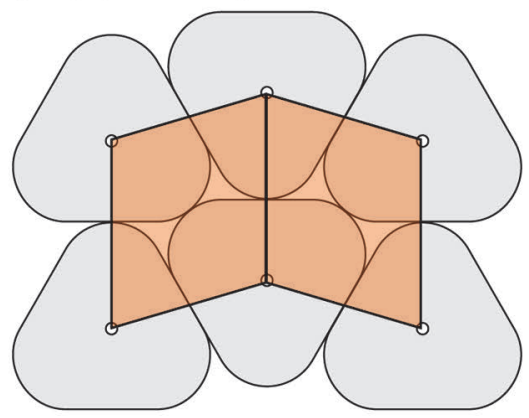

Figure S24. The coordinate polygons obtained by connecting nearest-neighbor spherotriangle centers are analyzed to automatically identify the local structure of spherotriangles found in computer simulations. The characteristic coordination polygon of the $\mathrm{p} 6 \mathrm{~m}$ phase is a regular hexagon. The characteristic coordination polygon of the p2 phase is a deformed hexagon with inversion symmetry. The characteristic coordination polygon of the pmg phase can be parallelograms (as shown) and triangles (not shown) based on the choice of nearest-neighbor distance cutoff. Because plastic crystals also form triangles, we also consider spherotriangle orientations in a second step. 

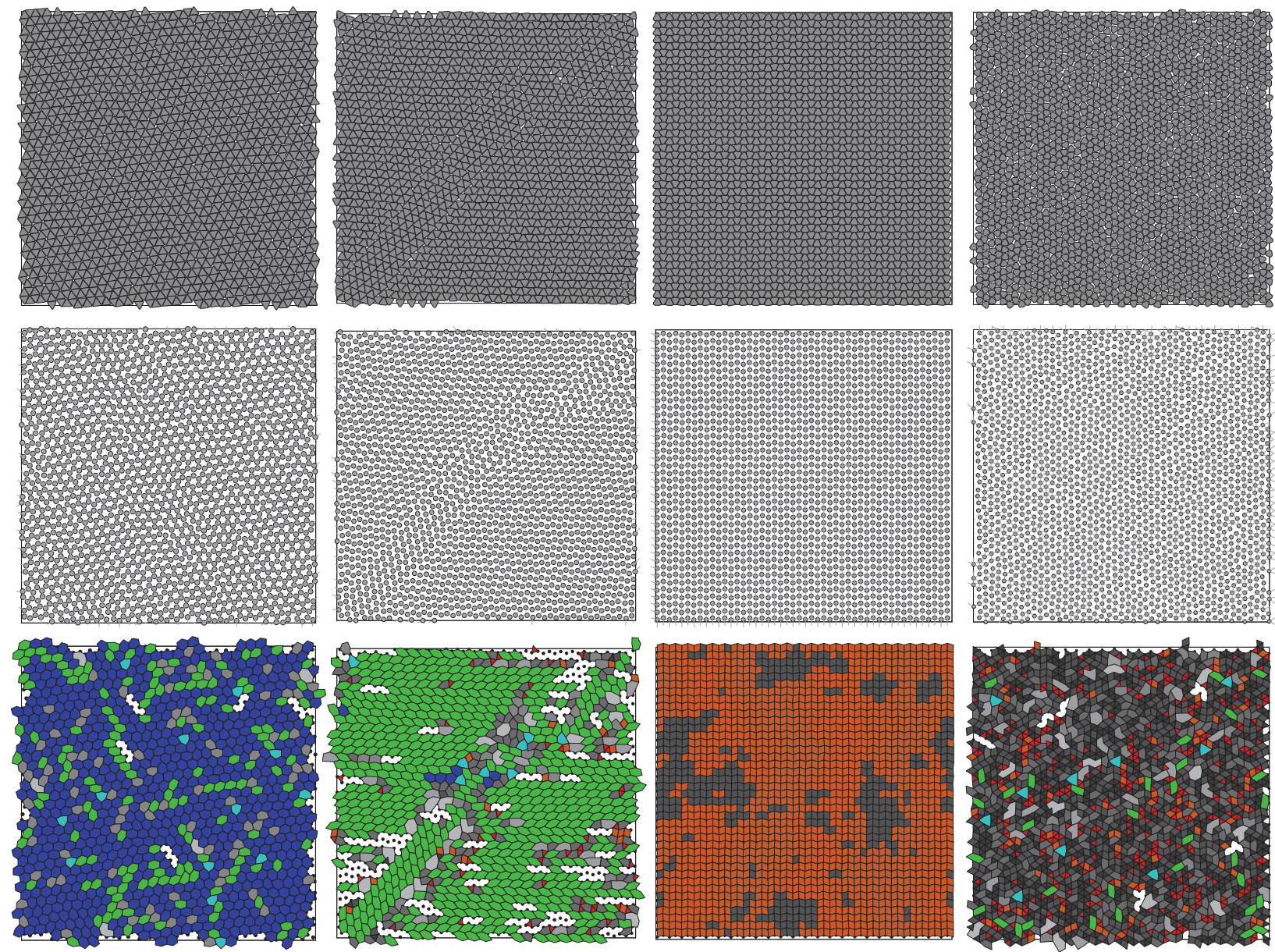

Figure S25. Examples of local environments and their analysis for the hard spherotriangle model. The first row contains one selected example from the hard spherotriangle simulations for each structure (p6m, p2, pmg, plastic). Connection of nearest-neighbor particle centers describe polygons (second row). The third row contains polygons colored by the local structure as described in Figure S24: blue for p6m, green for p2, orange, and red for pmg. All other polygons are colored by shades of gray. The same shade of gray corresponds to polygons with the same number of vertices. Polygons with eight or more vertices are not colored. Rows show simulations at the parameter sets $(R, \phi)=(0.1,0.9),(0.2,0.9),(0.3,0.9)$, and $(0.4,0.8)$. 

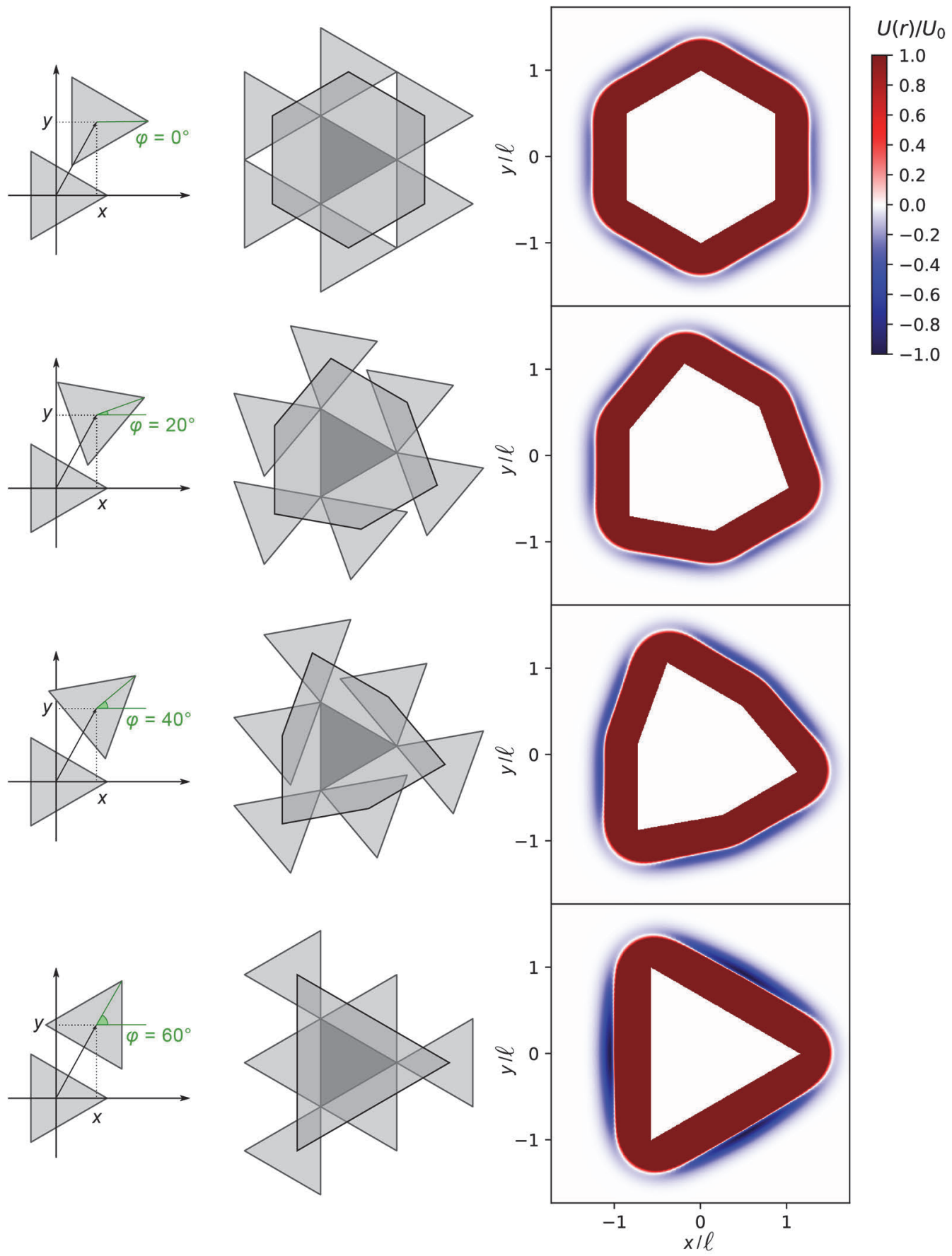

Figure S26. Pair potential maps of triangles with attractive ligand brushes described by the Derjaguin approximation are precomputed and tabulated in three-parameter tables. The parameters are relative orientation $\varphi$ and relative translation by the vector $(x, y)$ as shown in the left column, where we fix in each row the parameter $\varphi$ and vary the other two parameters $x$ and $y$. The coordinate system was chosen by fixing the first triangle and moving the second triangle, which affects the interaction area (middle column). In the potential maps (right column), red color denotes repulsive potential and blue marks attraction. Relative translations with intersecting triangles are blank. The attractive triangle model captures the rounding effect of ligand brushes and a varying interaction strength depending on the amount of ligand brush contact fully automatically. Potential parameters: rounding $R=0.2$, softness $S=0.1$. 

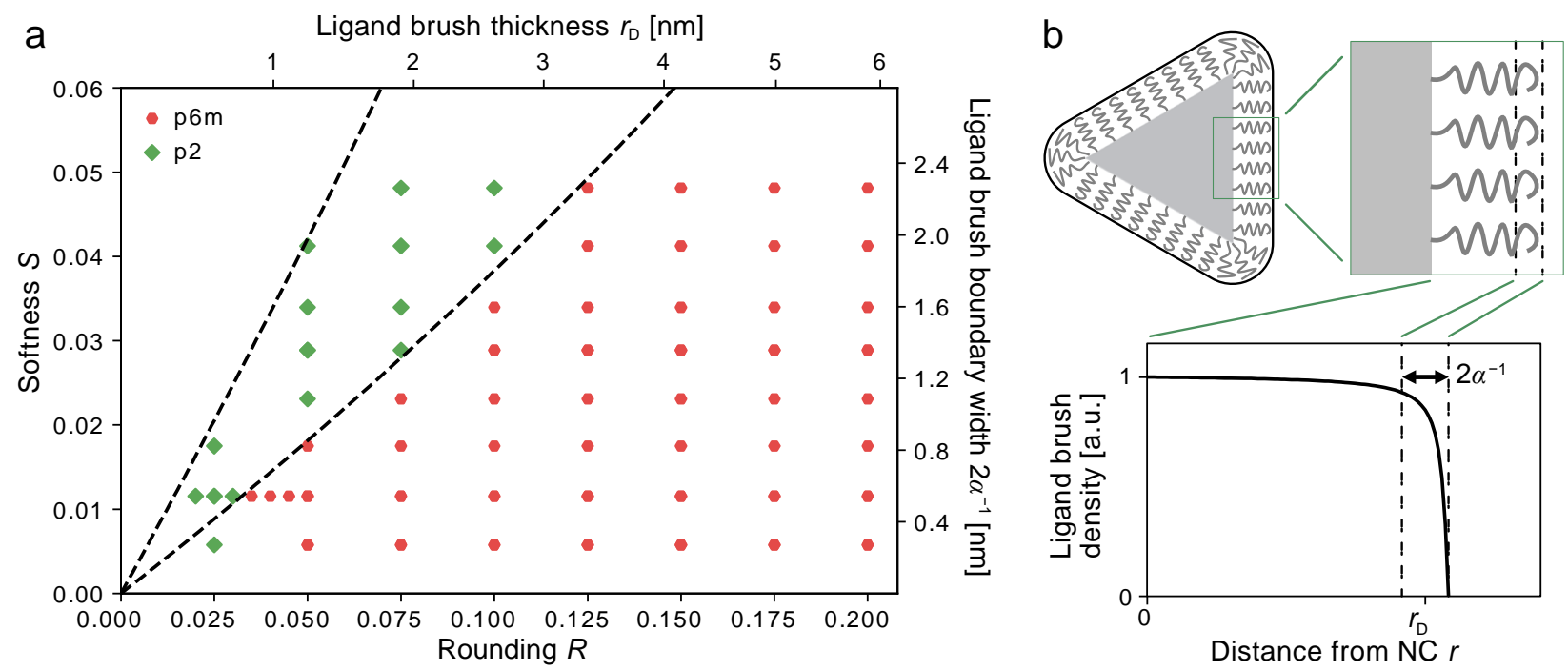

Figure S27. (a) Phase diagram of the attractive triangle model varying the ligand brush parameter softness $S=(\alpha \ell)^{-1}$ and the ligand brush shape parameter rounding $R$ at density $\phi=0.9$. Each data point originates from a simulation cooled down to $k_{\mathrm{B}} T=10^{-3} U_{0}$. The phases $\mathrm{p} 2$ and $\mathrm{p} 6 \mathrm{~m}$ are separated by a line with constant $\alpha r_{D}$. Thus, for short-range attractive forces, $\alpha^{-1} \ll \ell$, the phase diagram collapses to a line varying in $\alpha r_{D}$. We also include the derived parameter $2 \alpha^{-1}=2 S \ell$ as the right axis and the derived parameter $r_{D}=\ell R /(1-R)$ as the top axis for nanoprisms with edge length $\ell=23.5 \mathrm{~nm}$. When mapped to the experiment, these parameters can be interpreted as the thickness of the ligand brush (responsible for the rounding of the spherotriangle shape with a disk of radius $r_{\mathrm{D}}$ ) and the ligand brush boundary width (i.e., related to the steepness of the pairwise interaction potential between two attractive triangles in opposite orientation). (b) Sketch of the ligand brush around a triangular nanoprism (gray) together with the expected ligand brush density. The ligand brush density is drawn as half of a sigmoid function decaying to zero near the ligand brush thickness $r_{\mathrm{D}}$ within the distance $2 \alpha^{-1}$. 
(a)

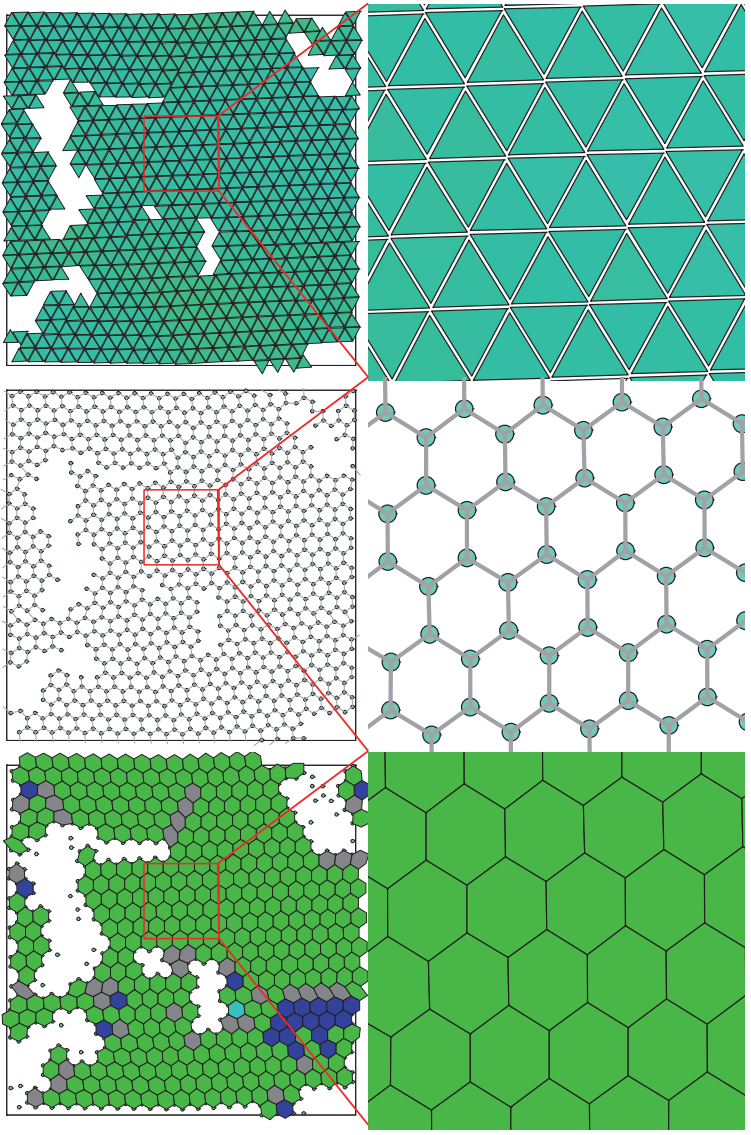

(b)

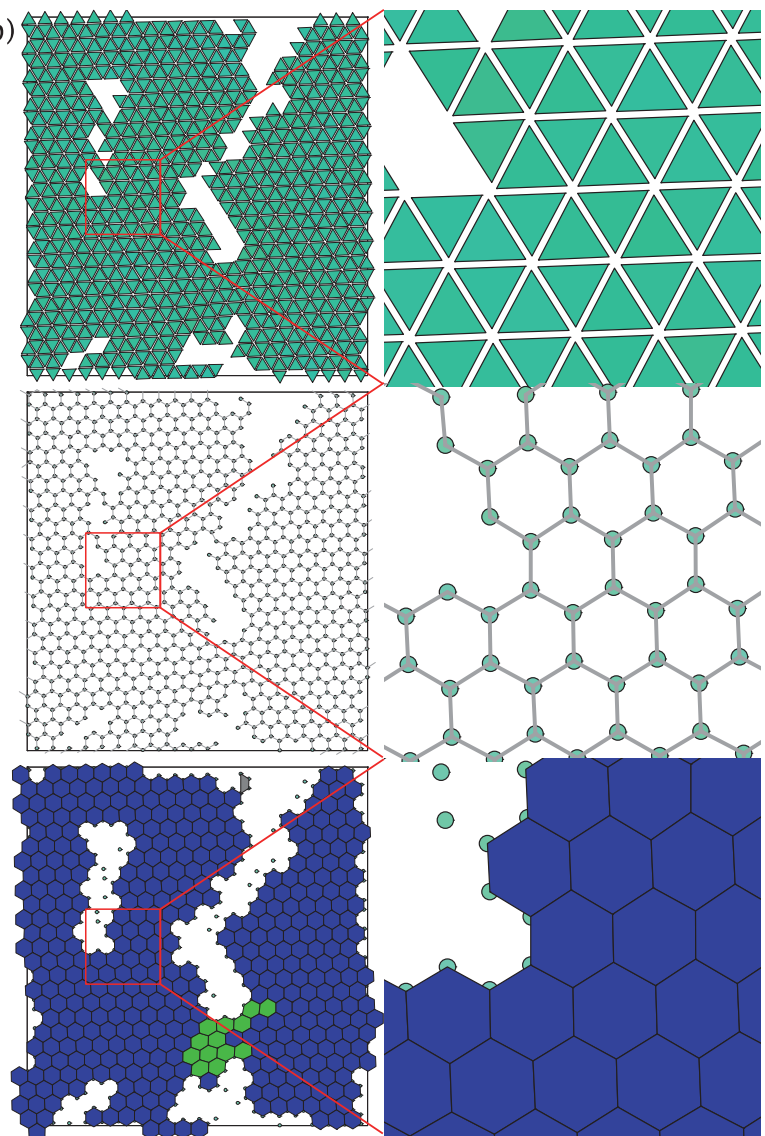

Figure S28. Examples of local environments and their analysis for the attractive triangle model with focus on the appearance of the energetically favored p2 phase. The first row contains one selected example each from the attractive triangle simulations for (a) the energetically favored p2 phase and (b) the p6m phase. The second row and the third row follow the conventions of Figure S25. The included cuts (right columns) highlight the local arrangement. The triangle vertices are packed more densely for the energetically favored p2 phase and a deviation from six-fold symmetry can be observed. This deviation is weak for the example shown here because it is the endpoint of the simulation at very low temperature (or high $U_{0}$ ), however is more pronounced in other regions of parameter space. Shown are the parameter sets $\left(R, S, U_{0} / k_{\mathrm{B}} T\right)=$ $(0.025,0.01,200)$ in (a) and $(0.050,0.01,200)$ in (b). 
(a)

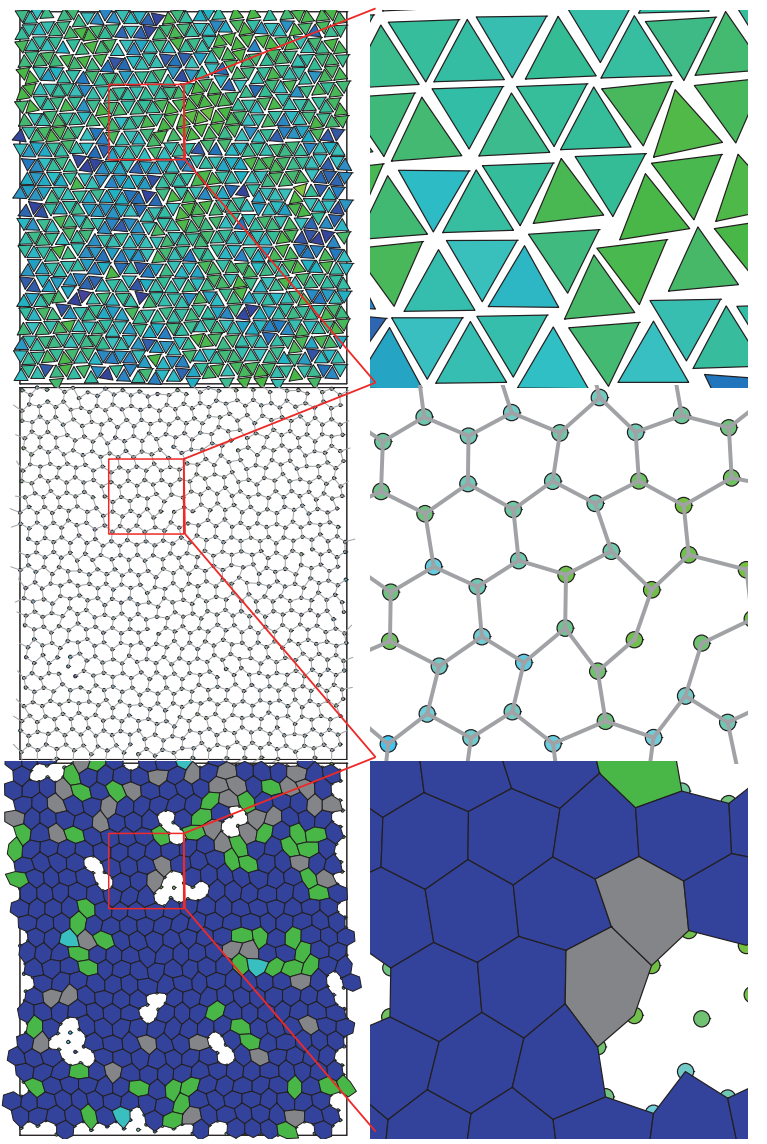

(b)

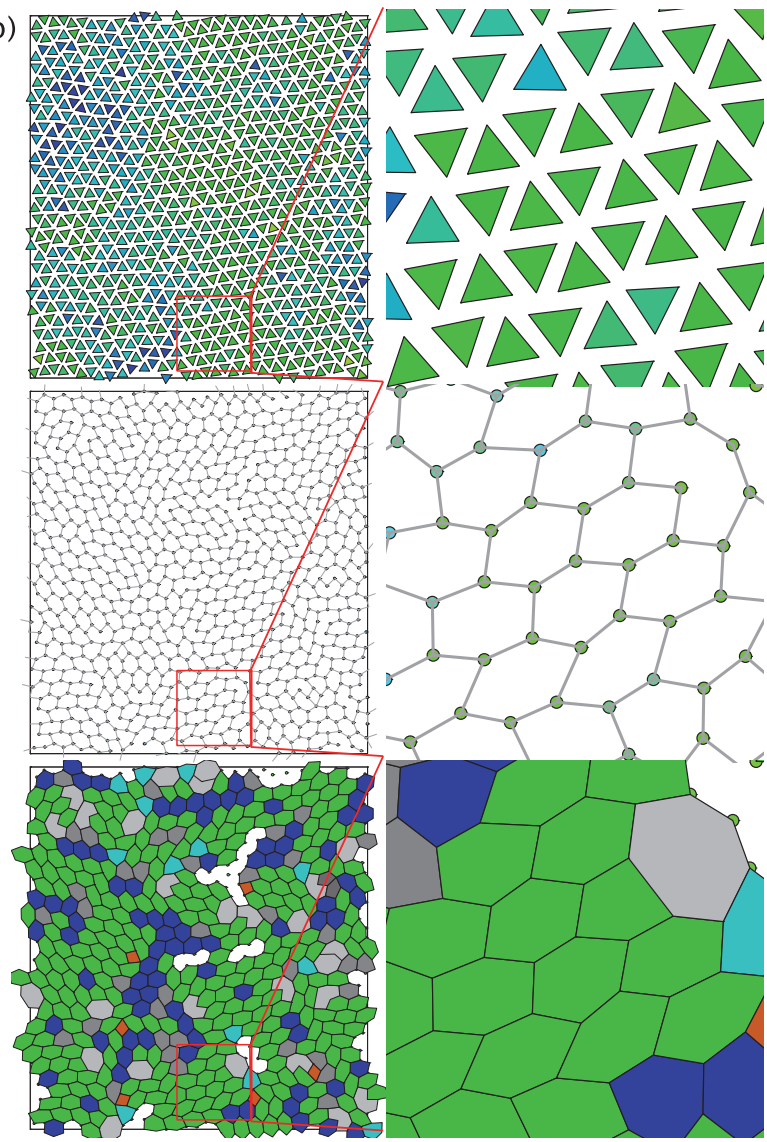

Figure S29. Examples of local environments and their analysis for the attractive triangle model with focus on the appearance of the entropically favored p2 phase. The first row contains one selected example each from the attractive triangle simulations for (a) the p6m phase and (b) the entropically favored $\mathrm{p} 2 \mathrm{phase}$. The second row and the third row follow the conventions of Figure S25. The included cuts (right columns) highlight the local arrangement. More fluctuations are possible in the p6m phase. The deviation from sixfold symmetry in the entropically favored p2 phase is larger than that of the energetically favored p2 phase shown in Figure S28. Shown are the parameter sets $\left(R, S, U_{0} / k_{\mathrm{B}} T\right)=(0.05,0.01,0.5)$ in (a) and $(0.15,0.01,0.5)$ in (b). 

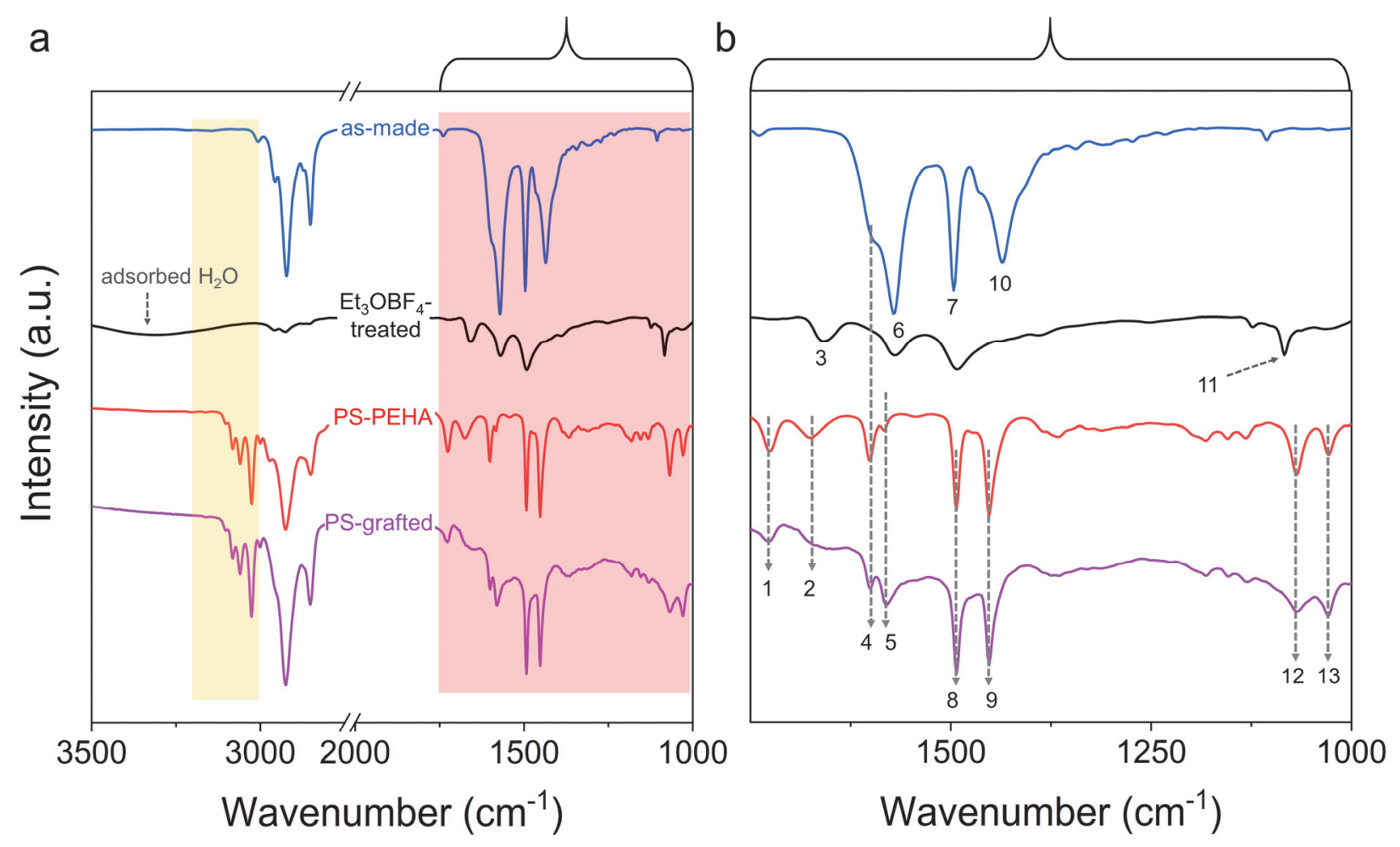

Figure S30. (a) FTIR spectra of as-made nanoprisms, $\mathrm{Et}_{3} \mathrm{OBF}_{4}$-treated nanoprisms, pure PS-PEHA ligands and PS-grafted nanoprisms obtained using the two-step ligand-exchange method. The yellow band highlights aromatic C-H stretches from PS-PEHA. (b) Expanded view of the spectral region 1750-1000 $\mathrm{cm}^{-1}$ shown in (a). 


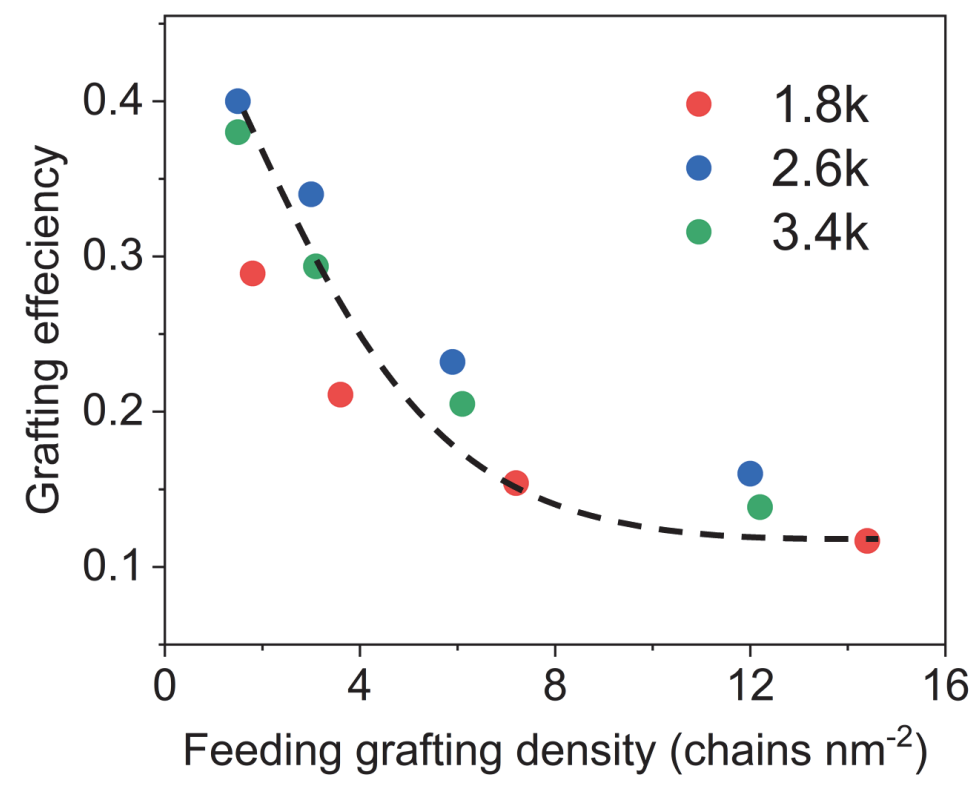

Figure S31. Plot of grafting efficiency versus feeding grafting density for three PS-PEHA ligands used for two-step ligand-exchange. The grafting efficiency of PS-PEHA is quantified as the ratio between the actual grafting density determined from TGA and the feeding grafting density. The dashed line on the graph is provided as a guide to the eyes. Raw data are shown in Table S6. The halide end-functionality of PS-Br quantified by using NMR (90\% for $1.8 \mathrm{kDa}$ PS-Br, 93\% for $2.6 \mathrm{kDa}$ PS-Br and 93\% for $3.4 \mathrm{kDa} \mathrm{PS}-\mathrm{Br}$ ) has been taken into account for calculations of grafting efficiency.
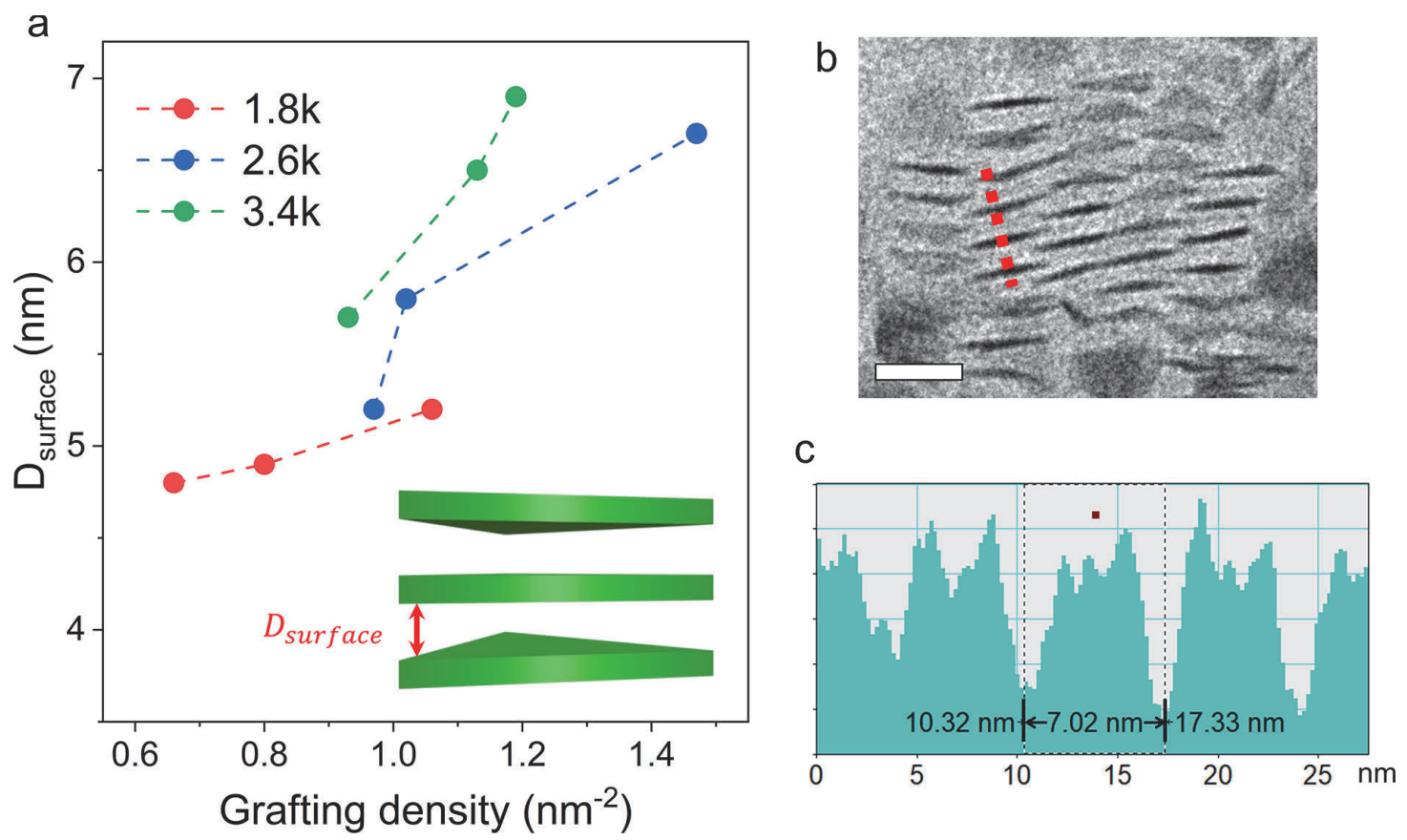

Figure S32. (a) Nearest-neighbor distances measured from vertically standing linear stacks of PS-grafted $\mathrm{Gd}_{2} \mathrm{O}_{3}$ nanoprisms (edge length: $23.5 \mathrm{~nm}$ ) prepared using the two-step ligand-exchange method. (b) An example of measurement of face-to-face distance $D_{\text {surface }}$ for $2.6 \mathrm{kDa}$ PS-grafted nanoprism stacks and (c) corresponding line profile along the red dotted line indicated in (b). Scale bar: $20 \mathrm{~nm}$. 


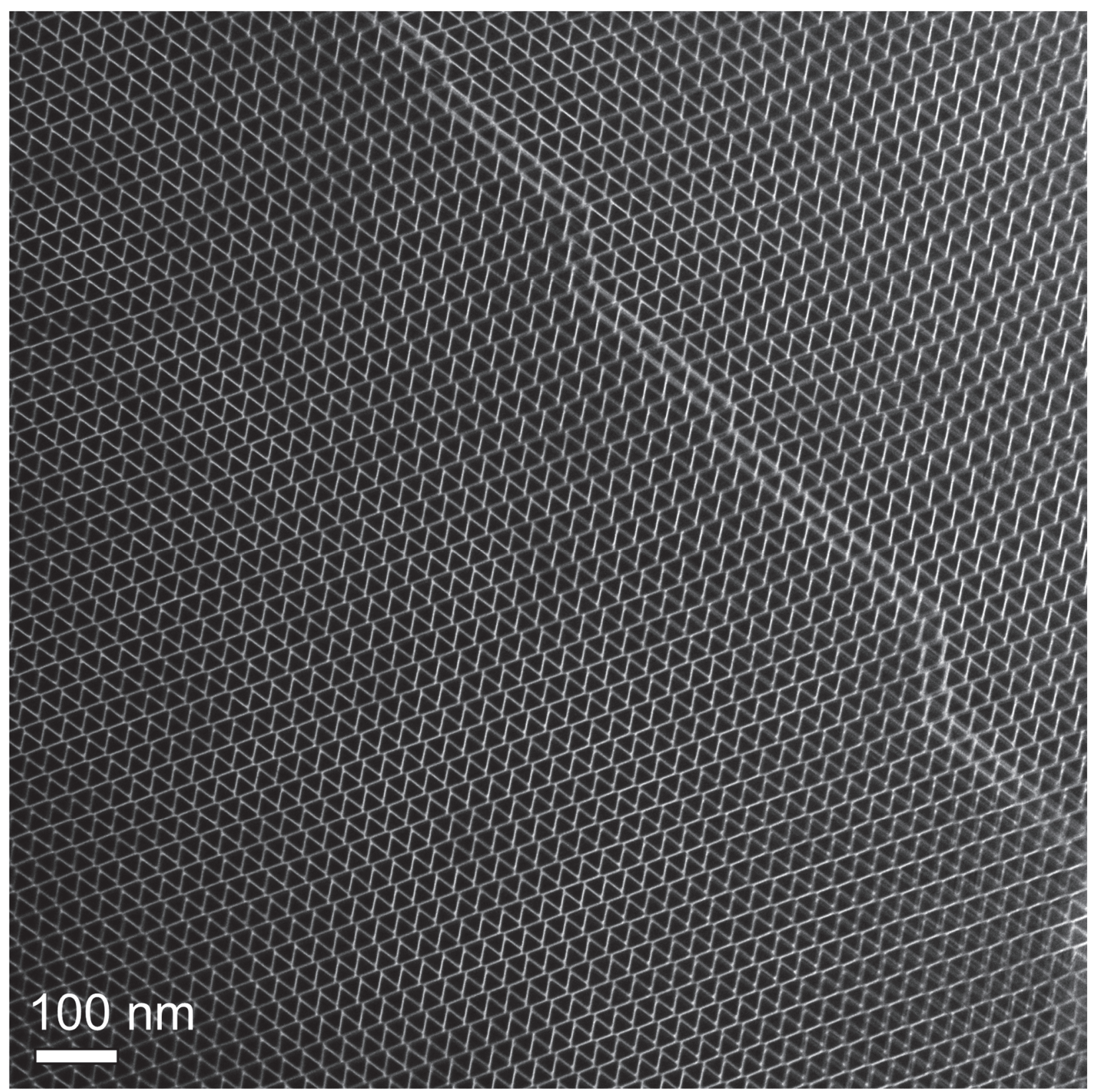

Figure S33. Low-magnification TEM image of p2-type superstructures self-assembled from $1.8 \mathrm{kDa}$ PSgrafted $\mathrm{Gd}_{2} \mathrm{O}_{3}$ nanoprisms (edge length: $23.5 \mathrm{~nm}$ ) prepared using the two-step ligand-exchange method. 


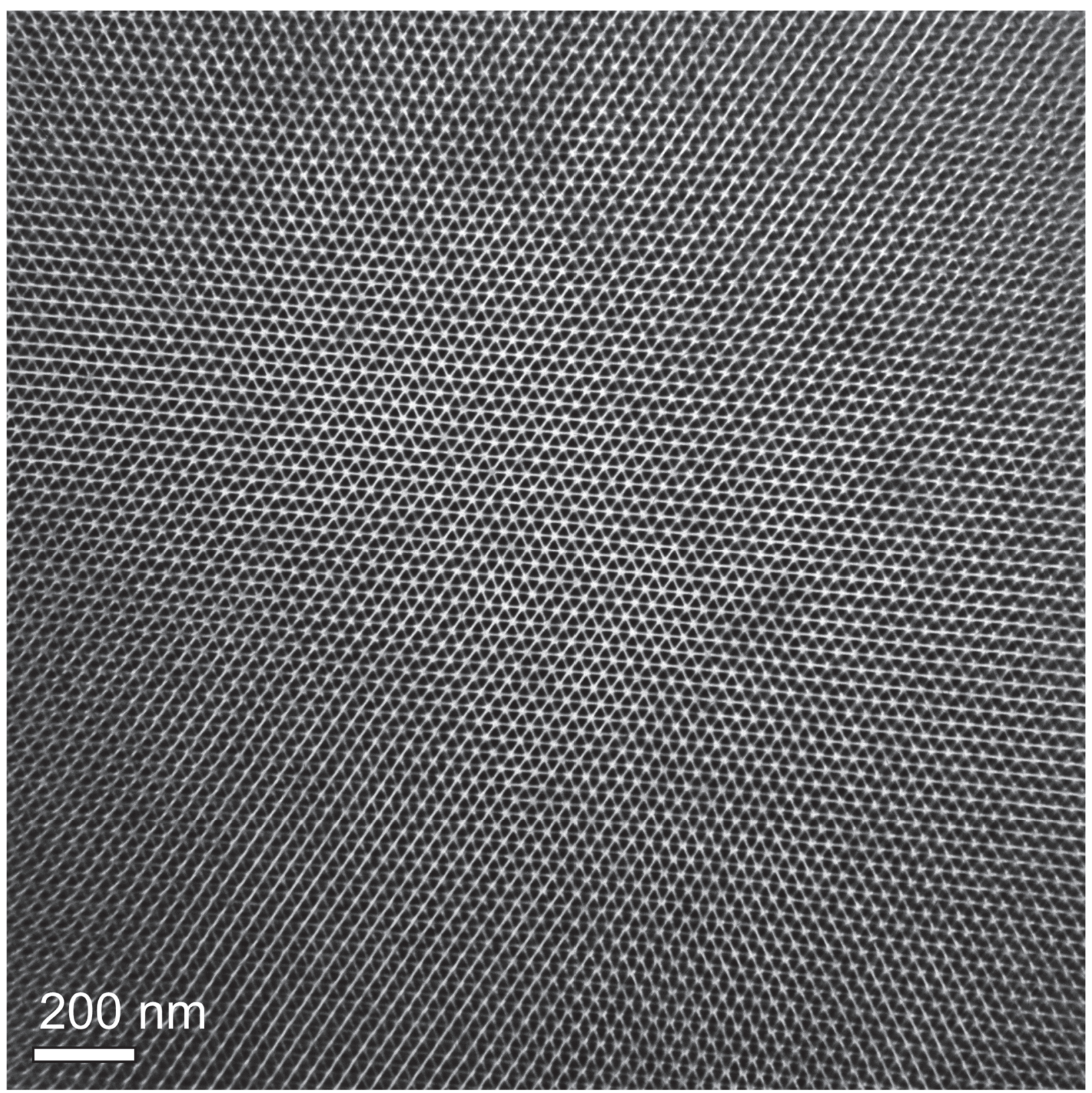

Figure S34. Low-magnification TEM image of p6m-type superstructures self-assembled from $2.6 \mathrm{kDa}$ PSgrafted $\mathrm{Gd}_{2} \mathrm{O}_{3}$ nanoprisms (edge length: $23.5 \mathrm{~nm}$ ) prepared using the two-step ligand-exchange method. 


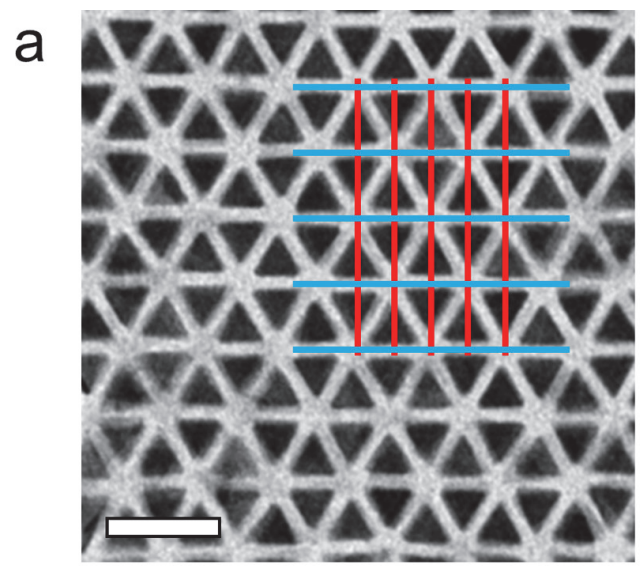

b

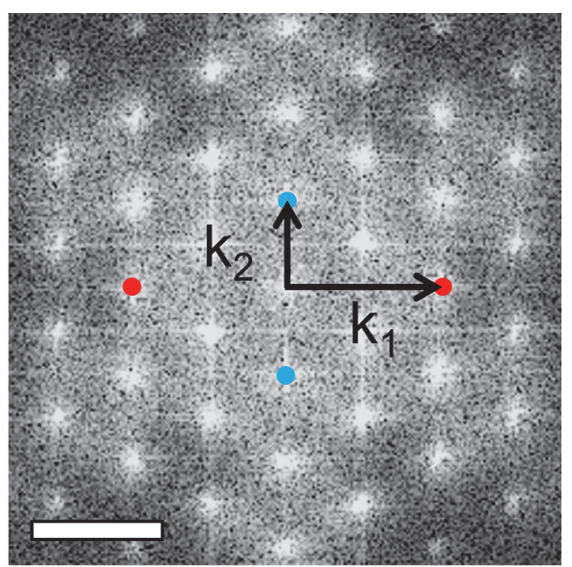

C

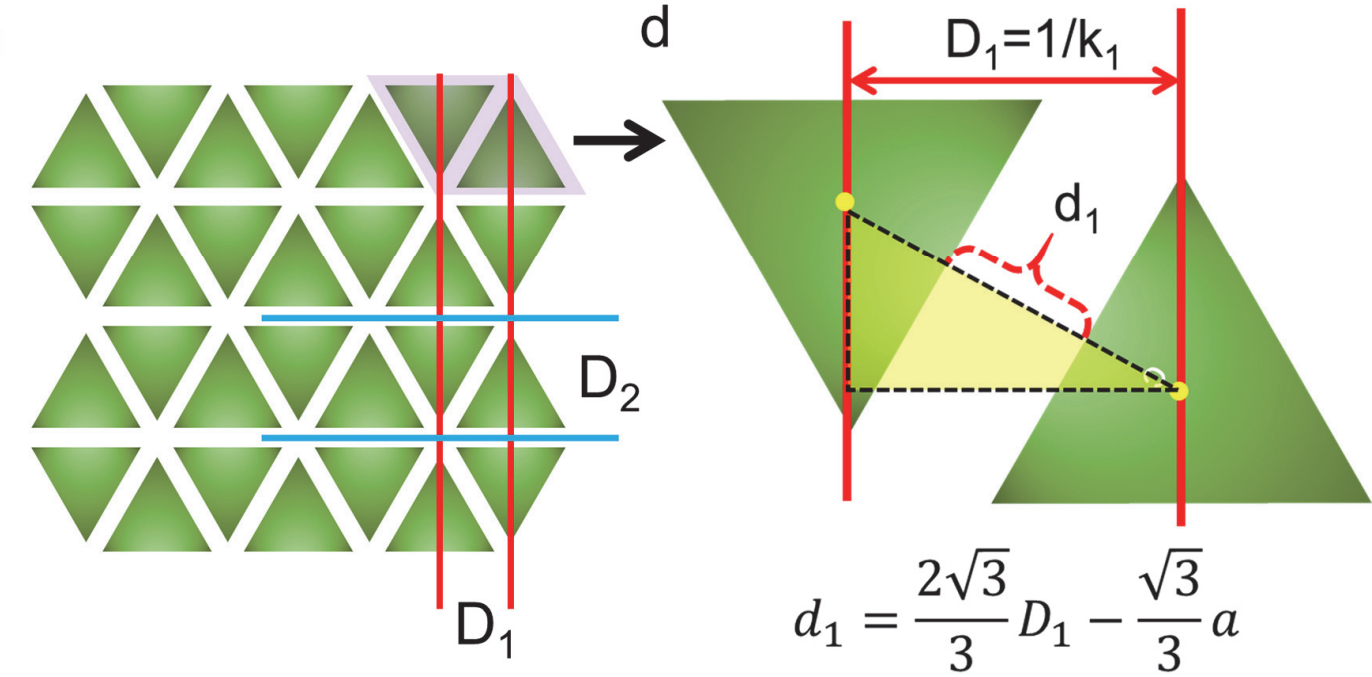

Figure S35. (a) Exemplary TEM image and (b) corresponding FFT pattern of p6m-type superstructures. The set of planes in (a) and the corresponding spots (solid circles) on the FFT pattern in (b) are color matched. (c) Definition of interplanar spacing $D_{1}$ and $D_{2}$ and (d) calculation of edge-to-edge distance between nearest-neighbor triangles $d_{1}$. Scale bars: (a) $50 \mathrm{~nm}$, (b) $0.05 \mathrm{~nm}^{-1}$. 


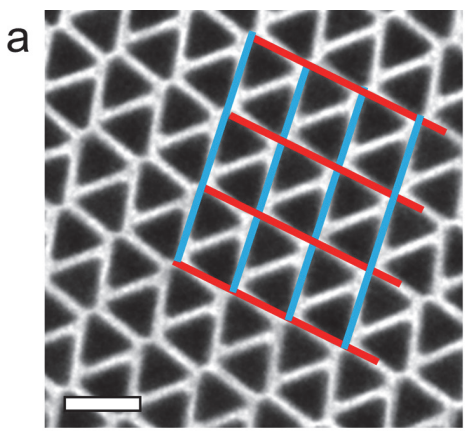

d

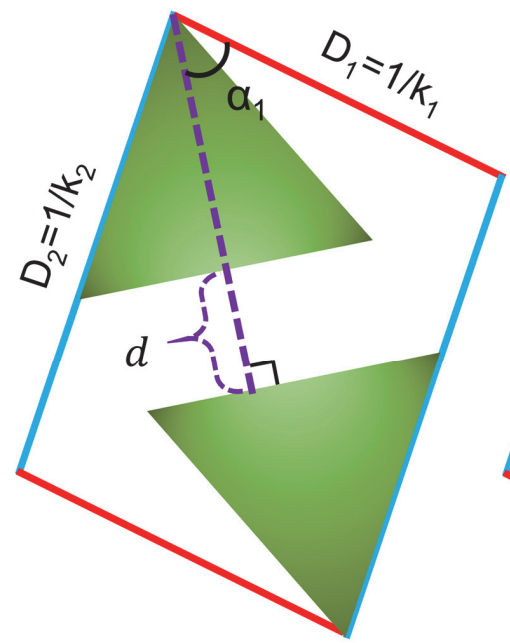

b

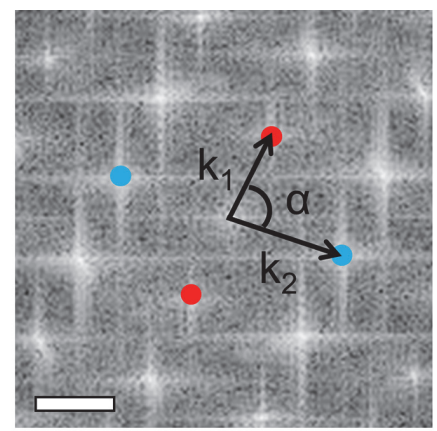

e

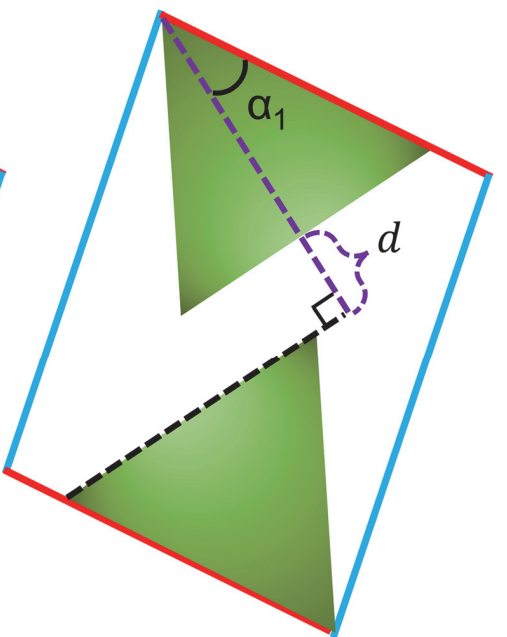

C

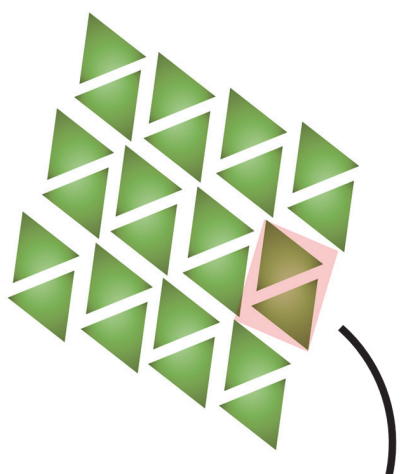

$f$

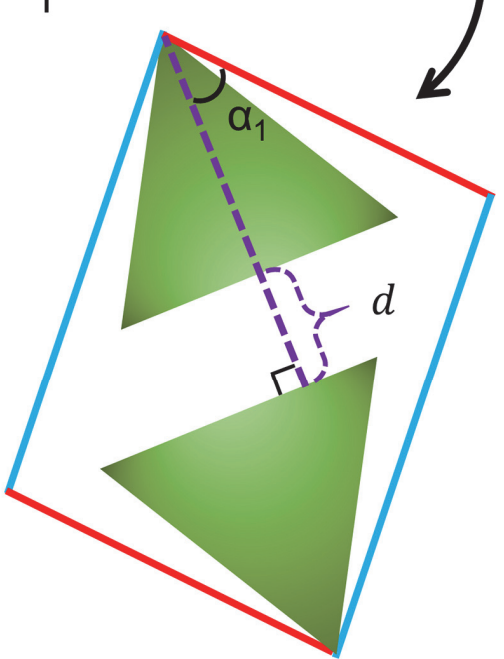

Figure S36. (a) Exemplary TEM image and (b) corresponding FFT pattern of p2-type superstructures. The set of planes in (a) and the corresponding spots (solid circles) on the FFT pattern in (b) are color matched. $\alpha$ denotes the acute angle between the red and blue lines. (c) Schematic illustration of the p2-type superstructure and an individual parallelogram (pink) from which the edge-to-edge distance between adjacent nanoprisms can be calculated. Of note, the triangles can rotate slightly within the confines of the parallelogram without altering the peak positions in the FFT pattern. (d-f) Schematic illustration of the three different orientations of the nanoprism pair with respect to the parallelogram. $\alpha_{1}$ denotes the angle between the height of the upper triangle and the red line. (d) $\alpha_{1}=\alpha_{\max }=\alpha-30^{\circ}$, (e) $\alpha_{1}=\alpha_{\min }=30^{\circ}$ and (f) $30^{\circ}<\alpha_{1}<\alpha-30^{\circ}$. The edge-to-edge distance $d$ can be calculated once properties of the parallelogram (from FFT) and the value of $\alpha_{1}$ (from TEM image) are known. Scale bars: (a) $30 \mathrm{~nm}$, (b) $0.25 \mathrm{~nm}^{-1}$. 

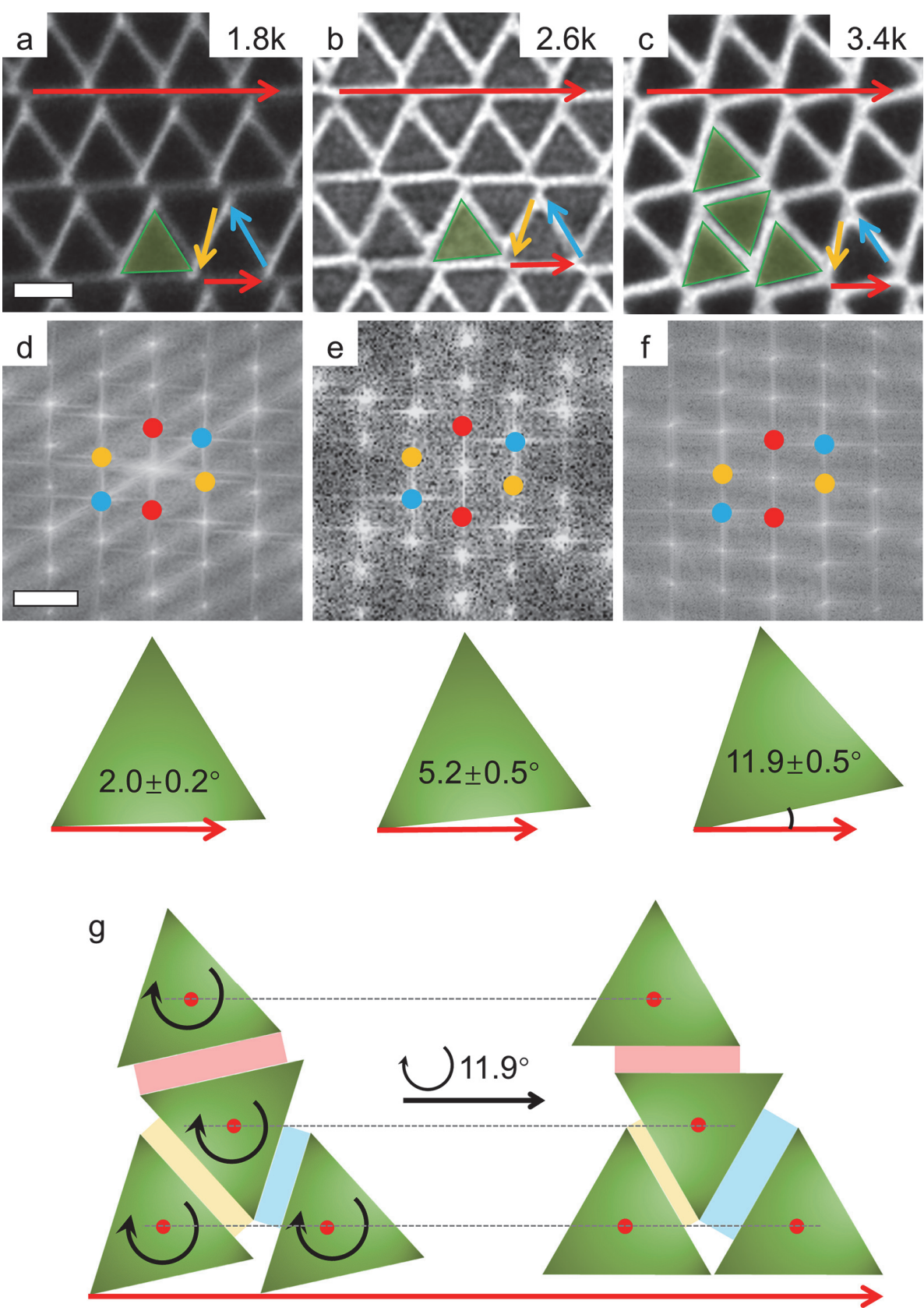

Figure S37. (a-c) Exemplary TEM images and (d-f) corresponding FFT patterns of p2-type superstructures formed with large $\mathrm{Gd}_{2} \mathrm{O}_{3}$ nanoprisms grafted with (a) $1.8 \mathrm{kDa}$, (b) $2.6 \mathrm{kDa}$ and (c) $3.4 \mathrm{kDa}$ PS-PEHA ligands. Arrows on the TEM images and the corresponding spots (solid circles) on the FFT patterns are color matched. The red dashed arrows indicate the layering direction, which is normal to the largest periodicity based on FFT analysis. In contrast to the p6m-type superstructure, an angular offset is found between the triangle edge and the layering direction for p2-type superstructures. This offset increases with PS-PEHA chain length. (g) Starting from the experimentally observed configuration in (c,f), we estimate that nearly 6\% loss in edge-to-edge overlap area will result after synchronous anti-clockwise rotation of all four triangles such that their edges become parallel to the layering direction as indicated by the red arrow. Scale bars: (a-c) $20 \mathrm{~nm}$, (d-f) $0.04 \mathrm{~nm}^{-1}$. 

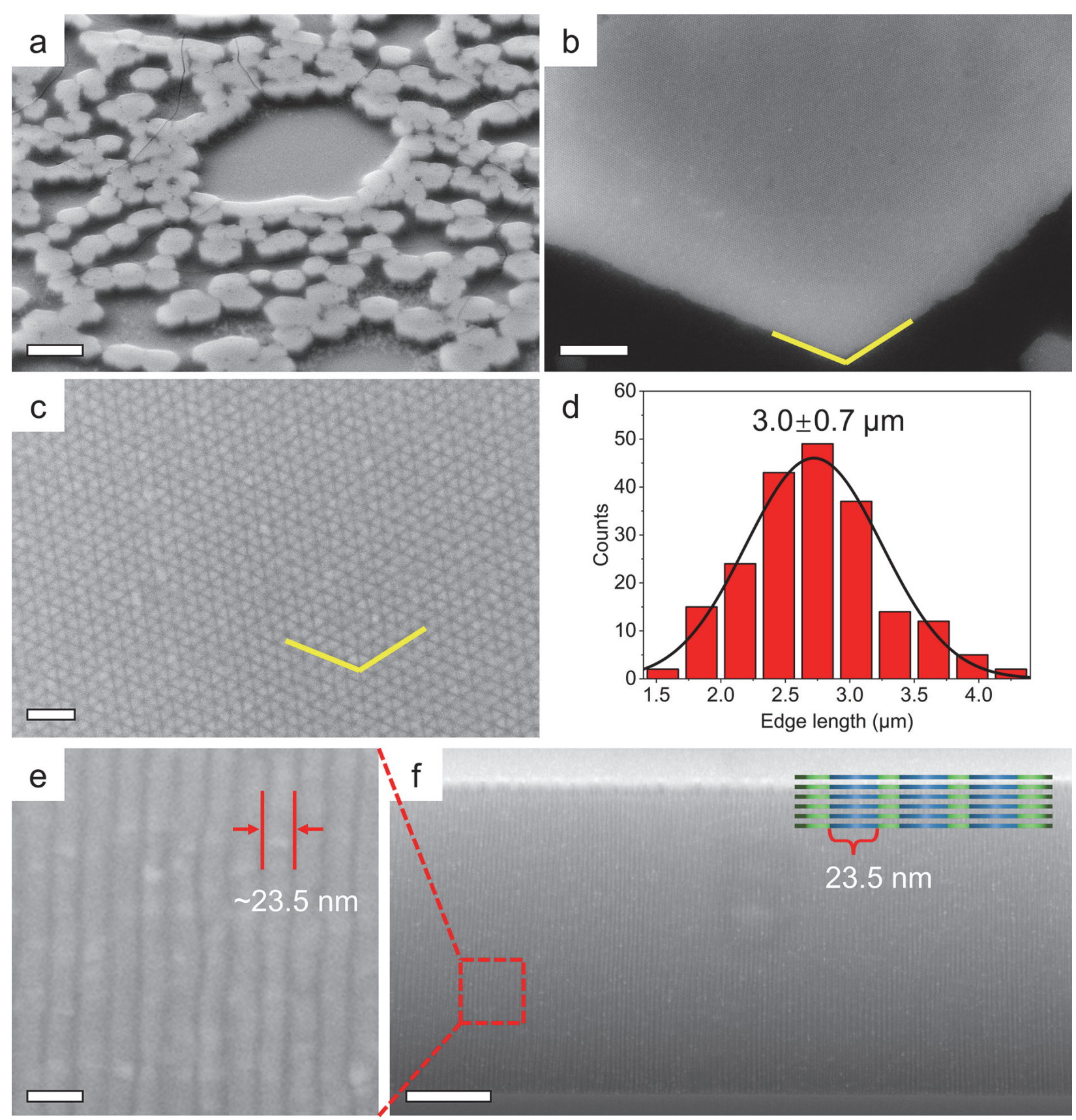

Figure S38. (a-c) Additional SEM images of hexagonal disk-shape supercrystals self-assembled from 2.6 kDa PS-grafted $\mathrm{Gd}_{2} \mathrm{O}_{3}$ nanoprisms (edge length: $23.5 \mathrm{~nm}$ ) prepared using the two-step ligand-exchange method. (d) Distribution histogram of the edge length of hexagonal disk-shape supercrystals measured from SEM images. (e, f) Cross-sectional SEM images of hexagonal disk-shape supercrystals. Inset of (f) is a side-view illustration of the $\mathrm{p} 6 \mathrm{~m}$ superstructure. The width of individual stacks (i.e., $23.5 \mathrm{~nm}$ ) measured from (e) is consistent with the edge length of individual triangular nanoprisms. Scale bars: (a) $10 \mu \mathrm{m}$, (b) 1 $\mu \mathrm{m}$, (c) $100 \mathrm{~nm}$, (e) $50 \mathrm{~nm}$, (f) $500 \mathrm{~nm}$. 


\section{direct ligand-exchange}
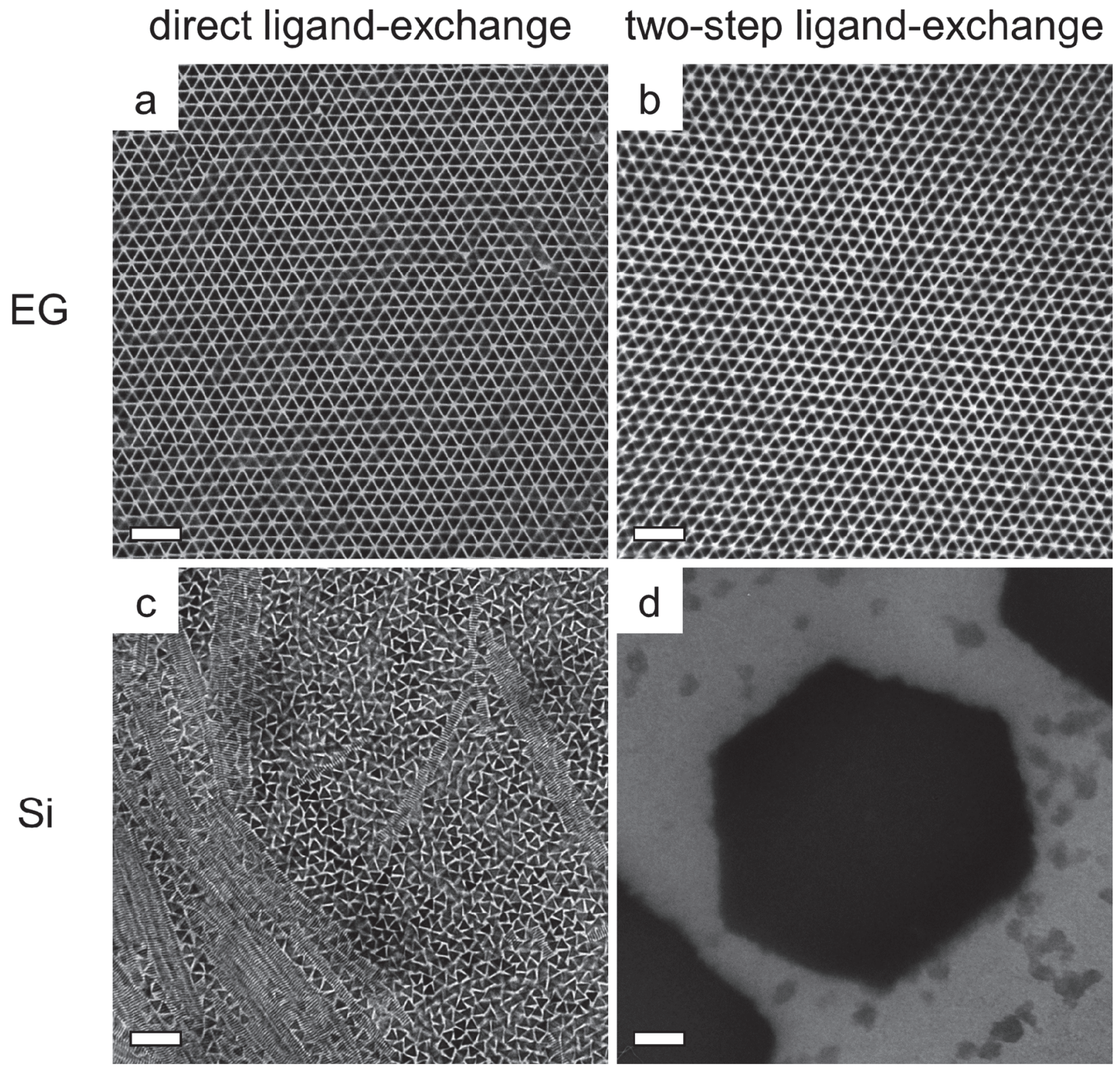

Figure S39. Comparison of TEM images of assemblies of $2.6 \mathrm{kDa}$ PS-grafted $\mathrm{Gd}_{2} \mathrm{O}_{3}$ nanoprisms (edge length: $23.5 \mathrm{~nm}$ ) prepared via the direct and the two-step ligand-exchange method. Assemblies were formed by drying a nanoprism solution on top of the EG subphase (upper row) or directly on Si substrates (bottom row). Scale bars: (a-c) $100 \mathrm{~nm}$, (d) $500 \mathrm{~nm}$. 

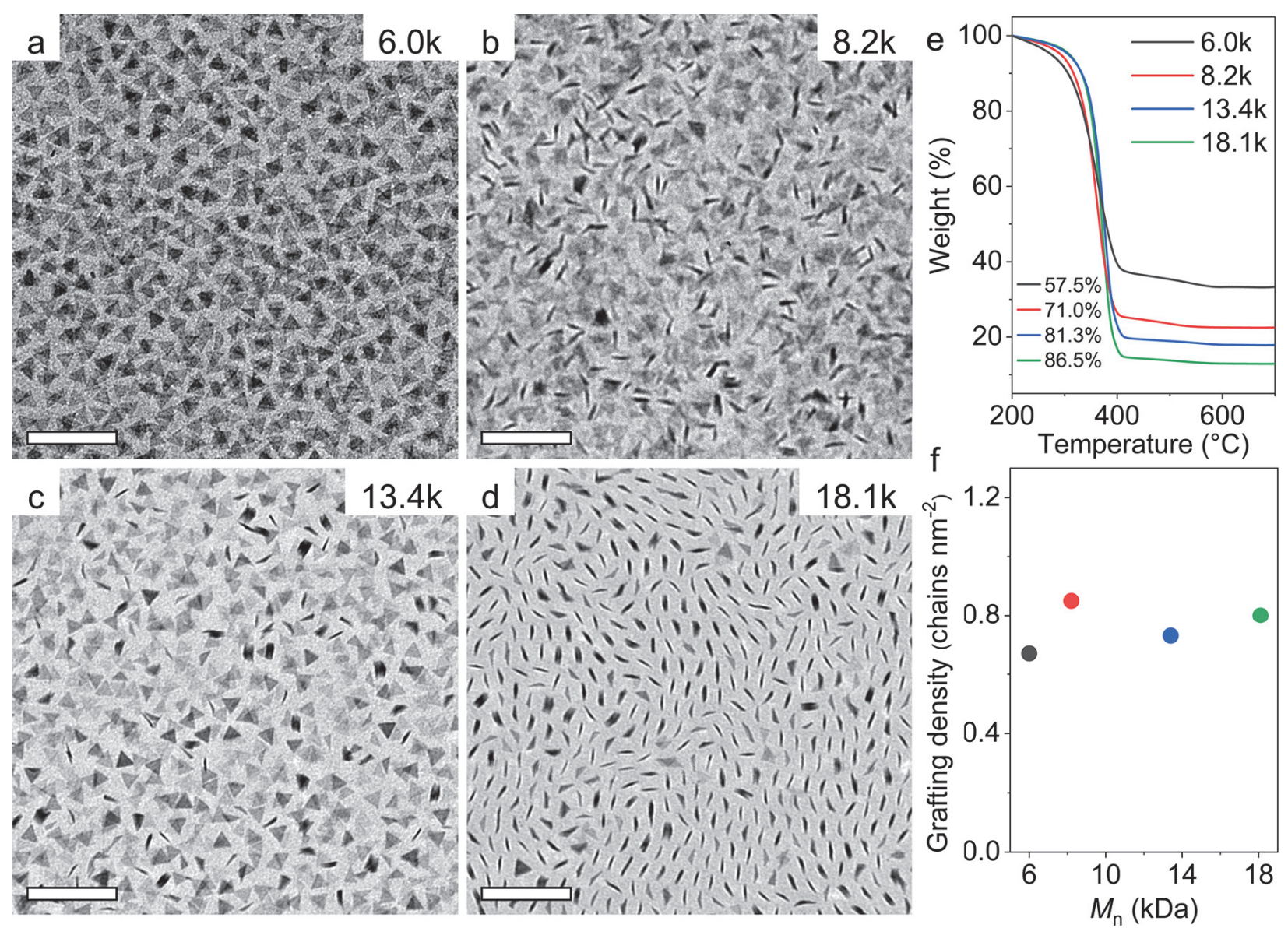

Figure S40. (a-d) Representative TEM images of assemblies of PS-grafted $\mathrm{Gd}_{2} \mathrm{O}_{3}$ nanoprisms (edge length: $23.5 \mathrm{~nm}$ ) prepared using the two-step ligand-exchange method with high-molecular-weight PS-PEHA ligands $\left(M_{n} \geq 6.0 \mathrm{kDa}\right)$. Scale bars: $100 \mathrm{~nm}$. 
Table S1. Synthetic parameters of PS-Br $\left(M_{n}>5 \mathrm{kDa}\right)$. The amount of styrene was kept at $43.6 \mathrm{mmol}$ for all syntheses. The molar ratios of EBIB : $\mathrm{CuBr}_{2}: \mathrm{Me}_{6} \mathrm{TREN}: \mathrm{Sn}(\mathrm{EH})_{2}=1: 0.01: 0.1: 0.1$. Reactions were conducted at $90{ }^{\circ} \mathrm{C}$ in the absence of any additional solvent.

\begin{tabular}{|c|c|c|c|c|c|c|c|c|c|}
\hline $\begin{array}{c}M_{n} \text { of } \\
\text { PS-PEHA } \\
(\mathrm{kDa})\end{array}$ & \multicolumn{4}{|c|}{ Molar ratio } & Time & $\begin{array}{c}\text { Monomer } \\
\text { conversion } \\
(\%)\end{array}$ & $\begin{array}{c}M_{n} \text { of } \\
\text { PS-Br } \\
(\mathrm{Da})\end{array}$ & $\begin{array}{c}M_{w} / M_{n} \text { of } \\
\mathrm{PS}-\mathrm{Br}\end{array}$ \\
\hline 6.0 & 100 & 1 & 0.01 & 0.1 & 0.1 & 17 & 56 & 5,810 & 1.12 \\
\hline 8.2 & 100 & 1 & 0.01 & 0.1 & 0.1 & 20 & 72 & 8,050 & 1.05 \\
\hline 13.4 & 200 & 1 & 0.01 & 0.1 & 0.1 & 22 & 66 & 13,263 & 1.07 \\
\hline 16.1 & 300 & 1 & 0.01 & 0.1 & 0.1 & 18 & 50 & 15,974 & 1.07 \\
\hline 18.1 & 400 & 1 & 0.01 & 0.1 & 0.1 & 17 & 44 & 17,964 & 1.06 \\
\hline
\end{tabular}

Table S2. Synthetic parameters for PS-Br $\left(M_{n}<5 \mathrm{kDa}\right)$. The amount of styrene was kept at $43.6 \mathrm{mmol}$ for all syntheses. The molar ratios of EBIB : $\mathrm{CuBr}_{2}: \mathrm{Me}_{6} \mathrm{TREN}: \mathrm{Sn}(\mathrm{EH})_{2}=1: 0.01: 0.1: 0.1$. Reactions were conducted at $90{ }^{\circ} \mathrm{C}$ in toluene.

\begin{tabular}{|c|c|c|c|c|c|c|c|c|c|}
\hline \multirow{2}{*}{$\begin{array}{c}M_{n} \text { of } \\
\text { PS-PEHA } \\
(\mathrm{kDa})\end{array}$} & \multicolumn{5}{|c|}{ Molar ratio } & \multirow{2}{*}{$\begin{array}{l}\text { Time } \\
\text { (h) }\end{array}$} & \multirow{2}{*}{$\begin{array}{c}\text { Monomer } \\
\text { conversion } \\
(\%)\end{array}$} & \multirow{2}{*}{$\begin{array}{c}M_{n} \text { of PS-Bi } \\
\text { (Da) }\end{array}$} & \multirow{2}{*}{$\begin{array}{c}M_{w} / M_{n} \text { of } \\
\text { PS-Br }\end{array}$} \\
\hline & Styrene & EBIB & $\mathrm{CuBr}_{2}$ & Me 6 TREN & $\mathrm{Sn}(\mathrm{EH})_{2}$ & & & & \\
\hline 1.8 & 20 & 1 & 0.01 & 0.1 & 0.1 & 14 & 71 & 1,644 & 1.09 \\
\hline 2.6 & 30 & 1 & 0.01 & 0.1 & 0.1 & 20 & 73 & 2,485 & 1.15 \\
\hline 3.4 & 50 & 1 & 0.01 & 0.1 & 0.1 & 17 & 59 & 3,241 & 1.15 \\
\hline
\end{tabular}

Note: The volume ratios of styrene to toluene during syntheses were 1:1, 1:1, and 2:1 for $1.8 \mathrm{kDa}, 2.6 \mathrm{kDa}$ and $3.4 \mathrm{kDa}$ PS, respectively. 
Table S3. Summary of experimental parameters used for direct ligand-exchange between as-made $\mathrm{Gd}_{2} \mathrm{O}_{3}$ nanoprisms and PS-PEHA ligands.

\begin{tabular}{|c|c|c|c|c|c|}
\hline & \multicolumn{2}{|c|}{$\mathrm{Gd}_{2} \mathrm{O}_{3}$ nanoprisms } & \multicolumn{2}{|c|}{ PS-PEHA } & \multirow{2}{*}{$\begin{array}{l}\text { feeding grafting density } \\
\text { (chains per } \mathrm{nm}^{2} \text { ) }\end{array}$} \\
\hline & $\begin{array}{l}\text { edge length } \\
\text { (nm) }\end{array}$ & mass (mg) & $M_{n}(\mathrm{kDa})$ & mass (mg) & \\
\hline Figure 2c & 23.5 & 2.0 & 1.8 & 16.0 & 16.9 \\
\hline Figure 2d & 23.5 & 2.0 & 2.6 & 24.0 & 17.3 \\
\hline Figure 2e & 23.5 & 2.0 & 6.0 & 24.0 & 7.6 \\
\hline Figure $2 f$ & 23.5 & 2.0 & 8.2 & 21.5 & 5.0 \\
\hline Figure $2 \mathrm{~g}$ & 17.0 & 2.0 & 8.2 & 21.5 & 4.7 \\
\hline Figure $2 \mathrm{~h}$ & 17.0 & 2.0 & 13.4 & 34.0 & 4.5 \\
\hline
\end{tabular}


Table S4. Summary of binding energies per adsorbates on the (001) and (100) surfaces of hexagonal phase $\mathrm{Gd}_{2} \mathrm{O}_{3}$ obtained from DFT calculations.

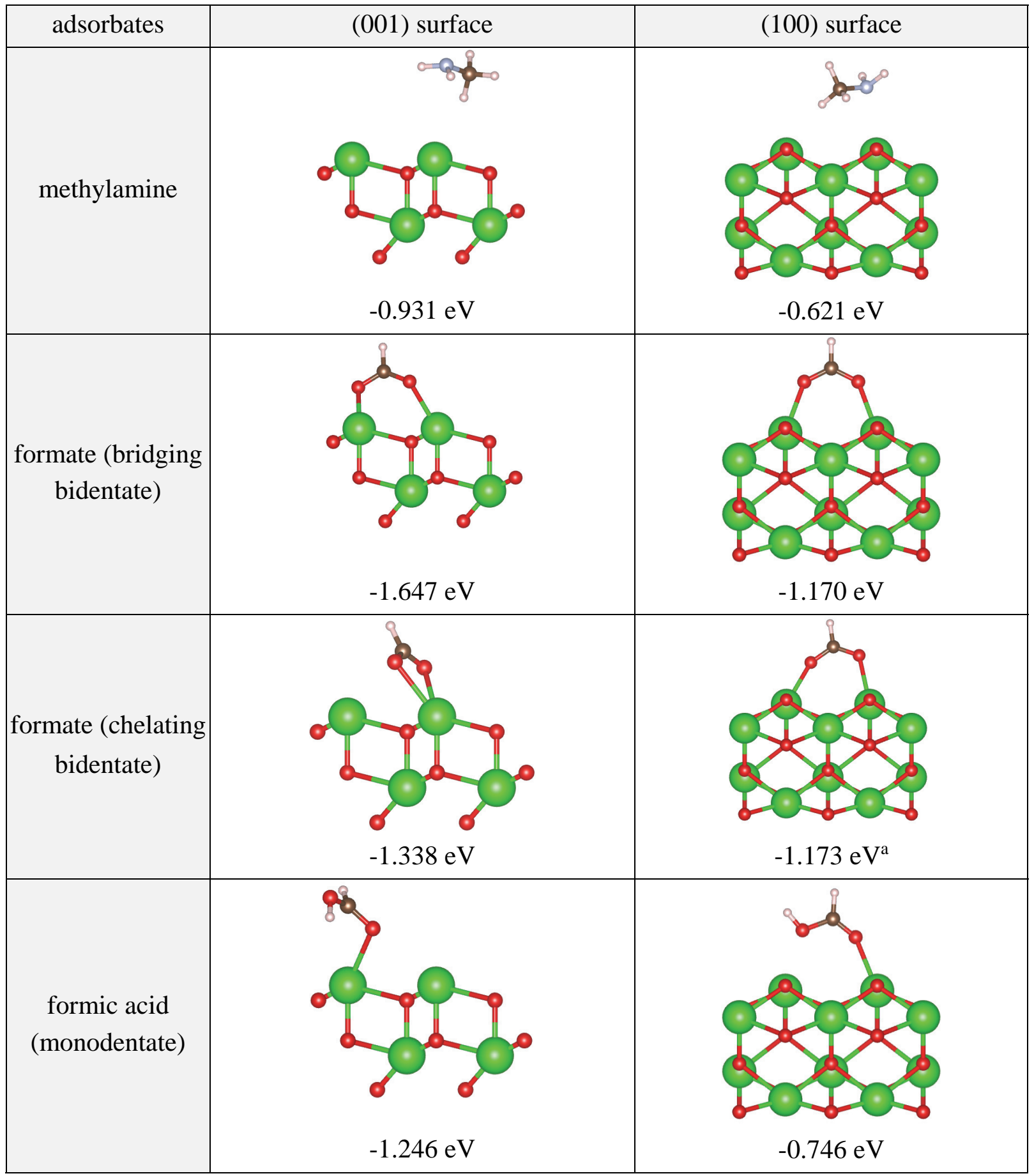

a Our DFT calculations indicate that the chelating bidentate binding mode is unstable for formate adsorbate on the $\mathrm{Gd}_{2} \mathrm{O}_{3}(100)$ surface. Instead, formate adopts a bridging bidentate binding mode upon relaxation even if the initial binding motif was set to be chelating bidentate. 
Table S5. Summary of experimental parameters used for direct ligand-exchange between OLAM-capped Au nanocrystals and PS-PEHA ligands.

\begin{tabular}{|c|c|c|c|c|c|c|}
\hline & \multicolumn{2}{|c|}{ Au nanocrystals } & \multicolumn{2}{|c|}{ PS-PEHA } & \multirow{2}{*}{$\begin{array}{l}\text { feeding grafting } \\
\text { density } \\
\text { (chains per } \mathrm{nm}^{2} \text { ) }\end{array}$} & \multirow{2}{*}{$\begin{array}{l}\text { NC center-to- } \\
\text { center distance } \\
\text { from TEM }(\mathrm{nm})\end{array}$} \\
\hline & $\begin{array}{c}\text { diameter } \\
\text { (nm) }\end{array}$ & $\begin{array}{l}\text { mass } \\
(\mathrm{mg})\end{array}$ & $M_{n}(\mathrm{kDa})$ & mass (mg) & & \\
\hline Figure S17a & 4.5 & 1.0 & 1.8 & 2.9 & 20.1 & 8.2 \\
\hline Figure S17b & 4.5 & 1.0 & 2.6 & 4.2 & 19.8 & 9.1 \\
\hline Figure S17c & 4.5 & 1.0 & 6.0 & 9.6 & 20.0 & 9.8 \\
\hline Figure S17d & 4.5 & 1.0 & 8.2 & 13.1 & 19.9 & 10.8 \\
\hline Figure S17e & 4.5 & 1.0 & 13.4 & 21.4 & 19.9 & 13.2 \\
\hline Figure S17f & 4.5 & 1.0 & 16.1 & 25.8 & 19.9 & 14.9 \\
\hline Figure S17g & 4.5 & 1.0 & 18.1 & 29.0 & 19.9 & 15.5 \\
\hline
\end{tabular}

Table S6. Summary of experimental parameters used for two-step ligand-exchange between as-made $\mathrm{Gd}_{2} \mathrm{O}_{3}$ nanoprisms (edge length: $23.5 \mathrm{~nm}$ ) and PS-PEHA ligands.

\begin{tabular}{|c|c|c|c|c|}
\hline & $\begin{array}{c}M_{n} \text { of } \\
\text { PS-PEHA } \\
(\mathrm{kDa})\end{array}$ & $\begin{array}{l}\text { Mass of } \\
\text { PS-PEHA } \\
\text { (mg) }\end{array}$ & $\begin{array}{l}\text { Feeding grafting } \\
\text { density } \\
\text { (chains per } \mathrm{nm}^{2} \text { ) }\end{array}$ & $\begin{array}{c}\text { Grafting density } \\
\text { estimated from TGA } \\
\text { (chains per } \mathrm{nm}^{2} \text { ) }\end{array}$ \\
\hline Figure $4 \mathrm{e}$ & \multirow{4}{*}{1.8} & 2.5 & 1.8 & 0.52 \\
\hline Figure 4d & & 5.0 & 3.6 & 0.76 \\
\hline Figure 4c & & 10.0 & 7.2 & 1.11 \\
\hline Figure $4 b$ & & 20.0 & 14.4 & 1.68 \\
\hline Figure $4 \mathrm{i}$ & \multirow{4}{*}{2.6} & 3.0 & 1.5 & 0.60 \\
\hline Figure 4h & & 6.0 & 3.0 & 1.02 \\
\hline Figure 4g & & 12.0 & 5.9 & 1.37 \\
\hline Figure 4f & & 24.0 & 12.0 & 1.92 \\
\hline Figure $4 \mathrm{~m}$ & \multirow{4}{*}{3.4} & 4.0 & 1.5 & 0.57 \\
\hline Figure 4l & & 8.0 & 3.1 & 0.91 \\
\hline Figure $4 \mathrm{k}$ & & 16.0 & 6.1 & 1.25 \\
\hline Figure 4j & & 32.0 & 12.2 & 1.69 \\
\hline
\end{tabular}

Note: The mass of $\mathrm{Gd}_{2} \mathrm{O}_{3}$ nanoprisms was kept at $2 \mathrm{mg}$ for all experiments described in this Table. 


\section{References}

(1) Jakubowski, W.; Kirci-Denizli, B.; Gil, R. R. and Matyjaszewski, K. Polystyrene with improved chain-end functionality and higher molecular weight by ARGET ATRP. Macromol. Chem. Phys. 2008, 209, 32-39.

(2) Wang, Y.; Chen, J.; Zhu, C.; Zhu, B.; Jeong, S.; Yi, Y.; Liu, Y.; Fiadorwu, J.; He, P. and Ye, $X$. Kinetically controlled self-assembly of binary polymer-grafted nanocrystals into ordered superstructures via solvent vapor annealing. Nano Lett. 2021, 21, 5053-5059.

(3) Paik, T.; Gordon, T. R.; Prantner, A. M.; Yun, H. and Murray, C. B. Designing tripodal and triangular gadolinium oxide nanoplates and self-assembled nanofibrils as potential multimodal bioimaging probes. ACS Nano 2013, 7, 2850-2859.

(4) Ahmad, M. W.; Xu, W.; Kim, S. J.; Baeck, J. S.; Chang, Y.; Bae, J. E.; Chae, K. S.; Park, J. A.; Kim, T. J. and Lee, G. H. Potential dual imaging nanoparticle: $\mathrm{Gd}_{2} \mathrm{O}_{3}$ nanoparticle. Sci. Rep. 2015, 5, 8549.

(5) Kim, C. R.; Baeck, J. S.; Chang, Y.; Bae, J. E.; Chae, K. S. and Lee, G. H. Ligand-size dependent water proton relaxivities in ultrasmall gadolinium oxide nanoparticles and in vivo T1 MR images in a 1.5 T MR field. Phys. Chem. Chem. Phys. 2014, 16, 19866-19873.

(6) Cai, W.; Chen, Q.; Cherepy, N.; Dooraghi, A.; Kishpaugh, D.; Chatziioannou, A.; Payne, S.; Xiang, W. and Pei, Q. Synthesis of bulk-size transparent gadolinium oxide-polymer nanocomposites for gamma ray spectroscopy. J. Mater. Chem. C. 2013, 1, 1970-1976.

(7) Ye, X.; Zhu, C.; Ercius, P.; Raja, S. N.; He, B.; Jones, M. R.; Hauwiller, M. R.; Liu, Y.; Xu, T. and Alivisatos, A. P. Structural diversity in binary superlattices self-assembled from polymergrafted nanocrystals. Nat. Commun. 2015, 6, 10052.

(8) Matthiesen, J. E.; Jose, D.; Sorensen, C. M. and Klabunde, K. J. Loss of hydrogen upon exposure of thiol to gold clusters at low temperature. J. Am. Chem. Soc. 2012, 134, 9376-9379.

(9) Wang, Y.; Neyman, A.; Arkhangelsky, E.; Gitis, V.; Meshi, L. and Weinstock, I. A. Selfassembly and structure of directly imaged inorganic-anion monolayers on a gold nanoparticle. $J$. Am. Chem. Soc. 2009, 131, 17412-17422.

(10)Nelson, A.; Zong, Y. X.; Fritz, K. E.; Suntivich, J. and Robinson, R. D. Assessment of soft ligand removal strategies: alkylation as a promising alternative to high-temperature treatments for colloidal nanoparticle surfaces. ACS Mater. Lett. 2019, 1, 177-184.

(11) Mourdikoudis, S. and Liz-Marzan, L. M. Oleylamine in nanoparticle synthesis. Chem. Mater. 2013, 25, 1465-1476.

(12)Olmos, D.; Martin, E. V. and Gonzalez-Benito, J. New molecular-scale information on polystyrene dynamics in PS and PS-BaTiO 3 composites from FTIR spectroscopy. Phys. Chem. Chem. Phys. 2014, 16, 24339-24349.

(13) Xu, Z. X. and Hu, G. X. Simple and green synthesis of monodisperse silver nanoparticles and surface-enhanced Raman scattering activity. RSC Adv. 2012, 2, 11404-11409. 
(14)Perez De Berti, I. O.; Cagnoli, M. V.; Pecchi, G.; Alessandrini, J. L.; Stewart, S. J.; Bengoa, J. F. and Marchetti, S. G. Alternative low-cost approach to the synthesis of magnetic iron oxide nanoparticles by thermal decomposition of organic precursors. Nanotechnology 2013, 24, 175601.

(15)Seyhan, M.; Kucharczyk, W.; Yarar, U. E.; Rickard, K.; Rende, D.; Baysal, N.; Bucak, S. and Ozisik, R. Interfacial surfactant competition and its impact on poly(ethylene oxide)/Au and poly(ethylene oxide)/Ag nanocomposite properties. Nanotechnol. Sci. Appl. 2017, 10, 69-77.

(16) Socrates, G. Infrared and Raman Characteristic Group Frequencies: Tables and Charts. Ch. 9, (John Wiley \& Sons, Chichester, UK, 2004).

(17)Kim, K.; Reimnitz, L. C.; Cho, S. H.; Noh, J.; Dong, Z.; Gibbs, S. L.; Korgel, B. A. and Milliron, D. J. Effect of nonincorporative cations on the size and shape of indium oxide nanocrystals. Chem. Mater. 2020, 32, 9347-9354.

(18)Ahrén, M.; Selegård, L.; Söderlind, F.; Linares, M.; Kauczor, J.; Norman, P.; Käll, P. and Uvdal, K. A simple polyol-free synthesis route to $\mathrm{Gd}_{2} \mathrm{O}_{3}$ nanoparticles for MRI applications: an experimental and theoretical study. J. Nanopart. Res. 2012, 14, 1006.

(19)Maho, A.; Cabezas, C. A. S.; Meyertons, K. A.; Reimnitz, L. C.; Sahu, S.; Helms, B. A. and Milliron, D. J. Aqueous processing and spray deposition of polymer-wrapped tin-doped indium oxide nanocrystals as electrochromic thin films. Chem. Mater. 2020, 32, 8401-8411.

(20)Leff, D. V.; Brandt, L. and Heath, J. R. Synthesis and characterization of hydrophobic, organically-soluble gold nanocrystals functionalized with primary amines. Langmuir 1996, 12, 4723-4730.

(21)Tan, R.; Yuan, Y.; Nagaoka, Y.; Eggert, D.; Wang, X.; Thota, S.; Guo, P.; Yang, H.; Zhao, J. and Chen, O. Monodisperse hexagonal pyramidal and bipyramidal wurtzite CdSe-CdS core-shell nanocrystals. Chem. Mater. 2017, 29, 4097-4108.

(22)Nagaoka, Y.; Tan, R.; Li, R.; Zhu, H.; Eggert, D.; Wu, Y. A.; Liu, Y.; Wang, Z. and Chen, O. Superstructures generated from truncated tetrahedral quantum dots. Nature 2018, 561, 378-382.

(23)Fabiano, T. A.; Soares, V. P. and Andreoli, E. Pentaethylenehexamine-C60 for temperature consistent carbon capture. $C$ 2015, 1, 16-26.

(24)Zhang, J.; Zhang, H.; Cao, W.; Pang, Z.; Li, J.; Shu, Y.; Zhu, C.; Kong, X.; Wang, L. and Peng, X. Identification of facet-dependent coordination structures of carboxylate ligands on CdSe nanocrystals. J. Am. Chem. Soc. 2019, 141, 15675-15683.

(25)Cass, L. C.; Malicki, M. and Weiss, E. A. The chemical environments of oleate species within samples of oleate-coated PbS quantum dots. Anal. Chem. 2013, 85, 6974-6979.

(26)Barnakov, Y. A.; Idehenre, I. U.; Basun, S. A.; Tyson, T. A. and Evans, D. R. Uncovering the mystery of ferroelectricity in zero dimensional nanoparticles. Nanoscale Adv. 2019, 1, 664-670.

(27)Deacon, G. B. and Phillips, R. J. Relationships between the carbon-oxygen stretching frequencies of carboxylato complexes and the type of carboxylate coordination. Coord. Chem. Rev. 1980, 33, 227-250.

(28)Dong, A.; Ye, X.; Chen, J.; Kang, Y.; Gordon, T.; Kikkawa, J. M. and Murray, C. B. A generalized ligand-exchange strategy enabling sequential surface functionalization of colloidal nanocrystals. J. Am. Chem. Soc. 2011, 133, 998-1006. 
(29)Lutz, H. D.; Himmrich, J. and Schmidt, M. Lattice vibration spectra. Part LXXXVI. Infrared and Raman spectra of baryte-type $\mathrm{TlClO}_{4}, \mathrm{TlBF}_{4}$, and $\mathrm{NH}_{4} \mathrm{BF}_{4}$ single crystals and of ${ }^{11} \mathrm{~B}$-enriched $\mathrm{NH}_{4} \mathrm{BF}_{4}$. J. Alloys Compd. 1996, 241, 1-9.

(30)Ou, Z.; Wang, Z.; Luo, B.; Luijten, E. and Chen, Q. Kinetic pathways of crystallization at the nanoscale. Nat. Mater. 2020, 19, 450-455.

(31)Gao, B.; Arya, G. and Tao, A. R. Self-orienting nanocubes for the assembly of plasmonic nanojunctions. Nat. Nanotechnol. 2012, 7, 433-437.

(32)Israelachvili, J. N. Intermolecular and Surface Forces (Third Edition) Ch. 13, 275 (Academic Press, San Diego, 2011).

(33)La, L. B. T.; Leong, Y. K.; Leatherday, C.; Au, P. I.; Hayward, K. J. and Zhang, L. C. X-ray protection, surface chemistry and rheology of ball-milled submicron $\mathrm{Gd}_{2} \mathrm{O}_{3}$ aqueous suspension. Colloids Surf. A 2016, 501, 75-82.

(34) Salem, L. Attractive forces between long saturated chains at short distances. J. Chem. Phys. 1962, 37, 2100-2113.

(35)Boles, M. A. and Talapin, D. V. Self-assembly of tetrahedral CdSe nanocrystals: effective “patchiness" via anisotropic steric interaction. J. Am. Chem. Soc. 2014, 136, 5868-5871.

(36)Lee, J.; Wang, Z. Y.; Zhang, J. N.; Yan, J. J.; Deng, T. W.; Zhao, Y. Q.; Matyjaszewski, K. and Bockstaller, M. R. Molecular parameters governing the elastic properties of brush particle films. Macromolecules 2020, 53, 1502-1513.

(37)Rubinstein, M. and Colby, R. H. Polymer physics Ch. 3, (Oxford university press, New York, 2003).

(38)Kresse, G. and Joubert, D. From ultrasoft pseudopotentials to the projector augmented-wave method. Phys. Rev. B 1999, 59, 1758-1775.

(39)Kresse, G. and Furthmuller, J. Efficient iterative schemes for ab initio total-energy calculations using a plane-wave basis set. Phys. Rev. B 1996, 54, 11169-11186.

(40)Perdew, J. P.; Burke, K. and Ernzerhof, M. Generalized gradient approximation made simple. Phys. Rev. Lett. 1996, 77, 3865-3868.

(41)Jung, W.; Lee, S.; Yoo, D.; Jeong, S.; Miro, P.; Kuc, A.; Heine, T. and Cheon, J. Colloidal synthesis of single-layer $\mathrm{MSe}_{2}(\mathrm{M}=\mathrm{Mo}, \mathrm{W})$ nanosheets via anisotropic solution-phase growth approach. J. Am. Chem. Soc. 2015, 137, 7266-7269.

(42)Zhang, Z.; Wu, Q.; Johnson, G.; Ye, Y.; Li, X.; Li, N.; Cui, M.; Lee, J. D.; Liu, C.; Zhao, S.; Li, S.; Orlov, A.; Murray, C. B.; Zhang, X.; Gunnoe, T. B.; Su, D. and Zhang, S. Generalized synthetic strategy for transition-metal-doped brookite-phase $\mathrm{TiO}_{2}$ nanorods. J. Am. Chem. Soc. 2019, 141, 16548-16552.

(43)Yang, J.; Zeng, Z.; Kang, J.; Betzler, S.; Czarnik, C.; Zhang, X.; Ophus, C.; Yu, C.; Bustillo, K.; Pan, M.; Qiu, J.; Wang, L. W. and Zheng, H. Formation of two-dimensional transition metal oxide nanosheets with nanoparticles as intermediates. Nat. Mater. 2019, 18, 970-976.

(44) Schneider, C. A.; Rasband, W. S. and Eliceiri, K. W. NIH Image to ImageJ: 25 years of image analysis. Nat. Methods 2012, 9, 671-675. 
(45)Gray, A. T.; Mould, E.; Royall, C. P. and Williams, I. Structural characterisation of polycrystalline colloidal monolayers in the presence of aspherical impurities. J. Phys. Condens. Matter. 2015, 27, 194108.

(46)Frenkel, D. and Smit, B. Understanding molecular simulation: from algorithms to applications Ch. 3, (Elsevier, San Diego, 2001).

(47)Mickel, W.; Kapfer, S. C.; Schroder-Turk, G. E. and Mecke, K. Shortcomings of the bond orientational order parameters for the analysis of disordered particulate matter. J. Chem. Phys. 2013, 138, 044501.

(48)Steinhardt, P. J.; Nelson, D. R. and Ronchetti, M. Bond-orientational order in liquids and glasses. Phys. Rev. B 1983, 28, 784-805.

(49)Zhou, C. C.; Shen, H.; Tong, H.; Xu, N. and Tan, P. Coupling between particle shape and long-range interaction in the high-density regime. Chinese Phys. Lett. 2020, 37, 086301.

(50) Savage, J. R. and Dinsmore, A. D. Experimental evidence for two-step nucleation in colloidal crystallization. Phys. Rev. Lett. 2009, 102, 198302.

(51)Park, J.; Zheng, H.; Lee, W. C.; Geissler, P. L.; Rabani, E. and Alivisatos, A. P. Direct observation of nanoparticle superlattice formation by using liquid cell transmission electron microscopy. ACS Nano 2012, 6, 2078-85.

(52)Zhao, K.; Bruinsma, R. and Mason, T. G. Entropic crystal-crystal transitions of Brownian squares. Proc. Natl. Acad. Sci. 2011, 108, 2684.

(53)Zhao, K.; Bruinsma, R. and Mason, T. G. Local chiral symmetry breaking in triatic liquid crystals. Nat. Commun. 2012, 3, 801.

(54)Hou, Z.; Zhao, K.; Zong, Y. and Mason, T. G. Phase behavior of two-dimensional Brownian systems of corner-rounded hexagons. Phys. Rev. Mater. 2019, 3, 015601.

(55) Anderson, J. A.; Irrgang, M. E. and Glotzer, S. C. Scalable metropolis Monte Carlo for simulation of hard shapes. Comput. Phys. Commun. 2016, 204, 21-30.

(56)Anderson, J. A.; Lorenz, C. D. and Travesset, A. General purpose molecular dynamics simulations fully implemented on graphics processing units. J. Comput. Phys. 2008, 227, 53425359.

(57) Schapotschnikow, P.; Pool, R. and Vlugt, T. J. Molecular simulations of interacting nanocrystals. Nano Lett. 2008, 8, 2930-2934.

(58)Binder, K. and Milchev, A. Polymer brushes on flat and curved surfaces: How computer simulations can help to test theories and to interpret experiments. J. Polym. Sci., Part B: Polym. Phys. 2012, 50, 1515-1555.

(59)Widmer-Cooper, A. and Geissler, P. L. Ligand-mediated interactions between nanoscale surfaces depend sensitively and nonlinearly on temperature, facet dimensions, and ligand coverage. ACS Nano 2016, 10, 1877-1887.

(60)Gao, H.; Bettscheider, S.; Kraus, T. and Muser, M. H. Entropy can bundle nanowires in good solvents. Nano Lett. 2019, 19, 6993-6999. 
(61) Sanchez-Iglesias, A.; Grzelczak, M.; Altantzis, T.; Goris, B.; Perez-Juste, J.; Bals, S.; Van Tendeloo, G.; Donaldson, S. H., Jr.; Chmelka, B. F.; Israelachvili, J. N. and Liz-Marzan, L. M. Hydrophobic interactions modulate self-assembly of nanoparticles. ACS Nano 2012, 6, 1105911065.

(62)White, L. R. On the Deryaguin approximation for the interaction of macrobodies. J. Colloid. Interf. Sci. 1983, 95, 286-288.

(63) Johnston, K. and Harmandaris, V. Properties of short polystyrene chains confined between two gold surfaces through a combined density functional theory and classical molecular dynamics approach. Soft Matter 2012, 8, 6320-6332.

(64)Bussi, G.; Donadio, D. and Parrinello, M. Canonical sampling through velocity rescaling. J. Chem. Phys. 2007, 126, 014101.

(65)Irshad, K. A.; Anees, P.; Rajitha, R.; Ravindran, T. R.; Srihari, V.; Kalavathi, S. and Shekar, N. V. C. Anomalous lattice compression in the hexagonal $\mathrm{La}_{2} \mathrm{O}_{3}-\mathrm{A}$ high pressure X-ray diffraction, Raman spectroscopy and first principle study. J. Alloys Compd. 2020, 822, 153657.

(66)Jiang, S.; Liu, J.; Bai, L. G.; Li, X. D.; Li, Y. C.; He, S. M.; Yan, S. and Liang, D. X. Anomalous compression behaviour in $\mathrm{Nd}_{2} \mathrm{O}_{3}$ studied by $\mathrm{X}$-ray diffraction and Raman spectroscopy. AIP Adv. 2018, 8, 025019.

(67)Lonappan, D.; Shekar, N. V. C.; Sahu, P. C.; Kumarasamy, B. V.; Bandyopadhyay, A. K. and Rajagopalan, M. Cubic to hexagonal structural transformation in $\mathrm{Gd}_{2} \mathrm{O}_{3}$ at high pressure. Philos. Mag. Lett. 2008, 88, 473-479. 